\title{
PROCEEDINGS OF THE FORTY-FOURTH ANNUAL MEETING OF THE AMERICAN SOCIETY FOR CLINICAL INVESTIGATION HELD IN ATLANTIC CITY, N. J., MAY 5, 1952
}

\author{
Presidential Address
}

THE "LOGARITHMIC PHASE" OF MEDICAL PROGRESS

BY W. BARRY WOOD, JR.

By custom the presiding officer of this society is expected to open the scientific session with a presidential address. This obligation, which I accept with grave misgivings, I shall try to fulfill in as short a time as possible, so that we may proceed promptly to the main program.

The thesis which I should like to present briefly, is that today we find ourselves in, or rapidly approaching, the "logarithmic phase" of medical progress.

The choice of my crude analogy is, of course, conditioned by my own past training and interests. Reference to the bacterial growth curve may be justified, however, on the grounds that it is familiar to nearly everyone in medicine (Figure 1). You will recall that the logarithm

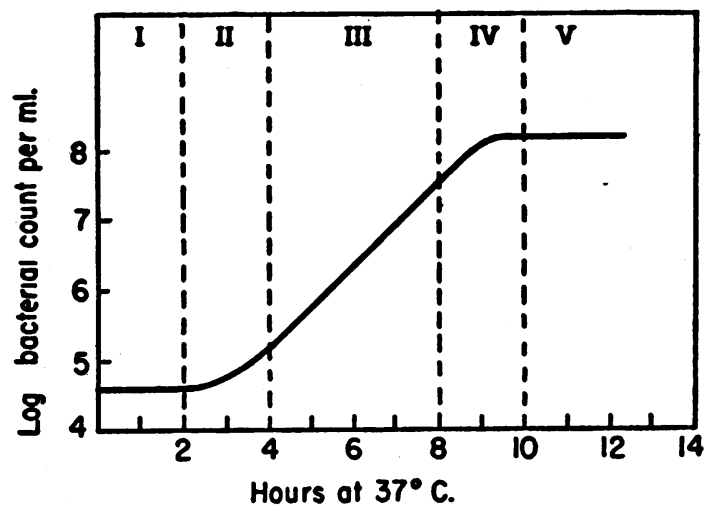

Fig. 1. Conventionalized Curve of Bacterial Growth

of the number of viable organisms in a bacterial culture is customarily plotted on the vertical axis and the duration of incubation (in hours) on the horizontal. When cultural conditions are optimal, the curve exhibits five more or less separate phases of growth.

The first phase, usually referred to as the lag phase, is characterized by a stationary population. To consider it a latent period in the growth cycle is, as I shall explain in a moment, incorrect. The second period represents the transition from a stable population to one of rapid multiplication. The relatively gradual acceleration of multiplication in this transitional period is attributed to the failure of all of the cells to start multiplying at exactly the same moment. During the third phase of growth, the familiar logarithmic phose, all viable cells are presumably dividing at a maximum rate, causing the population of the culture to increase exponentially. Phases
IV and V represent the final deceleration of growth, ending in a relatively stable population.

Today we need not consider the last two phases of the curve except to recall that premature cessation of bacterial growth may be caused by adverse cultural conditions, as well as by the addition of antimicrobial agents which interfere with the metabolism of dividing cells. The analogy here is obvious to anyone who has contemplated the possible effects on medical science of atomic warfare, of a sudden collapse or further disorganization of world economy, of an emphasis upon quantity instead of quality in medical education, of a sudden drying up of sources of research funds, or of a requirement for "responsible investigators" to prepare more progress reports than we are already required to do. Any one of these and a host of other unpleasant eventualities might cause a significant deceleration of medical progress even within our own lifetimes.

I should like to suggest that we turn our backs on these disturbing thoughts and return for a moment to the past, or, in other words, to the first phase of the growth curve. As I have already stated, the lag phase is not in reality a latent period. On the contrary, there is good evidence that individual cells in bacterial cultures are metabolically more active late in the lag phase than in any other phase of the cycle. This accelerated metabolism, unassociated with rapid cell division, causes two significant changes to take place in the culture. First, it results in an increased protoplasmic mass of individual cells; the oversized or giant organisms of the late lag phase are thus formed (Figure 2). Secondly, it brings about important alterations in the chemical environment of each organism-changes essential to the process of multiplication.

The counterparts of these two characteristics of the lag phase are not difficult to find in the early history of medicine. Viewed from our present vantage point on the steeply sloping curve of modern achievement, the era from Hippocrates to Claude Bernard may appear to have been a latent period, but, like the lag phase of bacterial growth, it teemed with ferment, particularly during its later decades. This ferment likewise gave rise to giantsHarvey, Leeuwenhoek, John Hunter, Lavoisier, and Liebig, to mention but a few. Like the oversized bacterial cells of the lag phase, these great figures of the past exerted a profound influence upon their environments, an influence which paved the way for the "logarithmic 
phase" of scientific progress, in which we now find ourselves.

The century of medicine between Claude Bernard and the present was one of rapid acceleration, quite analogous to the steepening slope of phase II of the growth curve. Just as the oversized bacteria of the lag phase are replaced by the smaller rapidly dividing cells which characterize the logarithmic phase, so have the medical giants of the late nineteenth and early twentieth centuries given way to a host of well-trained scientists, educators, and physicians, individually of lesser stature but collectively of far greater potential. As the standards of medicine have risen, the prominence of the few has become relatively dwarfed by the accomplishments of the many.

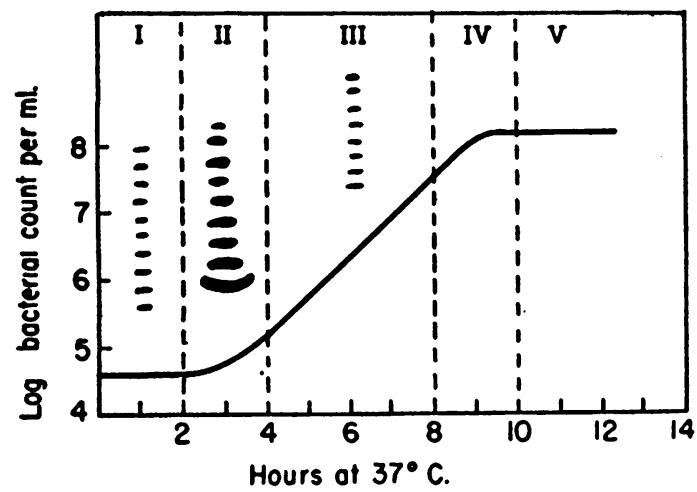

Fig. 2. Diagram Indicating Changes that Occur in the Size of the Bacterial Cells during the First Three Phases of the Growth Curve

Whether or not, in 1952, we have truly reached the "logarithmic phase" of medical progress may certainly be debated. It may be argued that progress will continue to accelerate for years or even decades before it enters an exponential phase. No one can deny, however, that the curve upon which we now find ourselves at the mid-century has a far steeper slope than that along which the founders of this society were moving more than forty years ago. The increase in slope has brought profound changes upon literally every phase of medicine. We would do well to examine briefly a few of their more striking effects upon us as physicians, teachers, and investigators.

First as physicians, engaged in the care of ill patients, we are keenly aware of our inability to keep abreast of even the most important advances in clinical medicine. When we scan the contents of medical journals, we are appalled by the volume of new information with which we should be familiar. To meet this problem, which every day is becoming more difficult, we have had only one recourse-namely, to specialize. No matter how much the trend toward specialization is decried by the Academy of General Practice, by medical economists, by educators, or by the lay public, it is here to stay, for it is an inevitable result of the steepness of the progress curve. The era when a single doctor could be an ex- pert in all of medicine, or even in all of internal medicine, has long since passed. To bring to his patients the best that medicine has to offer, the physician of today must limit the scope of his practice to that field in which he is able to remain competently trained.1 If his acquisition of new knowledge ends with his completion of formal schooling or his receipt of a certificate from a specialty board, he is doomed to an ignominious fate of professional obsolescence.

Secondly, the accelerated tempo of medical progress has affected medical education fully as much as it has influenced clinical practice. Only too well does the present generation of students and teachers realize that what is taught today may be outmoded tomorrow. The steepening curve of progress not only causes textbooks to become obsolete with discouraging promptness, but also renders senior faculty members prematurely senile in the eyes of their younger colleagues. Refined laboratory methods have increased the accuracy of present day diagnoses far beyond the dreams of the most skillful elders of the past. These precise diagnostic tools, as well as the numerous specific agents of modern therapy, require for their proper use a more intimate knowledge of chemistry, physics, and pathologic physiology than was ever before considered relevant to clinical medicine. So vast has this body of pertinent preclinical information become that no one teacher of medicine can possibly master it all. To meet the situation, he, like the practitioner, has been forced to resort to specialization.

Let us contrast for a moment a department of medicine of today with one of four or five decades ago. At the turn of the century teaching services were usually composed of a single professor, a resident, and a handful of house officers. The professor was met ceremoniously each morning at the front door by his resident and escorted to the wards for the daily round. There, followed by an attentive retinue of students and house officers, he passed final judgment on every manner of problem encountered at the bedside. Because of his vast experience in the management of patients, his opinions were rarely questioned, and his orders were followed to the letter.

How, I wonder, would such a versatile soloist of the clinic have viewed his counterpart of the mid-twentieth century? The modern professor of medicine, whatever else he may be, is certainly no soloist. To meet the requirements of progressive teaching, he has gathered about him a galaxy of highly trained colleagues, whose combined knowledge he hopes will cover the rapidly expanding fields of internal medicine. No longer a virtuoso, he has become the conductor of an orchestra composed of experts in an ever-increasing number of sub-

1 By this statement I do not imply that internists should practice only subspecialties. Quite to the contrary, I believe that every physician should be broadly trained and should strive to maintain his competence in as much of general medicine as possible. At the same time, aware of his deficiencies, he should seek the aid of an expert consultant whenever his own ignorance may jeopardize the welfare of a patient. 
specialties. Weekly at grand rounds, before an audience of students, house officers, and staff, he is expected to conduct a concert in which he displays the talents of his various experts by allowing them in turn to carry the melody. For any discord that results he is held personally responsible. In some respects, his assignment might frighten even Toscanini, for just as the orchestra is trained to perfection, one of its talented members will suddenly introduce a new instrument-a longer, more versatile catheter, an artificial kidney, another triumph of chemotherapy. With each innovation the professor is expected to become quickly familiar, for as conductor he must realize its possibilities and limitations, in order to integrate it with the rest of the orchestra. Judging from my own brief experience in this exacting role, I wonder if the average professor of medicine today ever really "knows the score."

Finally, the character of medical research has been changed by the rapidity of scientific advance. As the body of knowledge available to medical investigators has increased, so has the need for specialization in research become mandatory. Team projects are the fashion of the day. To be sure, many problems can be solved only by collaboration of experts from a variety of fields. But as every scientist knows, ideas come from individuals, not from groups or institutes. Again the giants of the re- cent past-Claude Bernard, Pasteur, Emil Fischer, and Ehrlich, each of whose contributions to medical science cover a wide range of subjects-have been succeeded by men whose research has perforce been limited to a lesser scope. Today, more than ever before, individual workers must concentrate their efforts on a single, relatively narrow field, in order to contribute significantly to medical knowledge. To dig deeply at the frontiers of modern medicine requires decades rather than days, the whole life of the investigator rather than the term of his research grant.

To its medical scientists, contemporary society has been most generous. Well has it heeded the advice of Pasteur: "Take interest, I implore you, in those sacred dwellings which one designates by the expressive term, laboratories. Demand that they be multiplied, that they be adorned. These are the temples of the future."

The scientific program, which we are about to hear, and the large number of equally excellent reports which I was forced to omit from the final program, provide convincing evidence that the clinical investigator of today, in spite of daily distractions of practice, teaching, and administration, is making good use of his laboratories. The variety and content of the individual papers will, I am sure, eloquently support the general thesis that the slope of the medical progress curve as of 1952 is indeed steep. 


\section{ABSTRACTS}

Body Potassium Deficiency in Diabetes Mellitus. J. K. Aikawa, J. H. Felts and G. T. Harrell, JR,* Winston-Salem, N. C.

The body content of exchangeable potassium $\left(\mathrm{K}_{\bullet}\right)$ was measured by the radioisotope $\left(\mathrm{K}^{\circ}\right)$ dilution technique in 42 diabetic patients, 22 females and 20 males, age range -18 to 78 years. Patients were hospitalized for regulation, for treatment of complications, or for therapy of unrelated diseases. The diabetes varied widely in severity, duration and adequacy of control; 2 patients were in acidosis and coma. All but one received insulin.

The mean initial $K_{e}$ value in the females was $27.3 \pm$ s.d. $9.77 \mathrm{meq} . / \mathrm{kg}$., with a range between 12.9 and 50.5 meq./ kg.; the normal value in young females is $31.5 \mathrm{meq} . / \mathrm{kg}$. In 20 males, the mean was $31.4 \pm$ s.d. 7.25 meq. $/ \mathrm{kg}$., with a range between 16.6 and $53.4 \mathrm{meq} . / \mathrm{kg}$., compared with a normal of $46.3 \mathrm{meq} . / \mathrm{kg}$.

In 20 subjects, fifty serial determinations were performed, at weekly intervals when possible, in an attempt to determine whether the $K_{0}$. would change. In 15 instances, following the initial determination, an oral supplement of $\mathrm{KCl}, 3 \mathrm{gm}$. daily, was given; 6 of these retained potassium. Two of 5 patients not given a supplement retained dietary potassium as control of the diabetes improved or as other disease processes were corrected. No change was noted in $\mathrm{K}_{\text {. in the remaining }}$ 12 patients. All serum potassium values throughout the study were within normal limits $(3.5$ to 5.0 meq. $/ \mathrm{kg}$.) as determined by flame photometry. It was not always possible to predict from the initial $\mathrm{K}_{\text {. }}$ value, the state of regulation, or the intercurrent disease whether the patient would retain potassium.

These data suggest that a body potassium deficit may exist, not only in patients with diabetic acidosis and coma, but also in those diabetics without clinical, chemical or electrocardiographic evidence of such a deficiency.

Triethylene Melamine in Malignant Disease. Lours $\mathrm{K}$. AlPERT, Washington, D. C. (Introduced by Theodore J. Abernethy).

Fifty-two patients with various malignant diseases, predominantly lymphomas, were treated with Triethylene melamine (TEM) by oral administration. In approximately two-thirds of the patients with lymphomatous disorders, the response was good or excellent, as manifested by a major degree of regression of lymph node masses or return of hematologic status toward normal, with pronounced symptomatic improvement. The patients with lymphosarcoma or chronic lymphatic leukemia were more readily controlled than those with Hodgkin's disease or chronic myeloid leukemia. Those who failed to improve were in the late stages of their disease when therapy was started.

* Member.
In the acute leukemias, the number of circulating white cells was reduced, but the toxic features of the disease were not altered, and in some instances it appeared that TEM may have had a deleterious effect. No objective improvement was noted in the patients with fibrosarcomas or carcinomas of the lung, stomach, thyroid or colon.

The sensitivity of the lymphomas to TEM in the individual patients was unpredictable, as indicated by the wide variation in the total dosage, of from 5 to $200 \mathrm{mgm}$., required to produce a maximum effect. Hence, the therapy could not be standardized, but was determined by an intermittent and gradually increasing schedule, over periods ranging from one to twenty-three weeks.

Hematopoietic depression was the limiting factor in the total amount of TEM which could be administered, particularly to patients with a high degree of "activity" of their disease.

The demonstrated effectiveness of Triethylene melamine in the therapy of lymphomas brings it within the current concept of the management of malignant disease by agents which will permit the temporary return of a significant percentage of patients to a normal manner of living, even though permanent eradication of the disease is not produced.

Study of Left Atrial Pressure Waves in Relation to Pulmonary "Capillary" Pressure Waves in Patients with Mitral Stenosis. William H. Ames, Ernest 0. Theilen, JohanN L. Ehrenhaft and James W. Culbertson, Iowa City, Iowa. (Introduced by William B. Bean).

Left atrial pressure waves have been recorded at operation for mitral valvuloplasty in seven patients by means of a catheter inserted through the auricular appendage and connected to an electromanometer. Pulmonary "capillary" pressure waves likewise have been registered preoperatively in ten patients. These two types of wave forms were available for direct comparison in four of the patients. There was good positive correlation between left atrial and pulmonary "capillary" waves with respect to both pressure levels and wave contours. Left atrial mean pressures were equal to or greater than the pulmonary "capillary" mean pressures. Patients with normal sinus rhythm exhibited two distinct waves in each location, the first following the onset of electrical systole of the atrium and the second following the onset of electrical systole of the ventricle. Analysis of this second wave yielded clinically useful information concerning the degree of mitral regurgitation, even in the presence of auricular fibrillation.

Planimetric measurements of corresponding left atrial and pulmonary "capillary" regurgitant pulse wave areas were in close agreement. In the four patients the left atrial waves varied considerably, with mean values of $\mathbf{5 . 2}$ 
to $9.4 \mathrm{~mm} \mathrm{Hg}$, and pulmonary "capillary" waves were proportionately lower (3.3 to 7.9 ). These values must be distinguished from the mean left atrial and pulmonary "capillary" blood pressure levels, which ranged from 24 to 34 and 17 to $24 \mathrm{~mm} \mathrm{Hg}$ respectively. All records were analyzed thus and the results found to agree with clinical impressions made by physical examination previously and by intra-atrial palpation at operation.

These observations indicate that pulmonary "capillary" pressure recording may provide helpful information for the evaluation of patients prior to mitral valvuloplasty and for the assessment of postoperative results by objective physiological methods.

\section{Minor Respiratory Diseases: Studies of $M R-3$ Virus Disease in Human Volunteers. LEON T. ATLAs, Bos- ton, Massachusetts. (Introduced by John G. Gibson 2nd).}

Nasal washings were collected from a donor early after onset of respiratory symptoms. His nasal washings and nasal washings from volunteers infected by his secretions were inoculated intra-nasally into 133 volunteers kept in isolation. Third to fourth passage allantoic fluids of embryonated eggs inoculated with the donor's nasal washings were instilled into 47 volunteers.

It was found that both nasal washings and egg cultivated virus produced a consistent and specific disease entity in volunteers, whose illnesses recapitulated the signs and symptoms suffered by the donor. Volunteers receiving normal control inocula had no such disease. The signs and symptoms responsible for the observers' subjective judgment of severity of disease or no disease were determined by statistical analysis of the recorded data. Following a three to five day incubation time, two out of three of running nose, coughing, and chest pain developed in all men considered as definitely sick. A profuse rhinitis was observed in all moderate or severe infections. In addition, conjunctivitis, headache, malaise, muscle and joint aches, fever, and leucopenia were often present. Some volunteers developed lingering weakness, persistent coughing and chest pains resembling those of acute benign pericarditis.

Infection by $M R-3$ virus produced a significant resistance to homologous reinfection for 8 months. However, 9 of 22 men upon homologous reinoculation developed an atypical disease characterized by minimal upper respiratory signs but one or more of malaise, fever, coughing, chest pain and migratory polyarthralgia. Recovery from $M R-3$ infection did not prevent infection by $M R-1$ or $M R-4$ viruses. Transmission of $M R-3$ disease from infected to contact volunteers was demonstrated. Cultivated fluids beyond the fifth egg passage produced disease so attenuated in incidence and severity that objective evaluation of clinical findings was no longer practical.

The Relation of Colonial Morphology and Antigenic Structure to Virulence in Pneumococcus. ROBERT Austrian, Baltimore, Md. (Introduced by Palmer $\mathbf{H}$. Futcher).
Attempts to relate colonial morphology, antigenic structure and virulence in a variety of bacterial species have resulted, at times, in apparent inconsistencies. A study of these bacterial properties in pneumococcus employing selective cultural and serologic techniques and transformation reactions has afforded a better understanding of their interrelationships.

With the definition of the rough encapsulated variant in the experiments reported here, four distinct colonial forms of pneumococcus may now be recognized: smooth encapsulated, rough encapsulated, smooth unencapsulated, and rough unencapsulated. A difference between the smooth and rough variants appears to reside in the mode of separation of their cells after division. Cells of smooth variants separate into pairs or short chains after division whereas those of rough forms remain attached forming chains of great length. This characteristic of rough variants is responsible apparently for the irregular surfaces and margins of their colonies on solid media and is manifested both by encapsulated and unencapsulated forms.

Antigenic analysis of smooth and of rough unencapsulated pneumococci reveals both to possess species-specific C carbohydrate and type-specific $\mathbf{M}$ protein but both are phagocyted readily by leukocytes and lack virulence for mice. Encapsulated smooth and rough organisms produce, in addition to the antigens mentioned, type-specific capsular polysaccharide, are not phagocyted readily in the absence of homologous anticapsular antibody and are virulent for mice.

Studies of colonial variation in pneumococcal transformation reactions demonstrate that the genetic determinants controlling encapsulation and the mode of cell separation are inherited independently of one another. These experiments show clearly that the principal factor in the virulence of pneumococcus is the presence or absence of encapsulation regardless of whether the mode of cell separation after division is that of the rough or that of the smooth variant.

Urinary Excretion of Tetrahydrocortisone. BILLY BAGGETT, JoHN H. GLICK, JR. and RALPh A. Kinsella, JR., St. Louis, Mo. (Introduced by Ralph Kinsella).

Tetrahydrocortisone (pregnane-3 $\alpha, 17 \alpha, 21$-triol-11,20,dione) has been identified as the principal $\alpha$-ketolic corticoid in the urine of normal adults and healthy pregnant women. Neutral reducing lipids and neutral formaldehydogenic lipids can be extracted in greatly increased amounts following hydrolysis of their urinary conjugates with a bacterial enzyme preparation containing $\beta$-glucuronidase, as previously reported. This hydrolysis results in ten to fiftyfold increases in extractable tetrahydrocortisone, in contrast to the increases in extractable Compounds $E$ and $F$ which are three to fourfold. Quantitative estimations of urinary tetrahydrocortisone and Compounds $\mathrm{E}$ and $\mathrm{F}$ have been made after separation by paper chromatography. Tetrahydrocortisone has been isolated as the pure crystalline compound and identified; the acetate was prepared for further identification. Urinary excretion of tetrahydrocortisone has been found in normal adults to be 
from 3 to $6 \mathrm{mg}$ in 24 hours and in pregnant women to be slightly lower than the lowest values obtained for normal adults. Studies are now in progress to determine the relationship between urinary excretion of tetrahydrocortisone and the administration of ACTH and adrenal steroids.

Acute Responses of $T m_{P A B}$ and Glomerular Filtration Rate to Intravenous Lactate in Uremic Patients. EARL S. Barker, John Kapp Clark * and Archer P. Crosley, Jr., Philadelphia, Pa.

The recent demonstration of acute increases in $T m_{P A B}$ and glomerular filtration rate (GFR) following intravenous administration of hypertonic sodium lactate in normal subjects might indicate the mechanism of the clinical improvement in uremia said to result from chronic lactate administration. These measurements were therefore made directly in patients with azotemia due to various renal diseases.

Increases in $\operatorname{Tm}_{\mathbf{P A B}}$, inulin clearance and endogenous creatinine clearance following lactate 6 in severe uremics were negligible considered in absolute terms (i.e. increases of less than $3 \mathrm{mgm} . / \mathrm{min}$. in $T \mathrm{~m}_{\mathrm{PAB}}$ and $4 \mathrm{cc} . / \mathrm{min}$. in GFR). However, in terms of percentage increase, the changes following lactate were of the same order of magnitude in uremics of different degrees and in normals (about $30 \%$ in $\mathrm{Tm}_{\mathrm{PAB}}$ and somewhat less in GFR).

These data indicate that, so far as tubular transport mechanisms involved in $\mathrm{PAH}$ excretion are concerned, each unit of remaining function is capable of responding normally and suggest that even severe renal insufficiency in several types of renal disease may be associated with quantitative reduction rather than qualitative change in the particular system tested. Whatever benefit may be derived from lactate treatment by these mechanisms must accrue from very small changes in absolute terms. Nevertheless it is believed that further study is warranted, since these patients are surviving on extremely small margins of renal function and the changes may therefore be clinically important.

Biochemical Abnormalities in Wilson's Disease. A. G. Bearn and Henry G. Kunkel,* New York, N. Y.

In view of recent reports describing changes in copper metabolism in Wilson's disease, a special study of 9 patients suffering from this condition was undertaken. Despite wide differences in age, duration of the disease and degree of hepatic and neurological involvement, all patients showed a marked increase in the daily urinary excretion of copper (range 450-1790 gamma/24 hoursnormal 0-25 gamma/24 hours). As a corollary to this study, quantitative analyses for 19 amino acids in the urine from 6 of these patients were carried out by Drs. William H. Stein and Stanford Moore using ion exchange columns. In 5 of these cases the excretion of tremendously increased quantities of 13 of the amino acids was observed (up to or more than 30 times normal, in some instances). Ornithine and citrulline, two amino acids not encountered in normal urine, were present in significant amounts. A striking parallelism was found between the copper and amino acid excretion which suggests that a metabolic relationship may exist between these two defects.

Analyses of serum copper showed that the levels were reduced in 8 of the 9 patients (39-79 gamma/100 mlnormal 90-120 gamma/100 ml), a somewhat surprising finding in view of the increased urinary excretion of this metal. Copper is known to be the prosthetic group of certain enzymes and in serum is present largely as an oxidase. A considerable decrease in serum oxidase activity was observed in all patients thus correlating with the low copper levels. The administration of B.A.L. resulted in an increase in the urinary copper excretion in all patients to whom it was given, and in some instances was followed by slight clinical improvement.

\section{Clinical and Metabolic Effects of Bilateral Adrenalectomy}

in Man. D. M. Bergenstal and C. B. Huggins, Chicago, Illinois. (Introduced by A. T. Kenyon).

Bilateral simultaneous adrenalectomy has been carried out by us on 35 human cases with two deaths. An adequate hormonal substitution program has been developed to prevent adrenal insufficiency, and in most cases adequate maintenance hormonal substitution has been $25 \mathrm{mg}$. cortisone twice a day by mouth and three grams of sodium chloride. Patients with metastatic cancer were adrenalectomized. In 7 cases of prostatic cancer which became reactivated after previous antiandrogenic control, adrenalectomy gave relief of bone pain, increased appetite with weight gain, reduction in acid phosphatase when elevated, elevation of alkaline phosphatase, and in many cases produced shrinkage of tumor. There have been two relapses. Cortisone and ACTH did not give sustained beneficial effect in prostatic cancer. Five cases of mammary cancer have shown significant remission characterized by either regression of tumor, decrease in hemothorax or healing of bone lesions. The miscellaneous group of tumors showed no significant alteration in their course after adrenalectomy. It is reasonable to assume that the regressions are due in part to elimination of critical amounts of sex hormones by adrenalectomy.

The eosinophile test was negative after adrenalectomy in response to saline and $\mathrm{ACTH}$, but a greater than $\mathbf{5 0}$ per cent decrease was observed in 6 out of 9 patients given adrenalin. The glucose tolerance, insulin tolerance and Kepler water tests were not significantly altered after operation. A study of 17-ketosteroids and dehydroisoandrosterone excretion before and after adrenalectomy has been done. Alterations in excretion of these steroids (in patients without gonads and adrenals) during episodes of stress and after administration of compounds B, E, F and dehydroisoandrosterone have been studied. Three cases of hypertension, as well as the normotensive patients, have stabilized their blood pressures near the pre-operative levels. Two patients with diabetes mellitus showed no change in insulin requirement after adrenalectomy. 
Bone as a Sodium and Potassium Reservoir. William H. Bergstrom, Boston, Mass. (Introduced by James L. Gamble).

Bone contains a large fraction (approximately 25\%) of the total amount of sodium present in the body. This report describes an investigation of bone sodium and potassium content and of the availability of these cations during acute electrolyte depletion.

We have determined sodium, potassium and chloride in the bones of groups of normal rats at several stages of maturity. Average sodium concentrations, corrected for extracellular fluid, ranged from $110 \mathrm{mEq} / \mathrm{Kg}$ in young rats to $180 \mathrm{mEq} / \mathrm{Kg}$ in adult rats. Potassium showed an inverse relation to age, falling from $35 \mathrm{mEq} / \mathrm{Kg}$ in immature rats to $19 \mathrm{mEq} / \mathrm{Kg}$ in mature rats. These values were corrected for potassium calculated to be present in marrow.

Base depletion was accomplished by intraperitoneal dialysis. The procedure resulted in an average loss of $12 \mathrm{mEq}$ of sodium per $\mathrm{Kg}$ of body weight. Mature and immature rats sacrificed 48 hours after depletion showed bone sodium values averaging $25 \mathrm{mEq} / \mathrm{Kg}$ less than those of the appropriate age controls. Bone potassium was $12 \mathrm{mEq} / \mathrm{Kg}$ below its control concentration.

The data indicate that bone acts as a reservoir from which sodium and potassium are drawn during acute base depletion. The magnitude of the changes observed may be illustrated by reference to a $70 \mathrm{Kg}$ man, in whom comparable values would imply a contribution of 300 $\mathrm{mEq}$ of sodium and $150 \mathrm{mEq}$ of potassium to the body base economy, assuming the skeleton to comprise $17 \%$ of body weight. The relation of these findings to the interpretation of metabolic balance studies will be discussed.

The Distribution and Utilization of Radio-Iron $\left(F e^{50}\right)$ in Protein-Depleted Rats. W. F. BETHARD, R. W. WISsLER, J. S. Thompson and M. J. Robson, Chicago, Illinois. (Introduced by L. O. Jacobson).

Protein deprivation in young, adult, male rats was followed promptly by marked diminution of hemoglobin formation, as measured by incorporation of radio-iron into erythrocytes. Within two weeks only $4.4 \%$ of the intravenous tracer appeared in erythrocytes of proteindepleted animals, as compared to $59.1 \%$ in the erythrocytes of controls. Diminished hemoglobin production was sustained for 35 days, after which protein repletion was instituted. Addition of protein to the diet resulted in rapid restoration of hemoglobin formation to normal. The rapidity and degree of alterations in hemoglobin formation could not be satisfactorily quantitated by either peripheral blood counts or blood volume determinations.

At the nadir of protein depletion, radio-iron tissue distribution was determined. The usual chronology of iron metabolism was completely disrupted. Protein-depleted livers of the experimental group accumulated an average of $46.4 \%$ of injected tracer, despite the fact that iron is presumably stored as protein conjugate. Bone marrow of this group contained only $5.59 \%$ of the radio-iron at 3 hours; and a maximum of $33.6 \%$ appeared at 36 hours.
In contrast, control animals yielded $61 \%$ of the tracer in the bone marrow at 3 hours, after which an exponential disappearance occurred. Normal livers consistently contained $11 \%$ of the injected dose. In tissues examined, $78.6 \%$ of the radio-iron was recovered in control animals, but only $53.8 \%$ could be accounted for in proteindepleted rats.

New quantitative and temporal alterations in hemoglobin formation during protein depletion have been shown using radio-iron tracer techniques. Disruption of normal time-concentration tissue iron relationships has similarly been demonstrated. These results may help explain the anemia associated with chronic infections, malignant neoplasms, and other debilitating diseases.

The Chemical Determination of Lipids in Needle Biopsy Specimens of Liver. Barbara H. Billing, David E. Hein and Harold J. Conlon, Cincinnati, Ohio. (Introduced by Leon Schiff).

In the past, estimations of the lipid content of the human liver have been confined to autopsy material. With the development of needle biopsy techniques, liver lipids can now be determined chemically during life. The biopsy specimen (3-8 mg.) is transferred from the needle to a hook and is immediately weighed with a quartz fibre balance at 15 second intervals for 3 minutes so that a correction for water lost by evaporation can be made. The specimen is frozen, dried in a vacuum desiccator, reweighed and then placed in a tube containing $500 \mu l$ ethyl alcohol. The tube is sealed and after 48 hours at $60^{\circ} \mathrm{C}$, the specimen is dried and reweighed; the amount of fat extracted can then be calculated. Aliquots of the alcohol extract are used for the determination of phospholipids, total fatty acids and iodine number.

In order to show that tissue obtained by needle biopsy gives a valid representation of the whole liver, in respect to lipid content, needle specimens have been obtained from 18 sites in each of 20 livers obtained at autopsy. Values for the lipid content of the specimens showed good agreement and correlated well with the concentration in the entire liver. In contrast to observations made on experimental animals, the quantitative determinations indicated a relatively uniform distribution of lipids in the liver. Comparison of the histological and chemical findings has made it possible to assign quantitative values to the microscopic grading of fat in the liver.

Forty-six routine liver biopsies taken from 40 patients for diagnostic purposes have been analysed chemically as well as microscopically. No correlation between the concentration of lipids in the blood and the liver has been found.

The Effect of Expansion of the Extracellular Fluid Volume in Conditioning the Diuretic Response to Ingested Isotonic Saline Solution. William H. BIRCHard, Framingham, Massachusetts. (Introduced by Maurice B. Strauss).

The long-recognized fact that man exhibits little increase in urine flow following the ingestion of isotonic 
saline solution has been considered evidence of a lack of responsiveness to changes in the volume of extracellular fluid.

However, it has been shown that expansion of extracellular fluid volume by the relatively rapid intravenous infusion of isotonic saline produces a water diuresis in the recumbent subject. The following experiments indicate that the ingestion of isotonic saline also produces a diuretic response when there has been prior expansion of the extracellular fluid volume.

Three normal subjects, each seated quietly in a chair, several hours after breakfast, ingested 1 liter of $0.9 \%$ sodium chloride solution. During the ensuing three hours urine flow remained below $1 \mathrm{ml} . / \mathrm{min}$., and urinary electrolyte concentration rose to 600 milli-osmols $/ 1$.

Subsequently, but before 6 P.M., each subject ingested sufficient saline to constitute a load of $6.2 \mathrm{meq} . / \mathrm{kg}$. in addition to salt taken with food.

On the following morning weight gains averaged 0.8 kg., ascribable to an expansion of extracellular fluid volume. The subjects again ingested 1 liter of $0.9 \%$ saline. There now occurred prompt diureses of approximately one hour's duration averaging $7.5 \mathrm{ml} . / \mathrm{min}$., with urine specific gravities falling to 1005 and urinary electrolyte concentrations to 140 milli-osmols/1.

Thus it would appear that the ingestion of salt solution by subjects whose extracellular fluid volume already is expanded produces a type of diuresis characteristic of decreased antidiuretic activity.

Neural Control of Renal Excretion of Sodium in the Dog. Wirliam D. Blake, New Haven, Conn. (Introduced by Dr. C. N. H. Long).

Recently Kaplan and associates have reiterated the viewpoint that renal denervation increases electrolyte excretion independent of increased filtration rate, primarily on the basis of maintaining high solute concentration in the urine. The present studies confirm the main theses that increased filtration rate is unnecessary for increased sodium output on the denervated side, that the effect is most apparent in the fasting or hydropenic animal and that the significant difference between denervated and control kidneys is the much higher concentration of sodium in the urine from the denervated kidney. The intrarenal mechanisms responsible for this difference in concentration are not clear. With this in mind an attempt has been made to develop a pharmacological technique for studying neural control of renal function. Studies have been done on dogs, with and without pentobarbital anesthesia, under varying conditions of water and salt balance. The usual clearance techniques were used to estimate renal function. Drugs used were adrenalin or synthetic epinephrine and hydergine (Sandoz), a combination of dihydrogenated ergot alkaloids. Studies in this laboratory and elsewhere indicate that epinephrine acts directly on the kidney and may well be the mediator of neural impulses. Conversely, hydergine appears to act, in part at least, by blocking nervous stimuli (data to be presented elsewhere). In hydropenic, unanesthetized dogs infusion of small amounts of adrenalin leads to a marked drop in urinary sodium concentration, whereas the administration of hydergine usually has the opposite effect, i.e. sodium concentration increases. These effects are not noted in dogs made hypervolemic by the infusion of "normal" saline. Beside hydropenia neural control of electrolyte excretion may be a factor in emotional stress and pentobarbital anesthesia.

\section{Bone Marrow Studies in Polycythemia. Matrhew} Block and William F. Bethard, Chicago, Illinois. (Introduced by Clayton Loosli).

In 42 of 47 cases of polycythemia vera, including patients with normal or low hemoglobin, hematocrit and erythrocyte values following phlebotomy, phenylhydrazine or spontaneous hemorrhage, the histopathology of the marrow as seen in section, presented a characteristic pattern. The densely cellular marrow had clumps of stem cells and basophilic erythroblasts, little or no iron, and a normal cell distribution, except for slight erythroblastic hyperplasia. In 4 cases marrow sections were suggestive but not diagnostic; and in 1 case the marrow was completely dissimilar. Marrow sections of 25 cases of secondary polycythemia, some of which had hemoglobins as high as 20-24 grams, differed from those seen in the primary cases. However, our experience with secondary polycythemia with extremely high hemoglobin is limited.

Myeloid metaplasia developed in 6 patients; 2 had been treated with $\mathrm{P}^{\mathrm{s}}$. Four had extremely cellular marrows and 2 still had active, uncontrolled polycythemia vera. Myelosclerosis was found in 1 terminal patient.

Clinical control (normal peripheral blood, including normal platelet count, and disappearance of abnormal signs and symptoms) following $P^{\text {so }}$ in 8 cases, was correlated with a return of the marrow to normal except for a slight residual erythroblastic hyperplasia. Some of these were followed by serial biopsy as long as four years during 3 courses of treatment. Three other cases, which did not respond to the usual dose of $\mathrm{P}^{22}$, had marrows that were unchanged; during this time their hemoglobin, but not platelet count, was controlled by phlebotomies and spontaneous hemorrhages. Two of these three later were well controlled by larger doses of $\mathrm{P}^{\mathrm{n}}$; after which their platelet counts returned to normal and their marrows responded similarly to the other 8 patients.

The Oxygen Pressure Gradient from Alveolar Air to Arterial Blood in Patients with Mitral Stenosis. S. Gillbert Blount, JR, Malcolm C. McCord and Leighton L. Anderson, Denver, Colorado. (Introduced by Harry Gordon).

Pathological alterations in the alveolar-capillary membrane have been described in patients with mitral stenosis. These findings suggested the possibility of an impediment to oxygen transfer at the alveolar-capillary membrane level in patients with mitral stenosis.

The peripheral arterial oxygen tension was determined by the technique of Riley et al., and the alveolar oxygen 
tension was calculated by the method of Lilienthal et al. This permitted a calculation of the alveolar-capillary oxygen pressure gradient. Twenty young healthy persons were studied by these methods to establish the normal values for this altitude. Fifteen patients with mitral stenosis of varying severity were studied in a like manner. All the patients with mitral stenosis were studied by means of cardiac catheterization.

The mean resting alveolar and arterial oxygen tensions in the normal subjects were $80.06 \mathrm{~mm}$. $\mathbf{H g}$ and 74.60 $\mathrm{mm}$. $\mathrm{Hg}$ respectively, thus resulting in a mean alveolarcapillary oxygen pressure gradient of $5.46 \mathrm{~mm}$. $\mathrm{Hg}$. Following exercise, the mean values were $86.05 \mathrm{~mm}$. $\mathrm{Hg}$ for alveolar oxygen tension and $75.00 \mathrm{~mm} . \mathrm{Hg}$ for arterial oxygen tension. The alveolar-capillary gradient was thus $11.05 \mathrm{~mm}$. Hg. In contrast, the mean resting alveolar and arterial oxygen tensions in patients with mitral stenosis were $78.33 \mathrm{~mm} . \mathrm{Hg}$ and $66.04 \mathrm{~mm}$. $\mathrm{Hg}$. The mean alveolar-capillary gradient was $12.29 \mathrm{~mm}$. $\mathrm{Hg}$. After exercise the mean alveolar oxygen tension was $85.02 \mathrm{~mm}$. $\mathrm{Hg}$, and the mean arterial oxygen tension was $68.38 \mathrm{~mm}$. $\mathrm{Hg}$, giving an alveolar-capillary oxygen pressure gradient of $16.64 \mathrm{~mm}$. $\mathrm{Hg}$.

The differences in the alveolar-capillary oxygen pressure gradient in these two groups indicate a physiologic counterpart to the known pathological alterations in the alveolar-capillary membrane in patients with mitral stenosis. The differentiation of the components of the total alveolar-capillary oxygen pressure gradient is considered. The severity of the mitral stenosis as determined clinically and by cardiac catheterization is correlated with the alveolar capillary oxygen pressure gradient.

\section{The Pressor Action of Atropine in Veratrum Alkaloid}

(Veriloid ${ }^{(R)}$ ) Induced Hypertension. Alfred A. BoloMey and Rosemarie Lenel, Oakland, California. (Introduced by Eaton M. MacKay).

The recent literature would indicate that while atropine effectively abolishes the bradycardia induced by veratrum alkaloids, it fails to influence significantly their hypotensive action. Our recent studies, however, indicate that a remarkable pressor response to atropine occurs frequently in hypertensive patients made acutely hypotensive with Veriloid $^{(R)}$.

Forty-five experiments were carried out on 13 hypertensive patients. Veriloid was administered orally or intravenously in doses sufficient to produce marked hypotension and bradycardia. At the height of the response atropine ( 0.17 to $0.90 \mathrm{mg}$.) was administered subcutaneously or intravenously. A sustained and significant rise in systolic and diastolic blood pressure was observed in 32 instances with control blood pressures being approached or exceeded in 19 . In 30 studies the pulse rate rose simultaneously while in 4 blood pressure rose before pulse rate. In general, blood pressure and pulse rate changes were parallel in magnitude and duration.

Our studies do not lend themselves to an explanation of this atropine effect. A review of the literature indicates that the doses of atropine used were probably not of sufficient magnitude to increase peripheral resistance. The bradycardia may have been profound enough to depress cardiac output, yet, a reversal of such an effect would not seem sufficient to account for the marked pressor phenomenon observed. The doses of Veriloid ${ }^{(8)}$ used in these studies were usually in excess of the therapeutically recommended dose; yet the same effect was observed in ambulatory patients taking constant and previously well-tolerated amounts.

Since profound hypotension and bradycardia are known to occur suddenly in patients taking constant and usually well-tolerated doses of veratrum alkaloids, it would appear important that hemodynamic studies aimed at elucidr.tion of this mechanism be initiated.

Steroid Diabetes Occurring During Cortisone and Adrenocorticotropin ( $A C T H$ ) Therapy. JOHN J. Bookman; Stanley R. Drachman, Louis I. Schaefer, and David Adlersberg, New York, N. Y. (Introduced by Benjamin Kramer).

Among hundreds of patients treated with Cortisone and ACTH for their basic disease five developed the clinical features of steroid diabetes. None presented glycosuria or hypoglycemia prior to the institution of this form of hormonal therapy; in all the signs of diabetes disappeared with the discontinuation of treatment or with reduction of dosage to minimal maintenance levels, and remained so during a follow up period up to one and a half years.

The group as a whole presented all the known features of steroid diabetes although each patient did not exhibit all the features. Doses of insulin up to 70 to 110 units respectively failed to control the glycosuria in two patients. Ketonuria was observed only in one instance. There was a negative nitrogen balance in the one patient so studied, despite a high level of protein intake. The 17 ketosteroids were low prior to and during ACTH therapy in another individual. All patients were predisposed to diabetes; four patients had a familial background of diabetes, the fifth had a transient glycosuria ten years prior to cortisone treatment and a mild but persistent impairment of glucose tolerance one year after cessation of therapy. This indicates that a primary deficiency of insular function was essential for the precipitation of steroid diabetes. Only one patient developed manifest features of diabetes after cortisone therapy; the four others failed to develop diabetes during cortisone but did so during ACTH administration.

It is suggested that impairment of carbohydrate tolerance developing in well established diabetics during infections, burns and other stress situations may represent instances of steroid diabetes superimposed on pancreatic insular insufficiency. The negative nitrogen balance and the resistance to insulin so characteristic of these "episodes" could be easily explained on this basis.

Excretion of Water and Electrolytes in Patients with Essential Hypertension. WILLIAM A. BrodsKy and Harry N. Graubarth, Louisville, Kentucky. (Introduced by J. Murray Kinsman). 
Osmotic diuresis with mannitol loading was produced in six patients with essential hypertension and without previous evidence of cardiac or renal disease. After abstaining from food and drink for 18 hours, the subjects were excreting urine at rates of flow of $0.98-1.68 \mathrm{cc} /$ $\mathrm{min} / 1.73 \mathrm{M}^{2}$, with a sub-maximal osmolarities of $607-700$ mosm/L. During loading, urine flow increased to as high as $14.5 \mathrm{cc} / \mathrm{min}$, with osmolarity, as low as 375 . Minute losses of $\mathrm{Na}$ and $\mathrm{Cl}$ were $2-2 \frac{1}{2}$ times greater than normal, reaching values of 1.8 and $1.4 \mathrm{mEq}$ per minute, respectively. Calculated values of ideal osmotic work amounted to less than one-half that of normal subjects. Clearance of mannitol, at $63.5 \mathrm{cc}$ per minute, was 25 per cent below normal, while that of pAH, $312 \mathrm{cc}$, was 50 per cent of normal.

The findings of high rates of $\mathrm{Na}$ and $\mathrm{Cl}$ excretion with low rates of filtration are compatible with deranged activity of the renal tubules. Although the exact site of tubular damage is unknown, the defective concentrating ability implies distal, and defective composition of urinary solutes, proximal tubular dysfunction. The effects of primary reduction of the arterial supply to the kidney are those of polyuria and chloruria (Verney). It is possible that similar renal mechanisms are operative in uncomplicated hypertensive disease.

Radioactive Potassium Distribution in Metabolic Disorders. Belton A. Burrows, Milton M. Ashley and JонN H. Sisson, Boston, Massachusetts. (Introduced by Francis C. Lowell).

We have found the same rate of distribution of radioactive potassium into body potassium in normal subject, before and during cortisone administration, and in patients with a variety of metabolic disorders: Cushing's Syndrome before and after subtotal adrenalectomy, Addison's Disease before and after treatment, uremic acidosis before and atter extracorporeal dialysis, hepatic cirrhosis before and after recovery from decompensation, cardiac failure or respiratory acidosis before and after treatment, and severe potassium deficiency hefore and after replacement therapy. In all instances equilibration of the radioactive potassium with body potassium was virtually complete at the end of twenty-four hours. Many of these patients had serum potassium concentrations outside the normal range which were not associated with demonstrable changes in the rate of potassium transport across the cellular membrane.

Metabolic balance studies in depleted patients showed close agreement between the amount of potassium retained during replacement therapy and the increase in the amount of body potassium with which the isotope equilibrated (exchangeable potassium) even when such increments amounted to one-half the pretreatment "exchangeable potassium" value. Thus the measured "exchangeable potassium" closely corresponds with the metabolically active body potassium irrespective of profound metabolic alterations or the size of the potassium pool.

The "exchangeable potassium," related to body weight, was often decreased in chronic illness by up to one-half the normal values (40-50 $\mathrm{mEq} / \mathrm{kilo}$ ), although many of the patients had persistent hyperpotassemia. These measurements indicate either a reduction of intracellular potassium concentration or total cell mass, or a proportionate increase in body fat and water, or combinations of these factors. Therefore, these observations offer an explanation for the debilitating effects of chronic illness in terms of reduced organ and muscle function or mass per unit of body weight.

Pulmonary Vascular Function in Mitral Stenosis. Douglas Carrol and Richard Riley,* Baltimore, Maryland.

The diffusing capacity of the lungs has been used to evaluate pulmonary capillary function in 26 patients with mitral stenosis. The findings have been correlated with hemodynamic measurements obtained by pulmonary artery catheterization. In addition, the distribution of ventilation and circulation throughout the lungs has been studied. The patients have been classified on the basis of normal or low diffusing capacity and normal or abnormal distribution.

A low diffusing capacity was found in all cases studied during exercise and indicates that capillary function was impaired. The reduction in capillary function may be due to the following mechanisms: loss of capillaries, thickening of capillary of alveolar walls, obstruction or vasoconstriction of small pulmonary arteries reducing the number of capillaries perfused with blood. Correlation with the hemodynamic studies shows that the mean pulmonary artery pressure and the pulmonary resistance are inversely related to the diffusing capacity. These correlations point toward changes in the small pulmonary arteries, since these vessels are of prime importance in determining pulmonary vascular resistance and in controlling blood flow through the capillaries.

In general, the degree of physiological abnormality was best correlated with the severity of exertional dyspnea and was poorly correlated with duration of disease, hemoptysis, pulmonary edema, paroxysmal nocturnal dyspnea or cardiac enlargement. Abnormalities of distribution, frequently found at rest, improved during exercise.

Post-operative studies suggest that abnormalities of capillary function are in part reversible.

On the Relation of Copper to the Metabolism of Iron. G. E. Cartwright,* C. J. Gubler, M. E. Lahey and M. S. Chase, Salt Lake City, Utah.

Studies in copper-deficient and in iron-deficient swine indicate that copper is intimately concerned in the metabolism of iron.

Swine deficient in copper, like iron-deficient swine, develop a microcytic hypochromic anemia, normoblastic hyperplasia of the bone marrow, hypoferremia and an increase in the iron-binding capacity of the plasma. The hypoferremia occurs early in the course of the deficiency in spite of the administration of $30 \mathrm{mg}$./kilogram of body weight of iron per day. Within 24 hours after the 
administration of copper there is a marked increase in plasma iron.

By the use of radioactive iron it has been possible to demonstrate that in copper-deficient swine, the absorption of iron from the gastrointestinal tract is decreased. Corroborating this finding is the observation that the iron content of the tissues of the copper-deficient swine is decreased. Impairment of iron absorption does not, however, adequately explain the development of anemia in copper-deficient animals since the anemia does not respond to the parenteral administration of large doses of iron. Furthermore, only about 25 per cent of radioiron administered intravenously is utilized for hemoglobin formation.

Swine deficient in iron accumulate copper in the liver in abnormal amounts. Animals deficient in both iron and copper develop anemia much more rapidly than those deficient in either mineral alone.

These results suggest that in copper-deficient swine the ability to metabolize iron properly is impaired and as a consequence of this an anemia, which is morphologically and chemically indistinguishable from iron deficiency anemia, results.

Physiological Effects of Mechanical Exsuffation in Experimental Obstructive Breathing in Human Subjects. Reuben M. Cherniack and Charles A. Gordon, New York, N. Y. (Introduced by Alvan L. Barach).

Obstructed breathing in dogs has been shown to produce pulmonary congestion and edema. These changes have been attributed to the development of an increased negative inspiratory intra-pleural pressure. The present study is concerned with the physiological effects of mechanical exsufflation on experimental obstructive breathing in human subjects. Mechanical exsufflation, developed as a means of eliminating bronchial secretions, depends on a gradual inflation of the lungs by a pressure of 40 $\mathrm{mm}$. $\mathrm{Hg}$, terminated abruptly by a swift opening valve, allowing the pressure to rise to the atmosphere in 0.04 seconds. The expiratory flow rates in normal individuals are thereby increased to about 65 per cent of those found in a maximally vigorous cough. When dyspnea is produced in human subjects by breathing through a $4.0 \mathrm{~mm}$. orifice the peripheral venous pressure (a useful indication of intra-pleural pressure) is lowered in inspiration, and the volume flow rates in inspiration and expiration are prolonged. With exsufflation, dyspnea was relieved and the following physiologic effects were noted: (1) inspiratory venous pressure was elevated to the normal level, (2) inspiratory volume flow rates were markedly increased and (3) expiratory volume flow rates were increased at the onset of expiration. The significance of these findings is discussed.

Antirheumatic and Metabolic Effects of Hydrocortisone in Rheumatoid Arthritis. WILLIAM S. Clark, Joseph

A. Blais and Henrick O. Tonning, Boston, Mass. (Introduced by Charles L. Short).

Hydrocortisone was administered to six patients with active rheumatoid arthritis, intramuscularly and orally, for four- to twelve-day periods in doses of 150 to $300 \mathrm{mg}$. per day. Cortisone acetate was also administered to four of the patients under identical conditions for purposes of comparison. Observations were made on the subjective and objective manifestations of the disease, emotional phenomenon, eosinophils, steroid excretion, glucose tolerance, sedimentation rates and synovial fluids. Three of the patients were studied on the metabolic ward with mineral and nitrogen balance determinations. The steroid was administered both in the form of the acetate and free alcohol. Hydrocortisone acetate was found to be a potent antirheumatic steroid in doses that produced no appreciable metabolic affect as measured by balance studies and 17 ketosteroid excretion. Hydrocortisone acetate and hydrocortisone, mgm. for mgm., had less effect on the subjective manifestations than cortisone acetate, inducing less euphoria, although their effects on the subjective manifestations were similar. Hydrocortisone acetate was found to be metabolically less active than cortisone acetate when administered intramuscularly in duplicate experiments. These studies suggest a greater dissociation between antirheumatic and metabolic effects of hydrocortisone acetate than of cortisone acetate, and a qualitative difference in the effects of these two steroids on the psyche.

\section{A Hemorrhagic Disorder Caused by Circulating Anti- coagulant in Patients with Disseminated Lupus Erythe- matosus. C. Lockard Conley * and Robrrt C. Hart- mann, Baltimore, Maryland.}

Two patients with disseminated lupus erythematosus had a peculiar hemorrhagic disorder attributable to a pronounced coagulation defect. Whole blood clotting times were prolonged, in one case exceeding 24 hours, and there was prolongation of the prothrombin times. Plasma concentrations of fibrinogen, prothrombin and accelerator globulin were normal. Impaired coagulation was caused by a circulating anticoagulant which retarded conversion of prothrombin to thrombin even in the presence of tissue extract thromboplastin. As little as one part of the patient's plasma added to 200 parts of normal blood significantly prolonged the clotting time of the latter. Unlike heparin, the anticoagulant did not retard the clotting of plasma by thrombin, and its effect was not overcome by protamine or toluidine blue. The anticoagulant was not dialyzable, was not absorbed on barium sulfate, withstood heating at $65^{\circ} \mathrm{C}$ for 30 minutes but was inactivated at $80^{\circ}$ C. Plasma fibrinolytic activity was normal, and the concentration of tocopherol was not increased. Serum globulin concentration as measured by the Howe technique was normal. Attempts to separate the anticoagulant by ammonium sulfate fractionation and by electrophoresis were unsuccessful. The anticoagulant titer decreased in one patient during treatment with ACTH and cortisone. The plasma of both patients contained an auto-hemagglutinin, and the Coombs test was positive in the one case examined. Anemia was present but there was little evidence of increased blood destruction. Both patients had biologic false positive serologic tests for syphilis and positive cephalin flocculation tests. L.E. cells were demon- 
strated in the blood of one patient and the diagnosis was confirmed at autopsy in the other. The condition described is an additional example of a situation in which a prolonged prothrombin time is attributable to a plasma abnormality other than prothrombin deficiency.

Effects of Hexamethonium Bromide upon Hemodynamics of Cerebral and Coronary Circulation in Hypertension. Charles W. Crumpton and Quill R. Murphy, Madison, Wisconsin. (Introduced by William S. Middleton).

Separate studies of the quantitative blood flow through the brain and heart were performed during sustained hypertension and repeated after a significant blood pressure reduction induced by hexamethonium bromide administered parenterally.

Cerebral hemodynamics and oxygen metabolism were studied in six hypertensive subjects in the supine position before and one hour after intramuscular hexamethonium bromide (0.8-1.2 mg./kg.). All subjects exhibited a grade III-IV degree of hypertensive retinopathy (KeithWagener classification). Intramuscular $\mathrm{C}-6$ resulted in a $29 \%$ reduction in mean arterial blood pressure and a $30 \%$ decrease in cerebral vascular resistance without essential change in cerebral blood flow (56 and $57 \mathrm{cc} . / 100$ gm./min.). Cerebral oxygen consumption and cerebral arteriovenous oxygen difference did not change significantly during the period of blood pressure reduction.

Studies of the coronary circulation was performed in anesthetized dogs with renal hypertension (experimental perinephritis) before and thirty minutes after intravenous hexamethonium bromide one mg./ kg. Average mean arterial blood pressure promptly decreased from 178 to $83 \mathrm{~mm}$. $\mathrm{Hg}$. and stabilized at $119 \mathrm{~mm}$. $\mathrm{Hg}$. during the second flow determination. Blood pressure reduction was accompanied by a sinus tachycardia. Cardiac work was decreased due to the reduction in mean arterial blood pressure and cardiac output. Coronary blood flow increased slightly, and coronary vascular resistance was slightly reduced during blood pressure reduction.

Hexamethonium bromide administered parenterally resulted in a significant reduction in mean arterial blood pressure in human subjects with sustained essential hypertension and in renal hypertensive dogs. The cerebral and coronary volume flow was maintained during the period of blood pressure reduction under the conditions described.

Hemodynamic and Renal Changes after Pericardiectomy in a Patient with Calcified Constrictive Pericarditis. James W. Culbertson, William H. Ames, Walter M. Kirkendall, Johann L. Ehrenhaft, Ernest O. Theilen and Steven M. Horvath, Iowa City, Iowa. (Introduced by Horace M. Korns).

An unusual experience with repeated physiological evaluation of dramatic results of pericardiectomy for concretio cordis of unknown etiology in a girl of 16 years is reported. Hospitalization followed progressive disability related to exertional dyspnea and peripheral edema (weight gain from 130 to 156 pounds in 6 months). Vital capacity was $3100 \mathrm{ml}$, venous pressure $230 \mathrm{~mm}$ saline, and Decholin circulation time 28 seconds. Electrocardiogram showed low amplitude QRS complexes. Roentgenkymogram demonstrated practically absent ventricular contraction amplitude. Cardiac catheterization revealed elevated right atrial and pulmonary arterial pressures (32/24 and $55 / 45 \mathrm{~mm} \mathrm{Hg}$, respectively) and low normal resting cardiac output $(5.0 \mathrm{~L} / \mathrm{min}$.). Femoral intra-arterial pressure was $105 / 60$. Glomerular filtration rate $(102 \mathrm{ml} /$ min.) and Tm-PAH (106 mg/min.) were normal, but renal plasma flow was strikingly reduced $(288 \mathrm{ml} / \mathrm{min}$.) and filtration fraction correspondingly elevated (0.35). Adequate therapeutic trial of bed rest, dietary sodium restriction, and Mercuhydrin failed to provoke diuresis.

Spontaneous diuresis occurred promptly after complete pericardiectomy. Within two weeks the patient's weight had fallen sharply to 135 , venous pressure to 140 , circulation time to 13 , and right atrial and pulmonary arterial pressures to $8 / 0$ and $28 / 9$, respectively. Increases had occurred in amplitude of QRS complexes and ventricular border movement, resting cardiac output $(+20 \%)$, and femoral arterial pressure (to 125/75). Renal clearance data had changed strikingly to near normal values: GFR 132, RPF 531, FF 0.25, and Tm-PAH 105.

Subsequently the patient has been asymptomatic and working 10 hours daily as a waitress. Comprehensive studies were repeated 4 and 12 months postoperatively and no evidence of impaired cardiac, respiratory, or renal function found. Cardiac output now increases normally on demand, largely through increase in stroke volume. Review of the hemodynamic changes raises the question whether chronic systemic venous distension may not have been of considerable importance in causing the preoperative renal dysfunction.

Comparison of Some Methods for Studying Fat Absorption Illustrated in a Case of Pancreatic Insufficiency. PERRY J. Culver, W. Warren Point and Milton C. HoffMAN, Boston, Massachusetts. (Introduced by Chester M. Jones).

To date the study of fat absorption in man has lacked adequate methods to show both rate and quantity of fat absorption. Gravimetric balance studies are tedious and yield only quantitative information. Blood fat absorption curves shown by lipid carbon determinations, lipo-krit measurements and chylomicron counts are poor approximations of the rate of fat absorption, since each determination represents the component of absorption, mobilization, utilization and deposition of fat.

In the hope of providing a method to measure both quantity and rate, absorption of olive oil labelled with radioactive iodine was compared with the other methods in a patient with pancreatectomy for pancreatic carcinoma. On a constant daily dietary intake of 180 grams of fat, there was a fecal fat loss of 78 per cent of intake. Remarkably similar was the recovery in the stools of 78 per cent of administered radioactivity following a test meal of $\mathbf{3 0}$ grams of radioactive iodine labelled olive oil. Blood fat absorption curves after this same test meal were flat 
by chylomicron, lipokrit and lipid carbon measurements. A definite rate of fat absorption, however, was shown by the rise in serum radioactivity and recovery of 21 per cent of the administered radioactivity in the urine. By modifying the control conditions, with resultant improvement in fat absorption, the gravimetric method of balance studies correlated well with the radioactive isotope recovery in the stool.

Close correlation between fecal fat losses measured gravimetrically and isotopically plus parallel changes in blood fat absorption curves under the various conditions of this experiment suggest that olive oil labelled with radioactive iodine is a rapid and accurate method for determining both quantity and rate of fat absorption.

Studies of Absorption from the Human Small Intestine by an Intubation Technique: The Effect of Variations in Blood Sugar on the Rate of Absorption of Glucose. Alvin J. Cummins, New York, N. Y. (Introduced by Thomas P. Almy).

The rate of absorption of solutions of glucose $(5 \%$ and $10 \%$ ) and methionine $(0.25 \%$ and $0.50 \%)$ from the upper small intestine was measured in 17 subjects, using a modified Nicholson-Chornock multiple lumen tube. These solutions were delivered into the intestine at constant rates, and the unabsorbed residue was aspirated $45 \mathrm{~cm}$. below the point of inflow.

The absolute rates of absorption for glucose and methionine, as determined by this method, varied widely from person to person. In 7 subjects, the greatest variation in absorption rate in two identical and consecutively performed tests was $25 \%$ for glucose and $50 \%$ for methionine.

Cori found in experiments on rats that the absorption of $25-80 \%$ glucose was independent of the concentration fed. Within the more physiologic range of concentrations studied by us, the rate of absorption of both glucose and methionine was significantly greater from the more concentrated solutions. This indicates that the human small intestine may not conform to "Cori's law" of glucose absorption.

Production of hyperglycemia by intravenous glucose or of hypoglycemia by intravenous insulin failed to alter significantly the rates of absorption for glucose and methionine.

Observations on the Use of Protoveratrine in Hypertensive Vascular Disease. J. H. Currens, G. S. Myers and J. S. McGinty, Boston, Mass. (Introduced by E. F. Bland).

Twenty-four patients have been observed from six to thirty months (average thirteen) while receiving protoveratrine in an effort to control hypertension. Ten patients described hypertension headaches and in nine the relief was classified as good. Five patients had symptomatic hypertensive heart disease all of whom had significant improvement.

Seven patients with toxemia of pregnancy have received protoveratrine for three to eight weeks with apparent beneficial effect on blood pressure and course of pregnancy.
Protoveratrine was administered orally usually after meals and bedtime, in dosage ranging from $0.4 \mathrm{mg}$. to $1.5 \mathrm{mg}$. Enteric coated protoveratrine at bedtime was useful to control early morning hypertension. Adverse symptoms such as nausea, vomiting, salivation, giddiness, and weakness were noted by all patients at some time during treatment one half to two hours after medication. Delicate regulation of individual dosage by $0.05 \mathrm{mg}$. usually adjusted by patient, reduced these adverse reactions to a minimum and infrequently precluded continuous administration. The patients noticed transient hemiparesis following emesis at time of maximal sedative effect. No significant sequelae have been observed after toxic reactions. One patient received 50 mgs. without disaster.

Renal clearance of para-amino hippuric acid after intravenous protoveratrine was observed to decrease in the first thirty minutes associated with abrupt fall of blood pressure. Clearance values usually returned to control levels during two subsequent thirty minute periods with blood pressure still reduced.

Cardiac catheterization has revealed significant fall in cardiac output in most patients after intravenous protoveratrine. Decrease in mean blood pressure and stroke output after protoveratrine results in appreciable fall in left ventricular work index. Elevated pulmonary arterial pressure decreased to normal after protoveratrine. Atropinization in two patients failed to prevent significant slowing of heart rate after protoveratrine.

Protoveratrine administration is feasible as long term treatment of hypertensive vascular disease in some patients.

The Influence of the Tonicity of the Body Fluids on the Rate of Excretion of Electrolytes. J. H. DARRAGH, L. G. Welt, A. V. N. Goodyer and W. A. Abele, New Haven, Conn. (Introduced by David M. Kydd).

The influence of the tonicity of the body fluids on the rates of excretion of sodium, chloride, potassium, and ammonia was examined in four healthy young adult male subjects.

An infusion of an isotonic or hypertonic solution of sodium chloride was administered after a control period during which the tonicity of the body fluids was either: 1) not altered, or 2) reduced by achieving and maintaining a positive balance of water of about two liters. This latter was accomplished with infusion of a solution of five per cent glucose in water. Pitressin was included in the glucose infusion to reduce urine volume to a minimum and thus to facilitate the maintenance of the positive balance of water. Each of the four subjects served as his own control and was studied with and without dilution of the body fluids. Similar amounts of pitressin were given in the control studies.

The rates of excretion of sodium and potassium were lower in the dilution studies than in the control experiments where the tonicity of the body fluids had not been decreased. In contrast, the rate of excretion of ammonia was higher in the dilution studies.

The slower rate of excretion of sodium in the dilution studies was influenced, presumably, by the decrease in the 
effective osmotic pressure of the extracellular fluid or its necessary consequence-an increase in cellular hydration. Since cellular dehydration is alleged to augment the excretion of potassium, it may be that the diminished rate of excretion of this ion in the dilution studies is due to cellular overhydration.

The increase in the rate of excretion of ammonia may have been the consequence of the demand for the continued excretion of cation at a time when there was a diminished rate of excretion of sodium and potassium.

A Disturbance in Inositol Metabolism in Diabetes Mellitus. W. H. Daughaday,* J. Larner and Ellabeth Houghton, St. Louis, Missouri.

Although increased excretion of inositol in diabetes mellitus has been reported several times since 1864, this observation has been generally overlooked or attributed to polyuria. Now that inositol has been found to be a structural component of cephalin and probably is concerned with hepatic lipid metabolism, its increased excretion in diabetes mellitus merits reinvestigation.

The daily inositol excretion of five normal subjects on an unrestricted diet has been measured microbiologically using S. Carlsbergensis. The average inositol content of 15 urine specimens examined was $56 \mathrm{mg}$ per day (range 26 to $144 \mathrm{mg}$ per day). Two normal subjects excreted an average of $30 \mathrm{mg}$ per day of inositol with an average urine volume of $907 \mathrm{ml}$. When the urine volume was increased to $2782 \mathrm{ml}$, the inositol excretion rose to only $46 \mathrm{mg}$ per day.

Repeated observations were made of the excretion of inositol by six hospitalized patients with uncontrolled diabetes mellitus. In every case the inositol excretion was high. The maximum excretion by each patient was 280 , $341,350,518,581$ and $851 \mathrm{mg}$ per day. The amount of inositol found in the urine correlated well with the degree of glycosuria. When glycosuria was adequately controlled by insulin treatment the excretion of inositol fell toward normal. In both the normal and diabetic subjects the urinary inositol appeared to be in the free form.

Similar changes in inositol excretion have been observed in partially depancreatized rats. Inositol excretion in these animals is largely dependent on dietary inositol. At any given oral intake the diabetic rats excreted much more inositol than did the normal animals.

It is concluded that there is a defect in inositol metabolism in diabetes mellitus which cannot be explained by differences in inositol intake or urine volume.

Separation of Primary Amines from Urine and Blood. Dean F. Davies and Kathryn M. Wolf, St. Louis, Missouri. (Introduced by John R. Smith).

Methods developed in this laboratory for the demonstration and separation of primary amines have been used in the study of their presence in normal, pooled urine, and in 24 hour collections of normal and hypertensive urine. Samples of pooled male urine collected under toluene were brought to $\mathrm{pH} 10$ and centrifuged. Continuous extraction of the supernatant was carried on for two four-hour periods, the urine hydrolyzed in acid, made alkaline again, and re-extracted four hours. Concentrates of the extracts were chromatographed by the ascending technique and sprayed with specially prepared ninhydrin solution. Six hitherto unrecognized amine substances were identified and, by previous experience with known amines, their nature could be inferred. The heaviest and most consistent appears to be a 4-6 carbon aliphatic primary amine having a prolonged pressor action. Its $\mathrm{Rf}$ is close to that of isoamylamine $(0.5)$, but evidence against their identity include electron diffraction patterns and characteristics of color development. Other urinary amines appear either to be of shorter chain length or to contain hydrophilic groups.

Attempts to correlate the appearance of these compounds with the hypertensive state in twenty-two 24-hour samples from six subjects indicate no distinct difference between normotensives and individuals with benign hypertension. However, malignant hypertensives have altered patterns. One patient with the endocrine hypertensive syndrome had large quantities of a histamine-like amine. Administration of organic nitrates when compared with control periods on placebo appears to decrease the output of amines in non-endocrine individuals.

In blood, several amines appear and two are interpreted to have longer aliphatic moieties than isoamylamine. One may be a small volatile pressor substance previously found.

\section{Evidence for an Altered Enzyme Resulting from Mutations} to Drug Resistance or Nutritional Requirement. Bernard D. Davis * and Werner K. MaAs, New York, N. $Y$.

Conceivable biochemical mechanisms of drug resistance include: $(1,2)$ decreased penetration or increased destruction of the drug; (3) increased synthesis of an antagonistic metabolite; (4) increased concentration of the enzyme utilizing this metabolite; (5) more efficient utilization of the product of this enzyme; (6) an alternative metabolic pathway; and (7) formation of an enzyme with decreased affinity for the drug or increased affinity for the metabolite.

Discrimination among these mechanisms became possible with the discovery that $p$-nitrobenzoic acid (PNB) inhibits Escherichia coli by two independent mechanisms, competing simultaneously with $p$-aminobenzoic acid (PAB) and $p$-hydroxybenzoic acid. In the presence of PNB plus either metabolite, mutants resistant to PNB competition with the other metabolite could be selected. Since these two types of mutants were found not to be cross-resistant, mechanism 1 and 2 are excluded for these strains. Furthermore, resistance to $\mathrm{PNB}$ competition with $\mathrm{PAB}$ was found to differ from resistance to sulfonamide competition with PAB. This finding excludes mechanisms 3 to 6 , which could not discriminate between different analogues. These results therefore point to either mechanism 7 or some unknown mechanism.

Further, more direct support for an altered enzyme was next provided by the observation that increased resistance to a sulfonamide is associated with a proportional increase 
in the sulfonamide/PAB competitive ratio. Finally, while a rigorous proof of enzyme alteration was not possible for drug resistance, since the PAB-utilizing enzyme could not be studied in extracts, conclusive proof for another kind of qualitative alteration has been achieved with another enzyme: the pantothenate-synthesizing enzyme extracted from a mutant which requires pantothenate only at temperatures above $30^{\circ}$ differs from the corresponding wild-type enzyme in being extraordinarily sensitive to heat denaturation.

This ability of mutations to lead to qualitative as well as apparently quantitative changes in enzyme production is significant for other medical problems besides drug resistance, such as hereditary diseases, neoplastic growth, and variations in microbial virulence.

The Acceleration of Clotting by Platelets Treated with

Thrombin Preparations. JANE F. Desforges and Frederick S. Bigelow, Boston, Mass. (Introduced by Clark W. Heath).

Platelets plus a plasma factor are known to produce a thromboplastic effect in blood coagulation. To determine whether there is enhanced platelet activity once thrombin is formed, the effect of platelets treated with various thrombin preparations was observed in several phases of clotting.

Platelets washed in saline were incubated at room temperature with thrombin preparations and then washed to remove free thrombin. These platelets were found to shorten whole blood clotting and recalcification times to a degree not observed with washed untreated platelets.

The effect of treated platelets on prothrombin consumption and on two-stage prothrombin tests demonstrated increased prothrombin consumption and acceleration of thrombin formation in normal and in hemophiliac plasma. This action was evident in the absence of added thromboplastin but was not demonstrable either with thrombin preparations or with untreated platelets alone.

Thus, washed platelets exposed to thrombin possess thromboplastic activity not noted with untreated platelets. This action of treated platelets suggests a further means by which coagulation proceeds at an increasing rate.

The treated platelets showed no consistent change in stickiness, agglutination, electrophoretic mobility or microscopic appearance.

Urinary Alpha-Ketolic Corticoids Determined by Paper Chromatography in Patients with Hypo- and Hyperfunction of the Adrenal Cortex. F. C. DoHAN * and E. M. Richardson, Philadelphia, Pennsylvania.

The alpha-ketol group is characteristic of metabolically active corticoids and some of their metabolites. This study has demonstrated the expected marked decrease in urinary excretion of the alpha-ketolic steroids in patients with Addison's or Simmond's Disease and the marked increase in Cushing's Syndrome. The pattern of excretion in Cushing's Syndrome is similar to that produced by intravenous injection of ACTH. The urinary steroids were hydrolyzed by glucuronidase followed by acid hydrolysis during chloroform extraction. Paper chromatography was performed by a modification of the methods of Zaffaroni, Burton, and Keutmann and of Tennent, Whitla, and Florey. This permits the use of a small aliquot of the 24 hour urine collection for the detection (with blue tetrazolium) and semi-quantitative measurement of steroids containing the alpha-ketol group. Identification has, in general, followed the technics of Zaffaroni et al.

Following the injection of 10-20 mg. of ACTH intravenously in two hypertensive men, at least 9 alpha-ketols have been detected in the 3 chromatographic fractions. In the most polar fraction, tetrahydrocortisone and a closely associated, highly polar compound (probably tetrahydro-hydrocortisone), reduction products of compounds " $E$ " and " $F$ ", are excreted in large amounts in normal individuals, and found increased in Cushing's Syndrome, and greatly increased after ACTH. Hydrocortisone (" $F$ ") was found in markedly increased amounts in Cushing's Syndrome and after ACTH injection. At least two alpha-ketols are found in the cortison ("E") region after ACTH and possibly in Cushing's Syndrome. Further identification studies are in progress.

After ACTH, and in Cushing's Syndrome, 3 alphaketols have been detected in the second fraction, and one in the third, least polar fraction. Those in Cushing's Syndrome appear to be similar to those found after ACTH administration. Little or none of these substances was found in the urine of untreated patients with Addison's or Simmond's Disease.

\section{Microscopic Correlation of Vascularity with Glandular Secretion in the Stomach of Man in Vivo. RoNALd K. Doig and Stewart Wolf,* New York, New York.}

In the fistulous subject, Tom, it has been possible with a dissecting microscope to examine and photograph the venous channels which surround the necks of the simple branched tubular glands in the body of the stomach. Simultaneous measurement of the production of acid, pepsin and glandular mucoprotein showed a direct correlation between vascularity and glandular function. The variation in size of the vascular channels between the state of hypersecretion and of glandular hypofunction with anacidity was greater than 100 per cent. It is of incidental interest but of unexplained significance that the membrane fluoresces under filtered ultraviolet light in the presence of active glandular function and that the extent and intensity of the fluorescence is roughly proportional to glandular function. Colored drawings of the fluorescence will be shown as well as photographs in color of the microscopic appearance of the gastric mucosa for the first time in a human being in vivo.

\section{Prevention of Experimental Arthritis by Combined Treat- ment with Phenergan and Irgapyrine. PIERre Ducom- MUM, Montreal, Canada. (Introduced by Hans Selye). \\ On the basis of previous experiments concerned with the anti-inflammatory potency of some anti-pyretics and anti-histaminics, we have studied the anti-phlogistic prop- erties of the association of Phenergan (dimethyl-amino-}


2-methyl-1 ethyl $\mathrm{N}$ di-benzoparathiazine) and Irgapyrine (dipyrine and 3,5,-dioxo-1,2 diphenyl-4-n-butyl-pyrazolidin-Natrium) upon experimental arthritis in intact and adrenalectomized rats.

The development of the tropical arthritis was prevented under the influence of this combined treatment with Phenergan (6 $\mathrm{mg}$ ) and Irgapyrine (30 $\mathrm{mg})$, subcutaneously injected once daily. The prevention observed was almost as complete as that obtained with cortisone treatment (2.5 $\mathrm{mg}$ daily).

Further investigations showed that this anti-arthritic action could be produced in adrenalectomized rats, thus indicating that hypophyseal adrenal stimulation was not responsible for the anti-phlogistic effect.

Other combinations of such related compounds were investigated, but Phenergan-Irgapyrine was found to be the most potent association.

The parallelism between the action of cortisone and Phenergan-Irgapyrine is also found on the connective tissue permeability level: Phenergan-Irgapyrine, like cortisone, decreases the spreading of subcutaneously injected hemoglobin in intact and adrenalectomized rats.

Under the influence of this combined treatment, the hepatic glycogen content in fasting adrenalectomized rats remains unchanged, while the anti-arthritic effect is prominent.

We report these results merely to stimulate interest in the possible clinical applications of such drug combinations.

Endogenous Fat Metabolism Studied by Heavy Water Dilution and Nitrogen Balance. I. S. EDELMAN, L. Brooks, and F. D. Moore,* Boston, Mass.

Previous studies from this laboratory have established the feasibility of measuring total body water in the normal living subject by deuterium oxide dilution. We have now extended our work on body water to patients suffering from a variety of illnesses. We find that this method, when combined with nitrogen balance, yields information on fat metabolism. This forms the subject of the present communication.

The human body is composed of fat, lean tissue solids and water. The mineral fraction of the body is about $7 \%$ by weight, largely situated in the skeleton. Carbohydrate totals less than $1 \%$ of body weight. Estimation of in vivo changes in body fat content is therefore made possible by direct measurement of the nitrogen balance and total body water, and calculation of the fat changes by the following equations.

(1) Body weight $=$ fat + lean tissue solids + water.

(2) $\Delta$ lean tissue solids $=\Delta$ nitrogen balance $\times 8.1$.

(3) $\Delta$ body fat $=\Delta$ body weight $-(\Delta$ water $+\Delta$ lean tissue solids).

Alternative expressions for the interrelationships of fat, water and lean tissue have evolved from the work of Pace and Behnke on body fat content and specific gravity. These expressions are valid in the absence of changes in hydration. The above equations are generally valid, however, even where acute disease is present and are independent of changes in lean tissue hydration.

A series of studies will be reported in which changes in body water, lean tissue solids and body fat were measured by these methods. Utilization of fat at rates up to 170 grams per day (a caloric equivalent of 1530 calories per day) is not uncommon in acutely ill patients. Fat redeposition in convalescence usually occurs at a rate in the vicinity of $\mathbf{5 0}$ to $\mathbf{7 5}$ grams per day.

Composition and Distribution of Plasma Lipoproteins in Normal and Pathological States. How ARD A. EDER and ElLA M. Russ, New York, N. Y. (Introduced by David P. Barr).

Recent studies have shown that essentially all the cholesterol and phospholipid of the plasma are combined with protein either as alpha or beta lipoproteins. When these lipoproteins are separated by Cohn fractionation, their composition can be studied and the distribution of the plasma lipids between the two groups determined. In atherosclerosis as well as in nephrosis and diabetes, the distribution of cholesterol has been found to be abnormal. In the present study the distribution of the other plasma lipids has been determined in order to understand better the role of lipoproteins in the pathogenesis of these diseases.

In normal fasting individuals about one third of the plasma neutral fat was found in alpha lipoprotein, and the remainder was in beta. When plasma concentration of neutral fat was increased either by ingestion of neutral fat or in disease such as nephrosis, the increment was found almost entirely in beta lipoprotein. Following the administration of heparin, there was no change in the total plasma lipids or in the distribution of cholesterol or phospholipid. The effect of heparin on visible lipemia must therefore be considered in terms of its effect on neutral fat distribution.

In patients with biliary cirrhosis and in the early stages of acute hepatitis, the lipoproteins are qualitatively different from those found in normals and in all other diseases thus far studied. In beta lipoprotein the ratio of cholesterol to phospholipid was less than half that usually found and approached the ratio found in alpha lipoprotein. Normally the ratio of non-esterified to total cholesterol in the lipoproteins varies only slightly from that of whole plasma. In these patients the cholesterol of alpha lipoprotein may all be free cholesterol with beta lipoprotein containing all the esterified and some free cholesterol. With recovery from hepatitis the lipoproteins return to the normal pattern.

\section{Hemodynamic Studies in a Case of Tricuspid Stenosis. M. Irene Ferrer,* Rejane M. Harvey and Andre Cournand, New York, N. Y.}

The hemodynamics of the circulation in a patient with sinus rhythm and tricuspid stenosis of rheumatic origin, proven later by necropsy, were studied using the cardiac catheterization technique. The pressure curves in the right auricle and ventricle-the compartments on either 
side of the stenosis-provide the background for a discussion of the detailed performance of these chambers. As might be expected, the pressure in the right auricle remained higher than in the right ventricle throughout diastole. Three other unusual discrepancies were noted: (a) the end diastolic point in the right auricle and ventricle were not identical, as occurs in normal or even dilated hearts, and (b) "isometric" contraction of the right ventricle was in progress for a longer than normal time before the tricuspid valve closed, and finally (c) there was no visible "c" wave in the auricular tracing, eliminating the possibility of significant tricuspid regurgitation and indicating that there was probably downward fixation of the leaflets preventing an upward systolic bulge. Other features of the curves indicated continuous though varying atrioventricular flow throughout diastole and absence of early rapid diastolic ventricular filling. The response to exercise in this patient, who also had mitral stenosis, suggested that the lack of increase in cardiac output during effort may have been due to the tricuspid stenosis itself which impounded the increasing venous return in the auricle and largely prevented this increase from reaching the ventricle or pulmonary bed. Hence the rise in pulmonary artery pressures during exercise in this woman could be ascribed only to the effects of increased heart rate in face of a mitral stenosis.

Since autopsy findings permitted the measurement of the area of the stenosed tricuspid valve, the hydraulic formula for estimation of valve area was applied using the hemodynamic data. The observed and calculated valves were not identical.

\section{The Significance of Plasma Iron Turnover. STUART C.} Finch and JosepH F. Ross,* Boston, Massachusetts.

The plasma iron has physiological significance, since it is iron in transport and is in dynamic equilibrium with other fractions of body iron. Variations in plasma iron concentration reflect physiological and pathological variations in iron metabolism and hematopoiesis. The rate of turnover of plasma iron is of greater potential interest than is the plasma concentration, since turnover rates may be expected to provide fundamental information relative to the kinetics of iron metabolism and hematopoiesis, and may have definite clinical diagnostic value.

Employing radioiron as a biologic tracer, we have investigated the kinetics and clinical significance of plasma iron turnover in human subjects and in dogs. The influence on plasma iron turnover of alterations in rates of erythrocyte production, in plasma iron levels, in size of total body iron stores, and in the functional capacity of the reticuloendothelial system has been evaluated.

Our studies indicate that the amount of plasma iron turnover is primarily a reflection of the amount of red blood cell formation. The rate of plasma iron clearance also is primarily a reflection of the red blood cell formation and is only secondarily affected by reticuloendothelial function and plasma iron level. Other factors exert a definite but small influence on plasma iron turnover. The rate of erythrocyte formation is reliably indicated by this amount of plasma iron turnover and this determination provides a rapid and useful method of assessing erythropoietic rates. Finally, it becomes apparent that no reliance can be ascribed to oral plasma iron tolerance tests as an index of iron absorption, unless the plasma volume and iron clearance rates are known.

Failure of Equilibration of Inulin in Anuric Subjects. John T. Finkenstaedt, Maurice P. O'Meara and JoHn P. MERrIII, Boston, Massachusetts. (Introduced by James P. O'Hare).

Measurement of the volume of distribution of inulin following a single injection was attempted in 14 anuric patients and 4 nephrectomized dogs. Failure to equilibrate was observed since serum inulin levels fell continuously. This could not be explained by urinary or other losses, or by interfering substances in the sera. All subjects had restricted fluid intake and lost weight.

In 12 patients given 4.5 to $13 \mathrm{gm}$. of inulin, the apparent "inulin space" at 8 hours averaged 24\% (20.8-31.0\%) of body weight. The 24 hour inulin space in 10 patients averaged $30.2 \%(23.5-37 \%)$. Spaces of 4 patients followed for 96 hours averaged $45.2 \%(31.7-53.6 \%)$. In 2 of these patients, 8 hour spaces determined at 48 hours, after a second injection of inulin, were similar to those after the initial dose. Preinjection inulin blanks ranged from 2.8-9.5 mgm.\%. Urinary recoveries during diuresis were inaccurate because of high urinary blanks.

In 3 bilaterally nephrectomized dogs given large doses of inulin $(1.0 \mathrm{gm} / \mathrm{Kg}$.) inulin concentrations fell continuously over 5-7 days of survival without exogenous loss. The rate of disappearance of inulin averaged $0.5 \%$ of the administered dose per hour. By 150 hours, the apparent "inulin spaces" were $50 \%$ of body weight. In 1 dog given a dose more comparable to the patient dose $(0.2 \mathrm{gm} / \mathrm{Kg}$. $)$, the serum level approached the blank value in 120 hours.

It is concluded that inulin cannot be used to measure "extracellular space" in anuric subjects because of its failure to equilibrate. Whether this is the result of catabolism, transfer into cells, entry into the reticuloendothelial system or other compartments is unknown.

The Effect of Corticotropin Administration on Blood Bile Acids and Pruritus in Biliary Cirrhosis. EDMUNd B. Flink and Charles F. Williams, Minneapolis, Minnesota. (Introduced by F. W. Hoffbauer).

Corticotropin was administered to four patients with primary biliary or cholangiolitic cirrhosis and one patient with secondary biliary cirrhosis. The most significant alterations were a decrease in blood bile acids and a decrease in pruritus.

Before corticotropin administration the blood bile acid concentrations were elevated to two to three times the upper limit of normal value $(1.0 \mathrm{mg} . / 100 \mathrm{cc}$.). During treatment of the first patient the bile acid level fell in 12 days from $2.0 \mathrm{mg} . / 100 \mathrm{cc}$. to $0.8 \mathrm{mg} . / 100 \mathrm{cc}$. and pruritus disappeared. One month later the pruritus returned to its previous severity and the bile acid concen- 
tration had risen to $2.3 \mathrm{mg} . / 100 \mathrm{cc}$. Therapy for 7 days caused a drop in bile acids to $1.2 \mathrm{mg} . / 100 \mathrm{cc}$. and nearly complete alleviation of the pruritus. The second patient experienced only slight diminution in pruritus, and the bile acid level decreased from $2.7 \mathrm{mg} . / 100 \mathrm{cc}$. to $2.0 \mathrm{mg}$./ $100 \mathrm{cc}$. in 12 days. The third patient had only mild pruritus initially, but it diminished during therapy. The bile acid level decreased from $3.0 \mathrm{mg} . / 100 \mathrm{cc}$. to $2.0 \mathrm{mg}$./ $100 \mathrm{cc}$. in 8 days. The fourth patient noted nearly complete relief from pruritus and the bile acids decreased from 3.6 to $2.1 \mathrm{mg} . / 100 \mathrm{cc}$. The fifth patient (secondary biliary cirrhosis) noted complete relief from pruritus, and the bile acids decreased from $3.2 \mathrm{mg} . / 100 \mathrm{cc}$. to $1.6 \mathrm{mg} . / 100 \mathrm{cc}$.

The decrease or disappearance of pruritus in these patients at a time when bile acid concentrations were decreasing rapidly and a return of pruritus when bile acid levels rose again are in accord with the concept that bile acids have some relationship to pruritus. The decrease in bile acids cannot be accounted for by a decrease in the degree of regurgitation jaundice. It is possible that bile acids were used in the synthesis of adrenal corticoids or that an increased biliary excretion was induced.

The Effect of the Continuous Removal of Measured Amounts of Spinal Fluid on Cerebro-Spinal Fluid Dynamics. F. F. Foldes, E. Keutmann and R. D. Hunt, Pittsburgh, Pennsylvania. (Introduced by $\mathbf{P}$. C. Zamecnik).

0.2 cc., 0.4 cc., 0.6 cc., and 0.8 cc. of spinal fluid per minute were removed from four patients each over a 60 minute period. In another four patients $3 \mathrm{cc}$. per minute of spinal fluid were removed over a 20 minute period. The cerebro-spinal fluid pressure, pulse rate, and blood pressure were observed every 5 minutes. At the end of the experiments the original cerebro-spinal fluid pressure was re-established by the subarachnoidal injection of normal saline. When 0.2 to $0.4 \mathrm{cc}$. of spinal fluid per minute were removed, after an initial decrease, the level of the spinal fluid pressure became stabilized. When larger quantities of spinal fluid were removed, there was a progressive decline in the spinal fluid pressure all through the experiment. When $3 \mathrm{cc}$. of spinal fluid per minute were removed, the spinal fluid pressure dropped from an average of $200 \mathrm{~mm}$. to $10 \mathrm{~mm}$. of water in 20 minutes. It was found that consistently less saline was necessary to re-establish the original spinal fluid level than the amount of spinal fluid removed in the course of the experiment. The relationship between the amount of spinal fluid removed and the amount of saline necessary for the re-establishment of the original spinal fluid level provided interesting data on some aspects of the mechanism of spinal fluid secretion.

Ventilatory Function in Mitral Stenosis. RoBerT N. Frank, David W. Cugell and Edward A. Gaensler, Boston, Mass. (Introduced by Laurence B. Ellis).

The ventilatory effects of pulmonary vascular changes are well demonstrated in mitral stenosis. This disease usually evolves in two distinct phases: Initial pulmonary hypertension without increment in blood volume, and terminal congestive circulatory failure with increase in blood volume.

Sixty-two patients were studied in whom mitral stenosis was the predominant valvular lesion. They were classified according to the New York Heart Association criteria of functional limitation. Patients with complicating pulmonary disease were excluded.

In patients with asymptomatic mitral stenosis, all measurements of pulmonary function during rest and exercise were normal. With increasing disability there was progressive reduction of maximum breathing capacity, total and timed vital capacity. In severe disability, the total lung capacity was decreased chiefly at the expense of the vital capacity. The associated relative increase in residual volume was not attended by a defect in intrapulmonary mixing. Patients with slight limitation of activity experienced discomfort during walking exercise. However, they did not hyperventilate. The breathing reserve was not significantly depleted until disability was marked.

By comparison, patients with pulmonary disease with equivalent degrees of incapacity showed a greater ventilatory defect. In restrictive insufficiency the maximal breathing capacity, vital capacity, and total lung capacity showed greater reduction than in mitral stenosis. In obstructive insufficiency, the maximal breathing capacity was less, the total lung capacity and residual volume greater, and the intrapulmonary mixing index abnormally elevated. The breathing reserve in pulmonary disease was characteristically smaller. The patients with mitral stenosis, unlike those with pulmonary disease, were disabled disproportionately to the measured defects in pulmonary function.

The results were ascribed to two separate mechanisms. Initially, pulmonary hypertension reduced lung compliance and interfered with maximal flow velocity. With the advent of circulatory failure and blood volume increase, the total lung capacity was reduced.

The Thyroid Hormonal Pool in Man and Dog. A. Stone Freedrerg,* Leonard Wolsky, George S. Kurland and Mirton W. Hamolsky, Boston, Massachusetts.

Radioactive iodine studies have made significant contributions to our understanding of many aspects of thyroid function. Available methods, however, have not yielded conclusive information concerning certain basic problems. The infusion of labelled thyroid hormone was utilized to study two such problems: (1) The size and turnover of the hormonal pool in normal and abnormal states of thyroid function and (2) the nature of the hormone elaborated by the thyroid gland in various metabolic states. The thyroid hormonal pool in man and dog was studied by determining the rate of disappearance of protein-bound In ("radioiodothyroglobulin") obtained from humans treated by $\mathrm{I}^{12}$. Following intravenous infusion of such plasma, recipient's blood was analyzed frequently over 23-70 hours for remaining total and protein-bound $I^{12 x}$. In each experiment there was a rapid 
initial exponential decrease and a slower terminal exponential decrease. Standard Graphic analysis revealed: I: In human recipients, the average value for the total exchangeable "thyroglobulin" ( $\mu$ gms) in four euthyroid subjects was $399 \pm 44$ vs. 1142 in a thyrotoxic and 65.0 in a patient with myxedema. Circulating "thyroglobulin" ( $\mu \mathrm{gms}$ ) averaged $173.5 \pm 23.8$ in 4 euthyroids vs. 557 in the thyrotoxic and 23.0 in the myxedemic patient. The turnover of total "thyroglobulin" ( $\mu \mathrm{gms} /$ day) averaged $119.4 \pm 11.4$ in the euthyroids vs. 480 for the thyrotoxic and 26.2 for the myxedemic patient. II. In dog recipients, following infusion of such tagged plasma from 2 euthyroids the turnover time was 37.9 and 30.2 hours, the half-time was 26.3 and 21 hours and the turnover rate, 63.2 and $79.2 \% /$ day. Infusion of plasma from 2 thyrotoxics yielded turnover times of 18.1 and 17.8 hours, half-times of 12.6 and 12.4 hours and turnover rates of 132.0 and $134.0 \% /$ day. These observations demonstrate a clear difference in the size of the exchangeable thyroid hormonal pool, as well as in the daily turnover, in various states of thyroid function. They also denote a basic difference in the nature of the thyroid hormone elaborated by patients with thyrotoxicosis as contrasted with euthyroid individuals.

A New Method for Measuring Transcapillary Exchanges: The Transfer of Salt and Water in the Lesser Circulation of Man. NORBERT FreINKeL, George E. Schreiner and John W. Athens, Washington, D. C. (Introduced by Robert W. Berliner).

The Stewart Principle has been modified for the evaluation of net exchange of micromolecular solutes across capillary beds. Flow measurements based upon the mean dilution of an indicator during its first circulation will be artifactually elevated if a portion of the indicator is diverted from the stream during that circulation. Hence, if a crystalloid is administered simultaneously with a colloid, differences in blood flow determined separately from their concurrent dilutions must reflect the net extravascular (i.e. transcapillary) loss of the more diffusible agent.

This technique of simultaneous indicators was applied to a study of net salt and water transfers in the lesser circulation of man. T1824 was employed as the intravascular standard. Such use of T1824 was validated by the demonstration of instantaneous and complete dyeprotein union during the first circulation.

$\mathrm{T} 1824$ in various combinations with $\mathrm{I}^{1 \mathrm{x}}$-labelled albumin, inorganic $\mathrm{I}^{2 \mathrm{x}}, \mathrm{Na}^{24}$ and $\mathrm{D}_{2} \mathrm{O}$ was administered to 24 subjects. Delivery was made through a catheter within the subclavian vein. Flow values were calculated from the extrapolated semilogarithmic plot of arterial concentrations during the first circulation. The blood flow ratio T1824 Indicator/Crystalloid Indicator was used as an index of relative "virtual" distribution compartments. A ratio of unity denotes no net transfer. After correction for red cell penetration, T1824/Inorganic $I^{13}$ flow ratios averaged $1.015( \pm 0.03)$ and $T 1824 / \mathrm{Na}^{24}$ ratios averaged $0.997( \pm 0.04)$. This method did not disclose any significant transcapillary exchange or extravascular dilution of electrolytes within the lesser circulation of normal man.

The $\mathrm{T} 1824 / \mathrm{Na}^{24}$ flow ratio in a patient with mitral stenosis and proven pulmonary hypertension was 0.902 . In another subject with a large carotid arteriovenous fistula, the T1824/Inorganic ratio was 0.881 . Both values are suggestive of transcapillary electrolyte exchange. Potentialities of the simultaneous indicator technique in abnormal states will be discussed.

The Hemodynamic Effects of Hexamethonium in Man. Edward D. Freis, John C. Rose, Thomas F. Higgins, Roberts T. Kelley, Harold W. Schnaper and Robert L. Johnson, Washington, D. C. (Introduced by Harold Jeghers).

The reduction of arterial pressure following the intravenous administration of hexamethonium in supine subjects is accompanied by no or a slight reduction of cardiac output (Fick) and by a significant decrease in total peripheral resistance. The changes in local vascular resistances vary markedly with the vascular area under study. Thus, muscle blood flow (calf segment) usually decreases, whereas foot and digit flows increase many fold. Effective hepatic-portal blood flow usually does not change significantly indicating a decrease in peripheral resistance, while renal blood flow decreases initially and then slowly returns toward control levels. In the early hypotensive period there is a decrease in renal plasma flow, glomerular filtration rate and filtration fraction accompanied by moderate oliguria. The oliguria is not as marked nor the return of clearance values to the baseline as rapid as after veratrum viride.

These studies suggest that the autonomic blockade following hexamethonium does not produce a uniform decrease in peripheral vascular resistance. In the presence of a generalized inhibition of the sympathetic nervous system the percentage changes in peripheral resistance vary greatly in different vascular areas. It is suggested on the basis of this and other evidence that the sympathetic vasoconstrictor nerves are of great importance in maintaining vascular tone in certain regions such as the hand and feet and of much less importance in other vascular areas such as the kidneys and muscles.

The Cholate Mechanism and Its Role in the Production of Hypercholesteremia Found in Clinical and Experimental Nephrosis. Meyer Friedman,* Sanford O. Byers and Ray H. Rosenman, San Francisco, California.

Nephrotic patients having hypercholesteremia, similar to other types of hypercholesteremic patients, were found to have an increase in their plasma cholate content. Thus, in fifteen nephrotic patients, the average plasma cholesterol was $695 \mathrm{mg} . / 100 \mathrm{cc}$., and the plasma cholate was $19.2 \mathrm{mg} . / 100 \mathrm{cc}$. The average plasma cholesterol and cholate concentration in twenty-five normal subjects was 228 and $5.3 \mathrm{mg} . / 100 \mathrm{cc}$. respectively. These findings were 
of interest because elevation of cholate in animals has been found by us to induce hypercholesteremia.

In order to study this relationship further, nephrosis was induced in rats. Plasma cholate rose in ten rats from 6.2 to $26.2 \mathrm{mg} . / 100 \mathrm{cc}$., one week after injection of anti-kidney serum. Plasma cholesterol rose from 64.3 to $279.6 \mathrm{mg} . / 100 \mathrm{cc}$. Since a similar degree of "hypercholatemia" induced in a normal rat by intravenous injection of cholate leads to a cholesterol rise comparable to that found above, it would appear that the "hypercholatemia" observed in nephrotic rats is the cause of their hypercholesteremia.

The oral administration of $100 \mathrm{mg}$. of cholate/day for 3 days to a series of nephrotic rats also demonstrated this mechanism. The plasma cholate of cholate-fed nephrotic rats was three times higher than that of the control nephrotic rats. The plasma cholesterol concentration also changed in the same direction, being twice as high in the cholate fed series as in the control series.

The discovery of a "hypercholatemia" in both patient and animal having nephrotic hypercholesteremia, together with evidence suggesting that the cholesterol rise in the animal is due to this "hypercholatemia," adds support to the hypothesis that clinical and experimental states of hypercholesteremia are dependent in some manner upon blood cholate content.

\section{Delayed Cutaneous Hypersensitivity in Sarcoidosis.} George J. Friou, Newington, Conn. (Introduced by Robert H. Green).

Delayed intracutaneous reactions to oidiomycin, trichophytin, and mumps antigens were studied in twenty-two patients with proven sarcoidosis and sixty-four controls. Fifteen cases of sarcoidosis and twenty controls were tuberculin negative. The antigens used, to which most individuals react, are immunologically distinct from each other and from tuberculin.

Positive tests were less frequent and generally smaller in the sarcoidosis group than among the controls, and this was particularly true of tuberculin negative sarcoidosis. Sarcoidosis cases with negative tuberculin reactions had a significantly smaller mean number of positive tests per case than tuberculin negative controls or tuberculin positive sarcoidosis. There was no appreciable difference between tuberculin positive sarcoidosis and corresponding controls. Five cases of sarcoidosis and no controls failed to react to any antigens.

Considered individually, oidiomycin and mumps antigens gave significantly less frequent positive tests in tuberculin negative sarcoidosis than in corresponding controls. The mean diameters of positive oidiomycin reactions in these groups differed significantly in the same direction. In individuals in these groups with a history of mumps the same difference was observed with mumps antigen. For trichophytin the difference between these groups was just significant when mean diameters for all cases tested were compared.

It was concluded that the decrease in delayed hypersensitive reactions in sarcoidosis is not limited to tuber- culin, but is a broad, immunologically non-specific phenomenon probably affecting, when present, all delayed cutaneous hypersensitive reactions. These results remove some support from the theory that sarcoidosis is of tuberculous etiology, since it is usually assumed that the decreased reaction is specific for tuberculin. They indicate that delayed cutaneous reactions are not reliable in specific etiologic studies of sarcoidosis. With this simple technique it is possible to evaluate more completely the significance of a negative tuberculin reaction in this and other conditions.

The Circulatory Consequences of Pneumoperitoneum in Pulmonary Emphysema. RoBert H. Furman, Thomas M. Blake and Mildred T. Stahlman, Nashville, Tennessee. (Introduced by Hugh J. Morgan).

An increasing experience with pneumoperitoneum has suggested that benefit may accrue the patient which is difficult to assess by common pulmonary function tests. Utilizing cardiac catheterization, seven severely emphysematous subjects with low immobile diaphragms were studied at rest and after exercise. Resting output values were low and showed variable but slight change after pneumoperitoneum. Exercise before pneumoperitoneum produced a fall in cardiac output in five, no change in one and a rise in another. No fall was noted after exercise after treatment. Two subjects whose outputs fell after exercise before pneumoperitoneum showed no change after exercise after treatment; output rose in five. Exercise in every instance evoked ,maximal ventilatory responses. The post-pneumoperitoneum improvement in the cardiodynamic response to exercise was associated with an increased oxygen consumption and extraction and smaller A-V oxygen difference than that noted following exercise before treatment. Increased intrapleural-intraperitoneal pressure differentials and probably improved intrapulmonary gas mixing appear to be the responsible mechanisms. The same mechanisms may obtain in the familiar squatting posture seen after exercise in patients with Tetralogy of Fallot.

Effect of Thyrotropin (TSH) on Adreno-Cortical Function. J. Lester Gabrilove, New York, N. Y. (Introduced by Louis J. Soffer).

Thyrotropin (TSH) was administered to three groups: 1) primary hypothyroidism, 2) hypopituitarism (Sheehan's Syndrome), and 3) euthyroidism. Studies included determination of the basal metabolic rate, the serum protein bound iodine level (SPI), the urinary excretion of the neutral 17-ketosteroids and 11 oxygenated corticoids, the circulating eosinophile level, exophthalmometric readings, and serial skin biopsy. Patients with primary myxedema to whom TSH was given failed to demonstrate any clinical improvement nor was there noted any alteration in the BMR, SPI, or urinary steroid excretion. Following the administration of TSH to the patients with Sheehan's syndrome, there was noted clinical and laboratory improvement of the hypothyroidism, concomitant with a decrease in the urinary excretion of the 11 
oxygenated compounds and the precipitation of adrenal insufficiency. The urinary excretion of the neutral 17ketosteroids remained unaltered. In one euthyroid subject, the administration of TSH was followed by a rise in the BMR and SPI and a slight decrease in the urinary excretion of the 11 oxygenated compounds. The urinary excretion of the neutral 17-ketosteroids remained unchanged.

In the skin of patients with Sheehan's syndrome, there was a deposition of metachromatic staining material presumably mucoprotein in nature, similar to that observed in patients with primary hypothyroidism. This substance differed from that encountered in normal skin in its more intense staining characteristics and its localization. The administration of TSH to patients with Sheehan's syndrome or the administration of thyroid extract to subjects with primary hypothyroidism resulted in a decrease in the skin content of the mucoprotein-like material.

Effect of Aureomycin Upon Nitrogen Balance and Urinary Riboflavin Excretion in Undernourished Men. George J. Gabuzda, George G. Jackson and MarGARET E. Grigsby, Boston, Massachusetts. (Introduced by Maxwell Finland).

Aureomycin was administered to three undernourished men maintained on constant diets providing $75 \mathrm{gm}$. of protein and 2800 calories daily. Each patient was studied during three periods: initial control (6 to 9 days), aureomycin administration (2.5 to $3.0 \mathrm{gm}$. orally daily for 9 to 12 days), final control ( 9 to 12 days). None of these patients developed diarrhea, stomatitis or glossitis. Anorexia occurred but constant food intakes were maintained.

During the initial control period and the first four days of aureomycin two patients gained and one maintained weight. All patients lost weight during the latter part of the aureomycin period and the first several days of the final study period. Thereafter all patients regained weight.

Average daily urinary nitrogen excretions during the initial control periods were $6.6,7.1$, and $5.5 \mathrm{gm}$., and nitrogen balances $+4.7,+3.8$, and $+5.5 \mathrm{gm}$. daily in the three patients, respectively. Aureomycin administration was followed after 1 to 6 days by increases in urinary nitrogen. Average daily nitrogen excretions during aureomycin were $8.5,10.9$, and $7.1 \mathrm{gm}$., and nitrogen balances $+2.3,-0.2$, and $+3.7 \mathrm{gm}$. daily, respectively. Urine nitrogen excretions returned to initial levels during the final control period. Stool nitrogen excretions were not significantly altered during these studies.

Urinary riboflavin (microbiologic assay) increased 2.5-fold during aureomycin administration, and preceded the increases in urinary nitrogen by 1 to 5 days. In the final study period riboflavin excretion first decreased to less than the initial control value and then gradually increased again almost to control levels. Stool riboflavin excretion decreased during aureomycin.

In vitro, aureomycin does not invalidate the microbiologic assay for riboflavin.
Serial stool cultures revealed no consistent quantitative or qualitative alterations.

Conclusion: Administration of aureomycin to three patients was associated with loss of weight and an increase in urinary nitrogen and riboflavin excretions.

\section{Changes Suggesting Emphysema in Physiologic Respira- tory Measurements in Patients with Neuromuscular Disorders, and in Healthy Individuals. MORTON GALD- ston, New York, N. Y. (Introduced by J. Murray Steele).}

Patients suffering from atrophy of thoracic, pectoral and abdominal muscles as a result of neuromuscular disease were found to exhibit some disturbances in physiologic measurements commonly noted in instances of chronic obstructive pulmonary emphysema. These are pulmonary distension (absolute and relative increase in residual air volume), decreased vital capacity and maximal breathing capacity and an abnormally large respiratory dead space volume. Certain seemingly healthy subjects studied over a period of several years have manifested similar physiologic disturbances except for decreased maximal breathing capacity. Some of the apparently healthy subjects also exhibit an increased portion of mixed venous blood which fails to be oxygenated in the lungs and a decreased alveolar diffusing capacity for oxygen. All of these disturbances together are present in patients suffering from chronic emphysema.

The physiologic alterations in emphysema are believed to reflect loss of pulmonary elasticity and destruction of parenchymal tissue due to chronic cough and bronchial obstruction. The fact that none of the patients studied had a history of respiratory complaints or exhibited any of the physical or roentgenographical signs of emphysema makes it doubtful that they have what is generally considered to be emphysema. The evidence suggests that disease of respiratory muscles can bring about pulmonary distension. Even in seemingly healthy individuals unrecognized impairment of action of respiratory muscles or lack of mobility of the thoracic cage can give rise to similar changes. The presence of such unsuspected physiologic derangements in certain individuals may account for instances of seemingly rapid development of pulmonary insufficiency following respiratory infections.

\section{Effect of Combined Cortisone and Penicillin Therapy on Experimental Streptococcal Pneumonia. RoBerT J. Glaser, and Lenore H. Loeb, St. Louis, Missouri.} (Introduced by Carl G. Harford).

Recent studies from this laboratory have demonstrated that the course of experimental hemolytic streptococcal pneumonia is more fulminating in rats pretreated with cortisone than in untreated control animals. On the other hand, in a number of human diseases, including several of bacterial origin, cortisone exerts a favorable effect on some signs and symptoms even though the basic pathologic process may be unaffected.

The latter observation raises the question as to whether cortisone might constitute a useful adjunct in the treat- 
ment of constitutional manifestations of certain infections for which effective antibacterial agents are available. In order to obtain evidence bearing on this point, the effect of combined penicillin and cortisone therapy in experimental streptococcal pneumonia in rats was studied.

Various experiments were performed in which rats were inoculated by the intrabronchial route with hemolytic streptococci. One group of animals was given cortisone for five days prior to inoculation and subsequently treated with penicillin. Control rats received no cortisone but were given identical courses of penicillin. The cortisone group exhibited less favorable survival rates and increased numbers of purulent complications in comparison with the control group.

When, however, treatment with cortisone and penicillin was begun after inoculation, the infections appeared to be, if anything, less severe than those in control animals receiving penicillin alone. The lesions observed in systematic histologic study of the lungs of rats receiving combined therapy were less intense than those seen in the lungs of the penicillin controls.

Since cortisone modifies the inflammatory response to bacterial infection, in those infections for which effective chemotherapy is available to control growth of organisms per se, the use of cortisone to limit inflammation and the concomitant toxic manifestations, which are often severe, may be of value. The results reported herewith suggest that such a possibility merits consideration.

\section{Atrio-Septo-Pexy: A New Surgical Treatment for Atrial Septal Defect. Hemodynamic Studies in a Case Before and After Surgery. Harry Goldgerg, Charles P. Bailey, George D. Geckeler and William Likoff, Philadelphia, Pennsylvania. (Introduced by Joseph $\mathbf{R}$. DiPalma).}

A new surgical treatment for atrial septal defect is presented. The chest is opened by a right 4th interspace incision. Following opening of the pericardial sac, a purse-string suture is placed about the right atrial appendage. Incision is made in the appendage and the left ungloved forefinger introduced into the right atrium. The finger is advanced into the chamber and through the defect. With the finger in-situ the right atrial appendage is sewn to the edge of the defect. By this means the residual cavity of the right atrium is gradually rendered no longer in communication with the left. Following the atrio-septo-pexy, the right atrial appendage is ligated.

Hemodynamic studies were made in a case of interatrial defect before and after the above operation. A 38 year old woman with a murmur since birth presented dypsnea, cardiac enlargement, rough systolic murmur in third left intercostal space, accentuated 2nd pulmonic sound, enlarged pulmonary arteries, hilar dance and increased vascular markings. The ECG showed right heart strain. During cardiac catheterization, the catheter was passed through an interatrial defect into the left atrium. The pulmonic blood flow was $17.31 / \mathrm{m}$; systemic blood flow $4.1 \mathrm{1} / \mathrm{m}$ indicating a left to right shunt of $13.01 / \mathrm{m}$. The pulmonary arterial pressure was $90 / 40 \mathrm{~mm} \mathrm{Hg}$ and brachial arterial oxygen saturation $94 \%$. Following sur- gery, the systolic murmur was inaudible. Though the pulmonary arteries were still enlarged, the hilar dance was less marked.

Recatheterization, 17 days post-operatively, revealed: Pulmonic and systemic blood flows $5.91 / \mathrm{m}$; pulmonary arterial pressure $48 / 33 \mathrm{~mm} \mathrm{Hg}$; brachial arterial oxygen saturation $82 \%$. The latter was thought to be due to pulmonary factors subsequent to surgery rather than to a right to left shunt. The surgical procedure eliminated the left to right shunt in the atrium and reduced the pulmonary arterial pressure by $30 \%$.

The Effects of Cortisone on the Reaction to Bacterial Endotoxins and Infections: Imitation by Agents Which "Blockade" the R. E. System. RoBert A. Good, Floyd W. Denny, Jr. and Lewis Thomas, Minneapolis, Minnesota.

Previous studies showed that a single injection of gramnegative bacterial toxin causes bilateral renal cortical necrosis in cortisone-treated rabbits. This lesion can only be produced in normal rabbits by giving two intravenous injections of toxin spaced 24 hours apart (the generalized Shwartzman reaction). The observation suggested that a defense mechanism which protects normal rabbits against toxin is impaired by cortisone treatment. In view of the known capacity of cortisone to cause dissolution of lymphoid tissues, the possibility that defense might involve cells of the reticuloendothelial system was considered.

Beeson showed that R. E. "blockade" with thorotrast or trypan blue enhances susceptibility to the local Shwartzman reaction. Experiments were undertaken to determine whether these substances have an effect comparable to that of cortisone on the response to one injection of toxin.

A single injection of meningococcal or $S$. marcescens toxin, 6 hours after an injection of thorotrast, regularly produced the following results: 1) The lethal effect of toxin was enhanced 500-fold. 2) Bilateral cortical necrosis of the kidneys occurred in a high percentage. 3) An intradermal injection of toxin caused local skin hemorrhage as well as renal cortical necrosis.

Identical results were obtained when trypan blue was used for "blockade." Neither thorotrast nor trypan blue caused illness or tissue lesions when given alone, or when given a few hours after, instead of before, toxin.

The previously demonstrated capacity of cortisone to enhance streptococcal infection was similarly imitated by thorotrast. When streptococci were injected a few hours after thorotrast, extensive and uniformly fatal septicemia occurred.

The results suggest that interference with a protective function of the R. E. system may be the basis for the effects of cortisone on bacterial infection and intoxication.

The Effect of Glucose Diuresis on the Renal Excretion of Sodium Bicarbonate. A. V. N. Goodyer, L. G. WeLt, J. H. Darragh, W. A. Abele and W. H. Meroney, New Haven, Conn. (Introduced by Arthur J. Geiger).

The urinary excretion of bicarbonate is thought to be the result of partial rejection, in the distal tubules, of that 
small (about 15\%), constant fraction of filtered bicarbonate which escapes reabsorption in the proximal tubules. Any increase in this fraction would augment the urinary excretion of bicarbonate, provided distal tubular activity remained constant.

It was therefore of interest to determine whether glucose diuresis (probably mediated in the proximal tubules) would alter the rate of excretion of bicarbonate when distal tubular reabsorption of bicarbonate was minimal.

A high rate of bicarbonate excretion was induced in three hydropenic normal subjects by the steady infusion of a $1.4 \%$ solution of sodium bicarbonate. When urine flow and the excretion of bicarbonate and chloride were constant, $750-1000 \mathrm{cc}$. of a $25 \%$ solution of glucose was administered.

Urine flow and the excretion of sodium chloride increased greatly as glycosuria supervened. Nevertheless, the excretion of bicarbonate decreased as much as $50 \%$. The excretion of potassium increased from low values in one experiment, and decreased from high values in two. Glomerular filtration was unchanged or increased slightly.

The decrease in bicarbonate excretion was correlated with the dilution of serum bicarbonate, chloride and sodium by water withdrawn from cells as a result of the hyperglycemia. Serum $\mathrm{pH}$ also decreased slightly.

The data are interpreted to indicate that a decrease in serum bicarbonate (or $\mathrm{pH}$ ) may be the stimulus that increases the tubular reabsorption of bicarbonate in spite of a large proximal tubular diuresis.

The data emphasize the priority of influences originating in the internal environment (concentration of bicarbonate or $\mathrm{pH}$ of serum) over local renal factors (proximal tubular diuresis) in the regulation of the rate of excretion of bicarbonate.

The Importance of a 'Sodium Retaining Factor' in the Urine in the Mechanism of Edema Formation. EDGAR S. Gordon,* Jerome J. Chart and Elva S. Meyer, Madison, Wisconsin.

Presence in the urine of an antidiuretic factor, possibly posterior lobe pituitary hormone, has been demonstrated in various types of clinical conditions involving fluid accumulation. The cause for and the mechanism of this phenomenon is unexplained. Using a new, sensitive biological assay method for a sodium retaining factor in urine, high titers of this material have been found consistently in clinical patients exhibiting edema and fluid retention. There is striking correlation between these titers and fluid and sodium balance. Toxemia of late pregnancy, cardiac decompensation, renal disease with edema and hepatic cirrhosis have been studied. The factor under study is believed to be steroidal in nature and probably of adrenal origin. A working hypothesis has been developed for the mechanism of abnormal fluid accumulation from various causes in which altered adrenal function with sodium retention is believed to be primary, followed by increased antidiuretic hormone formation.
Studies of Pulmonary Distention. Frank D. Gray, JR.* and Frieda G. Gray, New Haven, Connecticut.

The functional residual capacity of the lung (FRC), a measure of the total lung volume at the end of a normal expiration, reflects the degree of expansion or distention of the resting lung. In general, factors tending to impair passive expiration increase the distention of the resting lung and diminish alveolar ventilation.

In an effort to determine some of the factors influencing pulmonary distention studies were undertaken of the FRC as affected by various therapeutic measures including vaponefrin inhalation, digitalization of patients with chronic pulmonary disease or mitral stenosis, and administration of cortisone or ACTH to patients with rheumatoid arthritis. The last group had, in some cases, evidence of spondylitis. Finally, vaponefrin and cortisone therapy were studied in a patient with Boeck's sarcoid.

As was expected vaponefrin reduced the FRC in proportion to the degree of bronchospasm present. In patients with chronic pulmonary disease, digitalization produced an increase in FRC in some and a decrease in others with no discernible pattern. In the mitral stenosis group digitalis regularly increased the FRC. The increase was roughly related to the degree of pulmonary artery hypertension developing after digitalization. Cortisone or ACTH in rheumatoid arthritis patients resulted in a dramatic and consistent decrease in FRC paralleling the increase in skeletal mobility. A patient with severe Boeck's sarcoid treated with cortisone showed a marked increase in FRC followed by a decrease toward normal. An initial increase was also seen following vaponefrin. In this case the drugs may have initially opened collateral respiratory channels thereby overcoming atelectasis, then relieved bronchial obstruction thus finally decreasing distention.

It was concluded that bronchial obstruction, pulmonary vascular engorgement, and immobility of the chest cage may produce reversible pulmonary distention.

The Stomach as an Endocrine-like Organ. Factors Infuencing Urinary Uropepsin Excretion. SEYMoUR J. Gray, John A. Benson, Jr., Robert W. Reifenstein and Colin RAMSEy, Boston, Mass. (Introduced by Clifford L. Derick).

Uropepsin is a proteolytic enzyme present in the urine of healthy persons in constant amounts. Its origin in the stomach has been established by the disappearance of the enzyme following total gastrectomy. The urinary level of uropepsin is considered a measure of the endocrine-like activity of the stomach resulting from the secretion of pepsinogen into the blood stream by the peptic gland.

Studies were undertaken to investigate the role of the stomach as part of a hypothalamic-pituitary-adrenal-gastric pathway. ACTH and cortisone administered over a period of 3 to 4 weeks produced a marked increase approximating $200 \%$ in the basal and nocturnal gastric secretion of acid and pepsin and a similar increase in urinary unropepsin excretion. In every instance adrenocorticotropic hormone increased the acid secretion and uropepsin excretion to the active duodenal ulcer level. 
The adrenal steroids including Compound $F$ produced a uniform and standardized increase in uropepsin excretion approximating $150-200 \%$ in normal subjects within $24-48$ hours. This hormonal response appears to be independent of vagal control and parallels the circulating eosinophiles in the blood. Similar results have been obtained in patients after vagus section.

The urinary uropepsin excretion in patients with Cushing's disease (with adrenal hyper-function) and in patients with brain tumors involving the hypothalamus was elevated significantly. Diminished levels were observed in patients with Addison's disease and hypopituitarism. Exceedingly low levels were found in 80 patients with pernicious anemia.

The stress of surgical procedures and burns appears to produce identical increases in the uropepsin excretion parelleling the fall in circulating eosinophiles. Other stress factors such as physical exertion, anoxia, shock and $x$-ray irradiation elicit similar responses.

The relationship of hormonal influences to the gastric responses to stress as part of a hypothalamic-pituitaryadrenal mechanism will be discussed.

Permeability Studies on the Blood Cerebrospinal Fluid Barrier in Polyneuritis. MARK A. GrIfrIN, Philadelphia, Pennsylvania. (Introduced by George D. Gammon).

The penetration of the dye, fluorescein, into the spinal fluid after intravenous injection has been studied in cases of polyneuritis in which the cerebrospinal fluid protein is high. Normally this dye cannot be detected in spinal fluid after intravenous injection but in the neuritis cases showing a protein of $100 \mathrm{mg}$. per $\mathrm{ml}$. the dye was readily detectable in amounts roughly proportional to the protein level. This demonstrates an increased penetration of this substance and suggests that some at least of the protein may be derived from this serum through increased permeability of meningeal and vascular surfaces. This concept, if correct, might explain certain of the clinical features of the disease.

Hexamethonium Salts Alone or with 1-Hydrozaline or Regitine for Patients with Hypertension. K. S. GRIMson,* Durham, N. C.

Tests in animals and man reveal that hexamethonium bromide, chloride, bitartrate, and citrate administered parenterally produce hypotension with minimum tachycardia, interrupt vaso-motor reflexes and delay transit time of barium; actions resembling those of the ganglionic blocking agent, Etamon. Single oral doses of one gram of the bromide, chloride, or bitartrate produce minimal effects. However, during the first several days evidence of effectiveness developed in each of 60 patients now continuing treatment, two to six grams daily, as long as 17 months. Most patients have relief of symptoms, some have moderate and a few marked reduction of blood pressure.

1-Hydrozaline, Apresoline, initially tested and given to patients in 1948 occasionally reduced blood pressure when
$100 \mathrm{mg}$. were given intravenously or as much as $250 \mathrm{mg}$. orally. Continuing oral treatment was discontinued because of little reduction of pressure and occurrence of side effects. Regitine, an imidazoline derivitive, was tested in 1949 using 60 or $120 \mathrm{mg}$. doses orally. It produced more reduction of pressure but continuing treatment was discontinued because of tachycardia. Apresoline or Regitine has now been given to those of the 60 patients taking hexamethonium daily, whose blood pressure has not been satisfactorily reduced. Some additional reduction of blood pressure occurred. Tachycardia has been less frequently troublesome. Of 7 of these patients now tested by intravenous injections of $50 \mathrm{mg}$. of Regitine slight increase of pulse rate occurred in 5 and typical marked increase in only two.

\section{The Renal Excretion and Extrarenal Uptake of Histidine} and of Total Amino Acids in Man, and the Effect of ACTH and Cortisone Administration. David GroB, Baltimore, Md. (Introduced by F. B. Bang).

The factors responsible for the increased histidinuria induced by ACTH or cortisone administration were investigated by study of the mechanism of renal excretion of endogenous and injected histidine, the renal and extrarenal components in the disappearance of injected histidine, and the influence of ACTH and cortisone on these processes. Histidine was found to be normally excreted by a mechanism involving tubular reabsorption of approximately 93 per cent of filtered histidine, with no limitation of transfer capacity in the range of tubular loads studied. The rate at which the injected compound disappeared from the extracellular fluid by means other than renal excretion was 10 to 30 times the rate of disappearance through the kidneys. The administration of ACTH or cortisone produced a three-fold increase in the rate of urinary excretion of endogenous or injected histidine, which was attributable primarily to a decrease in tubular reabsorption to approximately 80 per cent of filtered compound. Increased glomerular filtration played a lesser role. The rate of extrarenal uptake of injected histidine was reduced by 30 to 50 per cent during hormone administration, but this could account for only part of the increased excretion. It is suggested that the diminished extrarenal and tubular transport of histidine induced by ACTH or cortisone may reflect a widespread decrease in the cellular transport of this compound.

The increased excretion of total amino-acid nitrogen induced by ACTH or cortisone was also attributable in large part to decreased tubular reabsorption. The data indicate that part of the increased total nitrogen excretion and negative nitrogen balance induced by these hormones is renal in origin.

Protein Synthesis by Human Colon Mucosa. CharLes M. Grossman, James D. Hauschildt and James D. Case, Portland, Oregon. (Introduced by Arthur W. Frisch).

There is abundant evidence that in-vitro incorporation of radioactive amino acids into protein reflects protein 
synthesis. We have found sigmoid colon mucosa to incorporate significant amounts of carboxyl-labeled glycine into its protein. Colon mucosa strips from surgical resections were washed and incubated with 0.33 microcuries of labeled glycine. Proteins were precipitated, lipid extracted, and radioactivity measured. Controls were obtained by precipitating at zero time. Results are expressed as specific activities (S. A.-counts per minute per milligram dry protein).

S. A. of colon protein ranged from 65 to 1175 . Zero time mean $\pm \mathrm{S}$. $\mathrm{E}$. was $6.3 \pm 1.3$. Incorporation was regularly depressed by penicillin but not by aureomycin. Inhibition by nitrogen or cyanide was marked. Omitting calcium or magnesium from the buffer gave inconsistent results in contrast to consistent depression with liver slices. Homogenized tissue incorporated insignificant amounts of labeled glycine, yet good bacterial growth was demonstrable. Slices of colon smooth muscle yielded protein with S. A. 45 , compared to 350 with mucosa from the same patient. Mucoprotein was isolated and found to have significant S. A., although invariably lower than total protein from the same tissue. $S^{25}$ inorganic sulfate was found to be incorporated into both total protein and mucoprotein. The $\mathrm{S}^{26}$ was easily removed from total protein by solution in alkaline urea and reprecipitation, whereas the $\mathrm{C}^{14}$ label was not removed by this procedure. This may be explained by coprecipitation of mucoprotein with total protein.

These findings indicate that the distal colon mucosa is an active membrane participating in metabolic functions other than absorption and storage. The data suggest that in addition to synthesizing mucoprotein the cells are capable of incorporating labeled glycine into other protein moieties.

The Effects of Subtotal Adrenalectomy Combined with Sympathectomy Upon the Cerebral Vascular Resistance of Patients with Severe Hypertension. JoSEPH $\mathrm{H}$. Hafkenschiel and Carl K. Friedland, Philadelphia, Penna. (Introduction by William A. Jeffers).

Blood flow to the brain was measured by the nitrous oxide method in six patients before, and at intervals ranging from three to fifteen months after operation. Mean changes from the preoperative to the initial postoperative study were: Mean arterial pressure 174 to 114 $\mathrm{mm}$. $\mathrm{Hg}$, cerebral vascular resistance 3.7 to $2.1 \mathrm{~mm}$. $\mathrm{Hg}$ per cc. blood per 100 grams brain per min., cerebral blood flow 50 to $63 \mathrm{cc}$. per 100 grams per min., cerebral oxygen uptake 3.8 to 4.1 cc. per 100 grams per min., arterial carbon dioxide tension 42 to $39 \mathrm{~mm}$. $\mathrm{Hg}$, jugular venous oxygen tension 32 to $37 \mathrm{~mm}$. Hg. The last is assumed to represent minimal cerebral oxygen tension. The only significant changes were the reductions in blood pressure and cerebral vascular resistance. These were highly significant $(p<0.01)$. Five of six patients showed cerebral vascular resistances above the mean $(2.8 \pm 0.7)$ for a large series of hypertensive patients. After adrenalectomy, four of the six had resistance values below that of Kety's normals $(1.6 \pm 0.4)$, the other two having decreased from 4.6 to 3.9 and from 3.7 to 2.7 respectively.
Three patients were tested again three to thirteen months after the first post adrenalectomy study. The time studied after adrenalectomy did not appear to influence the degree of resistance change. These observations suggest that the increased cerebral vascular tone of these patients has been reduced by sympatho-adrenal surgery without alterations in cerebral blood flow, oxygen uptake or tension.

Observations on Body Composition and Body Water Metabolism in Normal Pregnancy. Harold B. Haley and JoHN W. WoODBURY, Boston, Massachusetts. (Introduced by C. Sidney Burwell).

Deuterium was used to measure total body water in pregnant women.

Equilibrium time after injection of $\mathrm{D}_{2} \mathrm{O}$ was the same in three patients in midpregnancy as in non-pregnant women. Cord blood, placental tissue, colostrum and milk had the same deuterium concentration as blood serum.

Total body water was determined in three women at four to six-week intervals during the last two-thirds of pregnancy. Each patient gained weight, water, and solids. In the three weeks before delivery, the average gain of water was 1.5 liters (range 0.9-2.1) ; of weight, 0.9 kilograms (range $0-1.5$ ). This difference indicates differential accumulation of water over solids.

Body water determinations were made in five patients during the first twelve hours post-delivery and one week later. All lost body solids. (Average loss 1.3 kilograms; range 0.9-2.0). Four lost both weight and water; one gained weight by accumulating water. The loss of solids is interpreted as due to (1) lochia, (2) involution of hypertrophied organs, (3) postpartum nitrogen loss possibly analogous to the metabolic response to trauma.

The disappearance rate of $\mathrm{D}_{2} \mathrm{O}$ from the blood, and therefore from the body water, measures the water turnover rate or the balance between total intake and total output. The three patients studied before delivery had a normal turnover rate during the prenatal period. Of six patients studied in the first week postpartum, five showed a high water turnover rate with an abrupt change to lower levels at about the fourth postpartum day.

Thus at the end of normal pregnancy, these women selectively retained water. In the immediate postpartum period, there was an increase in water turnover rate and these rates fell to normal about the fourth day. In the first postpartum week there was a loss of body solids and in most patients a net loss of water.

\section{Studies in the Mode of Action of Chemotherapeutic Drugs in Bacterial Endocarditis. MORTON HAMBURGER * and Leon Stein, Cincinnati, Ohio.}

The success of penicillin in the treatment of subacute bacterial endocarditis, in contrast with the ineffectiveness of sulfonamides has often been attributed to ability of the former and inability of the latter to penetrate fibrin vegetations. The indifferent success of the "broad spectrum" antibiotics has been ascribed to their bacteriostatic rather than bactericidal action. 
In an attempt to throw light upon these hypotheses, experiments of Liebold and Nathanson were modified and extended, using a strain of streptococcus viridans from a case of endocarditis, and employing penicillin $G$, sodium sulfathiazole, aureomycin, terramycin, and chloramphenicol as test drugs. The streptococcus was seeded in fibrin clots prepared from human Fraction I, in petri dishes, and in brain heart infusion agar. Filter paper discs saturated with different concentrations of the test drugs were placed upon the surface, following which they were incubated at $37 \mathrm{C}$ for 24 hours.

The results were as follows:

(1) Zones produced by all test drugs, over a wide range of concentrations, were usually wider on fibrin plates than on agar plates.

(2) Colonies were seen under low power magnification in the microscopic clear zone produced by sulfathiazole and broad spectrum antibiotics, but not in penicillin zones.

(3) Cultures from the surface of penicillin zones were sterile in all concentrations tested. Cultures from the depths of high concentration penicillin zones were negative after 1 week's incubation; from moderate concentration zones negative at 24 hours but positive in 48 ; from low concentration zones positive after over-night incubation. In contrast, culture from the depths of all broad spectrum antibiotic and sulfathiazole zones were positive, and even from the surface were negative in only the highest concentrations emplojed.

The experiments suggest that the bactericidal action of penicillin in the depths of a vegetation is probably its most important characteristic in determining its effectiveness in bacterial endocarditis.

A Hemolytic Mechanism in Pernicious Anemia Influenced by $B_{12 .}$. Henry E. Hamilton, Raymond F. Sheets and Cuinton D. Janney, Iowa City, Iowa. (Introduced by Elmer L. DeGowin).

Eight patients with pernicious anemia were transfused with fresh blood from normal donors of heterologous, compatible $\mathrm{A}-\mathrm{B}-\mathrm{O}$ group and $\mathrm{Rh}$ type. The concentrations of donors' erythrocytes during periods up to 120 days were measured by serial counts of inagglutinable cells by a modification of Ashby's technique yielding accurate data for mathematical analysis.

Analysis of the disappearance curves of normal transfused cells showed normal survival with $B_{12}$ started 9 and 12 days before transfusion. In patients beginning treatment 9 days before and 1,4 , and 5 days after transfusion, random destruction of the normal erythrocytes, irrespective of age, was demonstrated. In general, the rate of loss increased the later $B_{12}$ was given, with the fastest rate in the untreated patient.

In six studies the rate of random cell destruction was constant throughout the four-month period, despite coincidentally adequate reticulocyte responses and satisfactory rises in total erythrocyte counts. In two studies exhibiting the fastest rate of destruction the rate accelerated about 70 days after transfusion.

Conclusions: (1) Fresh normal erythrocytes are so damaged in a few days in the milieu of the human body deficient in $B_{12}$ that they are destroyed in random fashion, presumably by the trauma of circulation. (2) The cell damage incurred in the first few days is not reversible by the subsequent administration of $B_{12}$. (3) When severe grades of erythrocyte damage have occurred, the chemical changes of aging or the trauma of 70 days of circulation further hasten destruction. (4) These studies indicate that 45 micrograms of $B_{12}$ weekly must be present in the body for at least 10 days before a safe environment is provided for normal erythocytes. Until further evidence is available, it is assumed that the hemolytic mechanism exerts similar effects on the circulating erythrocytes of the patient.

Biosynthesis of Nucleic Acids Studied in Unicellular Systems. Leonard Hamilton, George Bosworth Brown and C. Chester Stock, New York, New York. (Introduced by David Karnofsky).

As a biological model for studying polynucleotide synthesis in a single species of mammalian cells (such as human leukocytes) as distinct from overall synthesis in intact mammals, the incorporation of $\mathrm{C}^{14}$-labelled purines into the nucleic acids of Paramecium aurelia was studied. Both "killer" (51-7K) and "sensitive" (51-75B) varieties of $P$. aurelia utilize adenine-8- $C^{14}$ for the synthesis of nucleic acid adenine and guanine; the nucleic acid adenine and guanine show the same proportionate uptake of $\mathrm{C}^{\mathbf{1 4}}$; 2,6-diaminopurine-2- $\mathrm{C}^{14}$ is converted into nucleic acid guanine and adenine but preferentially to the former. The purine utilization patterns differ from those of Aerobacter aerogenes on which paramecium feeds. $A$. aerogenes utilizes adenine for the synthesis of polynucleotide adenine and guanine with preferential transformation to the former, 2,6-diaminopurine and to a greater extent guanine-8- $\mathrm{C}^{\mathbf{1 4}}$ are used preferentially for the formation of polynucleotide guanine and comparatively little for transformation into polynucleotide adenine. Another protozoan, the chrysomonad flagellate Ochromonas, incorporates adenine twice as readily into polynucleotide adenine as into guanine; it utilizes guanine and 2,6-diaminopurine for both polynucleotide adenine and guanine with preferential incorporation into the guanine. These patterns of purine incorporation can be compared with these published for other species: $L$. caseii in which adenine and guanine serve equally well as precursors of polynucleotide adenine and guanine; the rat in which adenine is utilized for polynucleotide adenine and transformed into guanine but in which guanine is only slightly utilized; Tetrahymena geleii in which adenine is utilized for polynucleotide adenine but not transformed into guanine whereas guanine is utilized for both polynucleotide guanine and adenine. Similar studies are now in progress on the biosynthesis of nucleic acids in normal and leukemic human leukocytes.

\footnotetext{
Effects of Restriction of Dietary Fat and Cholesterol Upon Serum Lipid Patterns in Patients with Hypertension. Frederick T. Hatch and Forrest E. KenDALl, New York City, New York. (Introduced by David Seegal).
} 
Serum lipid patterns, including free and total cholesterol, lipid phosphorus and neutral fat have been determined in hospitalized patients with severe hypertension on four levels of dietary fat and cholesterol intake. Forty patients were studied on a normal fat ( $85 \mathrm{gm}$. per day) and cholesterol (1 gm. per day) intake and then on a cholesterol-free diet containing $3 \mathrm{gm}$. of fat per day (Kempner rice diet). Groups of these patients were also studied on fat intake levels of 50 to $60 \mathrm{gm}$. per day (12 patients) and 20 to $40 \mathrm{gm}$. per day (10 patients). Protein and salt intakes constituted uncontrolled variables in these studies, but other experiments indicated that their influence on serum lipids was not important.

During the period of normal fat and cholesterol intake the serum lipid levels of the patients did not differ significantly from those of healthy subjects of the same age. On a fat intake between 50 and $60 \mathrm{gm}$. per day the levels were not significantly altered. With restriction of dietary fat to below $40 \mathrm{gm}$. per day, changes in the serum lipids were observed which varied greatly among individuals. Although total cholesterol level was decreased significantly in $68 \%$ of the patients, there were significant increases in $12 \%$ of the series. Eighteen per cent of the patients developed a lipemia characterized by turbid serum, slight elevation of free cholesterol and substantial elevations of lipid phosphorus and neutral fat levels.

Ultracentrifugal serum lipoprotein studies performed in twelve of these patients by Dr. Gofman revealed that the rice diet reduced the $S_{\boldsymbol{r}} 12-20$ complex level in only one patient; substantial rises occurred in five patients.

It is concluded that drastic dietary fat restriction in patients with hypertension evokes changes in the serum lipids which are highly variable among individuals. Whereas two-thirds of patients exhibit decreases in total serum cholesterol, nearly one-fifth of patients develop lipemia and nearly one-half show increases in $S_{t}$ 12-20 lipoproteins.

Vitamin $B_{12}$ Levels in Pernicious Anemia. JoHN W. HARRIs, Boston, Mass. (Introduced by William B. Castle).

Employing Euglena gracilis var. bacillaris for Vitamin $B_{12}$ assay, it was consistently possible to detect concentrations of $6 \mu \mu \mathrm{g} / \mathrm{ml}$ and to recover $70-90 \%$ of crystalline material added to normal or pernicious anemia sera. "Free- $B_{12}$ " was assayed directly on sterile samples; "Total- $\mathrm{B}_{12}$ " was assayed on samples previously heated at $100^{\circ} \mathrm{C}$ for one hour-this procedure having been found to make available to the organism the maximum $B_{13}$ activity.

Ten normal sera averaged $23 \mu \mu \mathrm{g}$ free- $\mathrm{B}_{12} / \mathrm{ml}$ and $55 \mu \mu \mathrm{g}$ total- $\mathrm{B}_{22} / \mathrm{ml}$; normal erythrocytes, $36 \mu \mu \mathrm{g}$ total$\mathrm{B}_{\mathrm{1}_{2}} / \mathrm{gm}$; and normal peripheral blood or bone marrow buffy coats, $831 \mu \mu \mathrm{g}$ total- $\mathrm{B}_{12} / \mathrm{gm}$.

Sixteen sera from pernicious anemia patients in relapse contained no detectable free- $B_{12}$ and averaged $1 \mu \mu \mathrm{g}$ total-
$\mathrm{B}_{12} / \mathrm{ml}$; erythrocytes showed no detectable $\mathrm{B}_{12}$ activity; but peripheral blood or bone marrow buffy coats averaged $121 \mu \mu \mathrm{g}$ total- $\mathrm{B}_{12} / \mathrm{gm}$.

Sera from three patients with megaloblastic anemia but with free $\mathrm{HCl}$ in gastric juice contained 82, 60, and 32 $\mu \mu \mathrm{g}$ total- $\mathrm{B}_{12} / \mathrm{ml}$.

Sera from five groups of pernicious anemia patients maintained on different amounts of crystalline $B_{12}$ or liver extract given intramuscularly every 4 weeks were studied. The sera were obtained 4 weeks after the last injection of a series lasting eighteen months. The average total- $\mathrm{B}_{12} /$ $\mathrm{ml}$ of individual patients from each group was as follows: Group I, followed as relapse rate controls on saline injections alone, $6 \mu \mu \mathrm{g} \mathrm{B} \mathrm{B}_{12} / \mathrm{ml}$; Group II on $5 \mu \mathrm{g} \mathrm{B} \mathrm{B}_{12}, 18 \mu \mu \mathrm{g} /$ $\mathrm{ml}$; Groug III on liver extract assayed to contain $5 \mu \mathrm{g} \mathrm{B}$ $25 \mu \mu \mathrm{g} / \mathrm{ml}$; Group IV on $15 \mu \mathrm{g} B_{22}, 31 \mu \mu \mathrm{g} / \mathrm{ml}$; and Group $\mathrm{V}$ on liver extract assayed to contain $15 \mu \mathrm{g} \mathrm{B}_{12}$, $32 \mu \mu \mathrm{g} / \mathrm{ml}$.

\section{The Effect of Hemorrhage and the Pyrogenic Reaction on Hepatic Circulation in Dogs. Henry Heineman, Cheves McC. Smythe and Paul A. Marks, New York, N. Y. (Introduction by Stanley E. Bradley).}

The effect of rapid hemorrhage on hepatic circulation and hepatic cellular function was studied in 12 dogs anesthetized with nembutal. The hepatic blood flow (EHBF) was estimated as the bronsulfalein (BSP) clearance and BSP extraction was determined directly using the technique of hepatic venous catheterization. The arterial pressure was measured directly with a strain gauge and recording galvanometer.

The average control EHBF was $31 \mathrm{ml} / \mathrm{kg}$ body weight/ min. Immediately following rapid phlebotomy of 1.3 to $3.9 \%$ of body weight, EHBF fell to 28 to $80 \%$ of control levels in 10 of 12 dogs. There was a concomitant drop in mean arterial pressure of $20-59 \mathrm{~mm} \mathrm{Hg}$. During this period of decrease in EHBF there was an increase in hepatic vascular resistance. Associated with these changes there was an increase in arterial-hepatic vein oxygen difference and an increase in BSP extraction by the liver. These data suggest that hepatic vasoconstriction occurred immediately following hemorrhage.

During spontaneous recovery, EHBF rose to 71 to 99\% of control values within 20 to 50 minutes following blood loss. This "recovery" of EHBF occurred although the arterial blood pressure consistently remained lower than control levels and did not tend to rise.

In two animals there was no decrease in EHBF following hemorrhage. Both had pyrogenic reactions prior to phlebotomy and EHBF increased significantly during this reaction and persisted elevated through the posthemorrhagic period.

The effect of pyrogen (typhoid vaccine) on EHBF was studied further in four dogs. Within 10 to 20 minutes after pyrogen was administered EHBF increased 32 to $119 \%$ above control values, with a decrease in BSP extraction by the liver. 
Dynamic Aspects of Carbon Dioxide and Urea Metabolism in the Human Studied with Radioactive Carbon. Leon Hellman and Maxwell L. Eidinofr, New York, N. Y. (Introduced by Konrad Dobriner).

Radioactive carbon $\left(\mathrm{C}^{14}\right)$ labelled precursors have been used to study the quantitative aspects of the formation of urea from carbon dioxide in the human. The rates of synthesis and the total amounts (pools) of carbon dioxide and urea present in the body have also been determined.

Bicarbonate labelled with $\mathrm{C}^{\mathbf{1 4}}$ was administered intravenously and the expired labelled $\mathrm{CO}_{2}$ was collected at short time intervals. Simultaneous urine collections were made and the specific activity of the $\mathrm{C}^{14}$ labelled urea, which could only have been derived from the administered $\mathrm{C}^{14}$ bicarbonate, was determined. Over $80 \%$ of the administered bicarbonate was eliminated as $\mathrm{CO}_{2}$ within 8 hours. The $\mathrm{CO}_{2}$ specific activity curve reveals an initial rapid mixing of the labelled bicarbonate with the body fluids, followed by a decrease in activity which reflects the rate of production and the size of the carbon dioxide pool of the body. In a $70 \mathrm{~kg}$ man, the $\mathrm{CO}_{2}$ pool renews itself or turns over approximately once each hour and consists of 30 Grams of carbon dioxide, chiefly in the form of bicarbonate.

The mathematical analysis of the curves for urea and carbon dioxide is in accord with the concept that the carbon of urea is directly derived from the carbon dioxide pool. The urea pool consists of approximately 12 Grams of urea distributed in a volume equal to the total body water and turns over once every 12 hours. The size and turnover rate of the urea pool calculated above has been confirmed in an independent manner by administering labelled urea and measuring the extent to which it is diluted by the urea present in the body.

This relationship between $\mathrm{CO}_{2}$ and urea was shown to be a general one by comparing excreted $\mathrm{CO}_{2}$ and urea activities after the administration of $\mathrm{C}^{\mathrm{14}}$ acetate and methionine which form carbon dioxide in the course of their metabolism.

Rapid Changes in Plasma P.B.I. Levels in Euthyroid and Hyperthyroid Subjects. BAsIl S. Hetzed, DoRothy S. dE LA Haba and Lawrence E. HinkLe, JR., New York, N. Y. (Introduced by Harold G. Wolff).

The plasma protein bound iodine (P.B.I.) has been shown to be a measure of the blood thyroid hormone and is being used for the diagnosis of thyroid disease. In this investigation hitherto unsuspected fluctuations have been found in association with exposure to stressful life experiences.

The alkaline ash method of Barker has been used and found to give satisfactory results so that deviations of greater than $\pm 12 \%$ have been significant. Serial observations at hourly intervals have been made over 3 to 7 hour periods in euthyroid and hyperthyroid patients who had not previously ingested iodine in any significant amounts. Under neutral conditions the P.B.I. has been observed to be remarkably constant during the period of observation in both euthyroid and hyperthyroid subjects.
However, during experiences of stressful significance to the individual marked fluctuations have been found-rises of as much as $100 \%$ one to three hours afterwards in some subjects, or falls of up to $60 \%$ in others.

The pertinence of these findings to Graves' disease is currently under study. However, it is concluded that the possibility of these changes should be taken into account in using the P.B.I. as an aid in clinical diagnosis.

Normal and Impaired Retinal Vascular Reactivity to Oxygen and its Correlation with Cerebral Vascular Reactivity to Carbon Dioxide. John B. Hickam,* James F. Schieve and H. O. Sieker, Durham, North Carolina.

It is known that normal retinal arteries and veins will constrict when the arterial blood oxygen tension is increased and dilate when it is lowered. The present study was undertaken to determine whether this reaction is altered in persons with known vascular disease, and whether retinal vascular reactivity (RVR) to this stimulus can be correlated with some measurement of cerebral vascular reactivity (CVR).

Eyeground photographs were made while the subjects breathed first air and then tank oxygen. The diameter of the larger retinal vessels was measured from the photographs, using a low-power microscope with a scale. RVR was expressed as per cent shrinkage in vessel diameter while breathing oxygen. CVR was measured as increase in cerebral blood flow (cc/ $100 \mathrm{grams} \mathrm{brain} / \mathrm{min}$.) resulting from inhalation of $5 \%$ carbon dioxide.

Studies of RVR were made on 24 normal persons, 21 hypertensives, and 14 diabetics. In addition, RVR and CVR were measured together in 17 persons. Normal persons (ages 16 to 83) showed a slight decline in RVR with advancing age. The arteries of the hypertensive and diabetic patients showed significantly less reactivity $(P<.01)$ than normal. The veins of the diabetics also showed less reactivity $(P<.05)$ than normal. There was a significant correlation $(r=.793)$ between reactivity of the retinal arteries to oxygen and increase in cerebral blood flow on $5 \%$ carbon dioxide. There was no correlation between reactivity of the retinal veins and CVR.

It is concluded that the retinal arteries usually undergo significant loss of their reactivity to oxygen in patients with hypertension and diabetes, and that this loss is well correlated with loss of the ability to increase cerebral blood flow in response to carbon dioxide. The loss of RVR in patients with disorders affecting the vessels, and the simplicity of the technique, suggest that the subject deserves further study.

Isolation and Concentration of Human Blood Platelets: Their Properties in vitro and in vivo. ERWIN $O$. Hirsch, Frank H. Gardner and E. Donnall Thomas, Boston, Massachusetts. (Introduced by Samuel $L$. Gargill).

Whole blood was centrifuged at $4^{\circ} \mathrm{C}$ in plastic containers at $400 \times \mathrm{G}$ for 15 minutes. Supernatant plasma 
containing 50 to $90 \%$ of the platelets and as little as $2 \%$ of the white cells was siphoned into plastic containers, spun at $1,000 \times G$ for 10 minutes, and the platelets then washed twice with resuspension between washings. Preparations containing 1,000,000 platelets per cu. mm., 600 white cells and practically no red cells can be routinely obtained in this fashion.

When $.066 \mathrm{M}$ sodium citrate (one part to nine parts of blood) was used as anticoagulant and one-hour old platelet-rich plasma was infused into a patient with aplastic anemia, platelets survived normally. After being washed twice with $.066 \mathrm{M}$ sodium citrate platelets did not appear to be viable in vivo. In vitro they produce clot retraction and normal clotting time in thrombocytopenic plasma. Serum prothrombin time, however, is lengthened far beyond that of fresh plasma containing the same number of platelets.

When $1 \%$ disodium ethylene-diamine-tetraacetate was used as an anticoagulant and platelets were washed twice with saline, serum prothrombin time was the same as that of fresh plasma containing the same number of platelets.

Washed platelets exhibit oxygen consumption and utilize glucose. They produce $\mathrm{CO}$, and lactic acid. Platelets from a patient with scurvy exhibited normal oxygen consumption. On storage, platelets lose their ability to cause clot retraction within 24 hours. Their effect on clotting time and serum prothrombin time persists for weeks. Oxygen consumption decreased rapidly and was always zero after three days' storage.

Spermine and Tubercle Bacilli. JAMrs G. HIRSCH, New York, New York. (Introduced by Gardner Middlebrook).

In previous studies a substance inhibiting the growth of mammalian tubercle bacilli in vitro was isolated from tissues in crystalline form. When added to a modified Dubos medium, a final concentration of 0.001 per cent ( $0.01 \mathrm{mg}$. per cc.) of this crystalline material completely inhibited the growth of several strains of human and bovine mycobacteria, regardless of the size of the bacillary inoculum. Other microorganisms, including avian tubercle bacilli, saprophytic mycobacteria and various non-acid-fast bacteria, were much less susceptible or completely resistant to the inhibitory action of this substance.

After a variety of chemical studies, the crystalline material was identified as the phosphate salt of an organic base, spermine. This polyamine $\left(\mathrm{NH}_{5}-\left(\mathrm{CH}_{2}\right)_{-}-\mathrm{NH}-\right.$ $\left.\left(\mathrm{CH}_{2}\right)_{2}-\mathrm{NH}-\left(\mathrm{CH}_{2}\right)_{-}-\mathrm{NH}_{2}\right)$ has been demonstrated to occur widely in animal tissues, including those of man. Little is known concerning the origin, function and fate of the spermine present in the body.

Recent investigations inquiring into the mechanism of action of spermine on tubercle bacilli reveal that suppression of growth occurs only if an unidentified component of certain sera, serum fractions and aqueous extracts of some organs is present in the system. This activating substance is a protein with the characteristics on an $\alpha$ globulin. Very small quantities of this protein, much less than 1 microgram per cc., bring about the full inhibitory action. Further studies of the nature of this activator and of the mechanism by which it produces, in conjunction with spermine, a toxic effect on tubercle bacilli are in progress.

Effects of Head-up Tilting, Pitressin, ACTH, Cortisone and Desoxycorticosterone Glucoside on Excretion of Sodium and Chloride. Bernard C. Holland, Durham, North Carolina. (Introduced by Eugene A. Stead, Jr.).

Motionless standing causes retention of water, sodium and chloride. Previous studies have shown that the retention of water is related to activity of pituitary ADH mechanism, but the factors responsible for the salt retention remain unknown. The effect of head-up tilting at angle of $45^{\circ}$ was compared with the effect of administration of pitressin, ACTH, cortisone and desoxycorticosterone glucoside.

Normal subjects were placed on a diet containing 24 gm. of sodium chloride per day. After a 12-hour fast, they were given $1 \mathrm{gm}$. of $\mathrm{NaCl}$ in $200 \mathrm{cc}$. of water each hour for six hours. Subjects either remained horizontal without medication or at the end of the second hour were tilted or given medication.

Tilting uniformly caused a marked decrease in excretion of sodium, chloride and water without a significant fall in eosinophiles.

Pitressin in doses of 3 milli-units per kilogram caused no change in excretion of sodium and chloride, and no fall in eosinophiles.

Cortisone (200 mgm. orally) or ACTH (20 mgm. i.v.) had no consistent effect on excretion of sodium. Chloride excretion tended to rise. There was marked eosinopenia.

Desoxycorticosterone glucoside (50 mgm. i.v.) caused an increase in chloride excretion without a consistent effect on sodium excretion. The eosinophiles did not fall.

Neither pitressin, cortisone, ACTH nor desoxycorticosterone glucoside causes in the first four hours the pattern of sodium and chloride retention that is produced by head-up tilting. Tilting causes marked salt retention without a marked fall in eosinophiles.

Role of Tissue Binding in Local Erythroid Maturation Following Instillation of Vitamin $B_{18}$ into Marrow Cavity. Daniel L. Horrigan and Robert W. Heinle,* Cleveland, Ohio.

A local erythroid maturation effect following instillation of vitamin $B_{12}$ directly into marrow cavity of persons with pernicious anemia was described previously. The interpretation of this observation was that the vitamin was capable of inducing maturation without first being altered.

Other investigators have shown that vitamin $B_{12}$ exists in the blood in bound form, that many substances are capable of binding, and that the unbound vitamin is ineffective in causing erythroid maturation in vitro.

In this study, the observations indicate that the vitamin is bound at the site of instillation but that binding capacity 
is limited. After instillation of $1 \mathrm{mcg}$. into the marrow cavity of three patients, previously reported, and of 0.25 mcg. in another, maturation occurred only at the site of injection without other hematologic response. In two patients, however, instillation of $1 \mathrm{mcg}$. was followed by a systemic response, and generalized marrow maturation, although the latter was more advanced at the site of instillation.

These findings suggest that in the patients who showed local maturation only, the vitamin was completely bound at the injection site, whereas in those having a systemic response, only part was bound locally, the remainder being available for systemic utilization. Local binding capacity is thought to be related to the degree of tissue vitamin deficiency and varies in different patients. That the effective binding substance and gastric intrinsic factor are the same has been suggested but not proven. If combination with a binding substance is necessary before vitamin $B_{12}$ becomes active, a deficiency of tissue binding substance might be the basic defect in certain refractory macrocytic anemias associated with megaloblastic or abnormal normoblastic bone marrow states as have been described in cirrhosis and certain other conditions.

\section{Viremia in Poliomyelitis Following Infection by a Nat- ural Route. Dorothy M. Horstmann,* New Haven, Conn.}

For the past 40 years viremia in human poliomyelitis has been considered to be of rare occurrence, and unimportant in the pathogenesis of the disease. These conclusions have been based on the largely negative results of attempts to demonstrate the virus in the blood of patients. In fact, only one positive result has been reported, and this with blood drawn from an abortive case within several hours of onset. However, the negative results may have been due to the search for virus having been made too late in the course of the human disease, and for this reason the problem was attacked experimentally in cynomolgus monkeys ( $M$. Cynomolgus) and chimpanzees ( $P$ an satyrus) following infection by a natural route, i.e. orally. In 4 separate experiments, animals which had been fed Y-SK or Egypt strains of poliomyelitis virus (Lansing and Brunhilde types, respectively) were bled from the femoral vessels daily or every other day for 5-7 days following virus feeding. Blood specimens from the chimpanzees and from the monkeys which subsequently became paralysed, were then tested for virus by intracerebral inoculation into rhesus monkeys. Three of 4 chimpanzees and 9 of 12 monkeys to whom virus had been fed in this manner were thus demonstrated to have poliomyelitis virus in the blood between the 4th and 6th days after virus ingestion; in some animals on all 3 consecutive days. The chimpanzees remained asymptomatic, but in the case of the monkeys, viremia preceded the onset of paralysis by 3 to 7 days.

These results in experimental animals reopen the possibility that viremia may occur in human poliomyelitis. It seems likely that it may occur during the incubation period of the human infection, or in association with the minor illness or "first phase" of the disease.
A Model of Plasma Iron Kinetics in Human Subjects. Rex L. Huff,* Paul Elmlinger, Robert Mortimer, Hal Anger and C. A. Tobias, Berkeley, California.

On the basis of total plasma iron, body surface counting of iron 59, and plasma iron 59 data, a kinetic model has been hypothesized. The data are from forty patients with hematopoietic disease and from seven normal subjects. They were given single intravenous injections of iron 59 attached to globulin-IV-7 (Cohn). The model provides the basis for rate computation for the movement of plasma iron. The plasma data of the normal subjects were analyzed into a polynomial of three decaying exponentials having half times of approximately $0.1,0.5$, and 3 days. The initial component accounted for dilution in a quantity of iron approximately forty times that of the plasma iron. This represents iron in equilibrium with that of the plasma and possibly is some fraction of storage iron as well as that in pigment forming tissueschiefly those tissues forming hemoglobin. The subsequent dilution of tracer in this equilibrium pool is thought to be related to the incorporation of the iron into pigment. The appearance of a third slow component may represent the turnover of storage iron.

The data of the patients differed in several ways, some of which required a more complicated model than that of the normal subjects. The graphic analysis of some patients' data resulted in polynomials of more than three exponentials. Half times of the first component of a magnitude compatible with complete portal clearance of the tracer correlated with in vivo data showing primarily hepatosplenic turnover, similarly half times of the first component compatible with complete bone marrow clearance of tracer correlated with in vivo data indicating marrow accumulation. Liver storage of iron 59 and depression of erythropoiesis was uniformly associated with a long half-time of the first component as well as a decreased amplitude.

Homologous Transplantation of Human Kidneys. DAvid M. Hume, John P. Merrill and Benjamin F. Miller,* Boston, Massachusetts.

Homologous transplantations of human kidneys have been studied in six patients. The recipients were patients in far-advanced, irreversible, chronic uremia. Donor kidneys were obtained during surgical procedures in three instances, and from cadavers in the remainder.

The transplanted kidneys were placed in the recipient's thigh, with anastomosis of renal artery to the profunda branch of the femoral artery and renal vein to the common femoral vein. The ureter was brought out through the skin, permitting collection of urine directly from the transplanted kidney.

The periods of ischemia of the donor kidneys lasted from 14 minutes to 205 minutes. The transplanted kidney which had been ischemic for the longest period functioned better than the others, but no conclusion can be drawn from such a small series of observations. The phenolsulphonthalein test on this kidney indicated $22 \%$ excretion in 2 hours as against $0 \%$ in the bladder urine. On 
the 21st post-operative day, clearances on the transplant were obtained: inulin, $14 \mathrm{cc} / \mathrm{min} / 1.73 \mathrm{M}^{2}$; urea, $6.4 \mathrm{cc} /$ min.; creatinine, $19 \mathrm{cc} / \mathrm{min}$; renal plasma flow, 112 $\mathrm{cc} / \mathrm{min}$. During the several weeks that this transplant functioned, the blood urea nitrogen was reduced from 130 to $40 \mathrm{mgms} \%$.

Three transplanted kidneys never developed measurable function because of technical difficulties. The other three kidneys went through a period resembling acute tubular necrosis, with anuria for 8 to 14 days, following which they produced urine, attaining in one instance a volume of $2800 \mathrm{ml}$ daily. Two transplants functioned considerably longer than any homotransplants reported in animals.

Infection was the chief difficulty encountered. It eventually destroyed all the transplants. It is possible that concomitant administration of cortisone and ACTH may have intensified these infections.

\section{A Dextrorotatory Urobilin from Feces Following Anti-} biotic Therapy. G. Watson James, III, ANne ANDERSON and VIVIAN WILKERSON, Richmond, Virginia. (Introduced by Bernard M. Jacobson).

Stercobilin, strongly levorotatory, has been isolated from feces by Watson, and urobilin IX, $a$, optically inactive, has been prepared synthetically from mesobilirubinogen, but is also present in small quantities in some pathological urines. Both of these compounds have been thoroughly described. A dextrorotatory urobilin in infected bile, and in feces of a patient following aureomycin has been reported, but not isolated in quantities for adequate study.

From the feces of patients with various hemolytic anemias, stercobilin was isolated. Then aureomycin or terramycin was given for several days and urobilinogen formation depressed. When the Ehrlich reaction had returned the feces were subjected to the same isolation procedure, and now a crystalline dextrorotatory urobilin, different from stercobilin was regularly obtained in large quantities. It was still present in the feces for as long as sixty days after the antibiotics were discontinued. In one patient with $\mathrm{N}^{16}$ labelled red cells the heavy isotope content of the stercobilin and $d$-urobilin were the same suggesting a common source. During chloromycetin administration only stercobilin was obtained.

$d$-Urobilin possesses some of the chemical and physical properties of both urobilin IX, $a$, and stercobilin. Two crystal forms have been observed. The chemical reactions are more similar to those of urobilin IX, $a$, and oxidation to ferrobilin and glaucobilin is easily performed. The Ehrlich reaction of $d$-urobilinogen is both quantitatively and qualitatively the same as stercobilinogen. Other properties, a possible structural formula, and its significance will be discussed.

The Effect of a Single Injection of ACTH upon Urinary Excretion of Sodium, Potassium and 17-Ketosteroids. William McK. Jefferies, Alfred K. Bochner and Ralph I. Dorfman, Cleveland, Ohio. (Introduced by Joseph M. Hayman).
Adrenal cortical activity is manifested by effects which can be divided into three main categories: (1) regulation of electrolyte metabolism, characterized by a retention of sodium and a loss of potassium (DOCA effect), (2) regulation of carbohydrate metabolism (S hormone effect), and (3) an androgenic or protein anabolic effect ( $\mathrm{N}$ hormone effect). The current test of adrenal cortical response to $25 \mathrm{mgm}$. ACTH i.m. involves changes in circulating eosinophiles and urinary excretion of uric acid, both reflections of changes in S hormone effect, only one of the 3 phases of action.

The effect of administration of $25 \mathrm{mgm}$. ACTH i.m. upon urinary excretion of sodium, potassium and 17ketosteroids in specimens collected according to the same schedule as the Thorn test was studied in 10 normal young men. In every instance an increase in excretion of $\mathrm{K}$ and 17-ketosteroids occurred, whereas a decline in excretion of these substances was seen in three patients with Addison's Disease tested in the same fashion. In 8 tests urinary $\mathrm{Na}$ excretion decreased, but decreases were also seen in the Addisonians. Control studies indicated that these effects could not be attributed to changes in urine volume or to diurnal variation. Following a single alarming stimulus, the change in excretion of these substances was found to correspond well to that following a single injection of ACTH. A case of adrenogenital syndrome showed a lack of increase in excretion of $\mathrm{K}$ with a good increase in excretion of 17-ketosteroids following a single injection of ACTH.

Hence, by modifying the Thorn test to include determinations of changes in urinary excretion of $\mathrm{Na}, \mathrm{K}$ and 17-ketosteroids, a more complete reflection of the pattern of response of the adrenal cortex to a single stimulus may be determined.

Hepatitis in Mice of Presumed Viral Origin. JANET Jordan, Charles I. Leftwich and George S. Mirick,* Baltimore, Maryland.

The saline extract of the liver of a patient (CB) who died of hepatitis twenty-seven days after exposure, was injected intraperitoneally into mice given $0.1 \%$ urethane to drink. Monthly serial intraperitoneal transfers of organ extracts were made in three week old urethanized mice. In the fourth passage 2 mice developed a disease characterized by ascites, hepatomegaly and splenomegaly. Microscopic sections of the liver showed central and periportal mononuclear cell infiltration and occasionally central or diffuse necrosis. Eleven subsequent positive monthly serial transfers have been completed. Organ extracts and ascitic fluid diluted to $1 / 1000$ and blood were infectious when inoculated parenterally but experimental, oral and intranasal inoculations were negative. The incubation period is 3-4 weeks. Most mice survive with anatomical restoration of the liver and the appearance of some immunity. Many control series of mice were negative prior to the isolation of the agent in the laboratory. Since that time the disease has occasionally been seen in uninoculated control mice as well as control passage series inoculated with human material thought to 
be normal. Rabbits, guinea pigs, hamsters, monkeys and embryonated eggs inoculated with material infectious for mice remained well. Bacteriological cultures have been negative and the available evidence indicates that the disease is not due to a virus of the psittacosis group or to LCM, PVM, the mouse hepatitis virus of Gledhill and Andrewes, the agent of Ehrlich's ascitic tumor, leptospira, or to ectromelia. Streptomycin, terramycin, chloramphenical and sulfadiazine do not inhibit the disease. All attempts to neutralize the agent with convalescent serum from patients with hepatitis, $\gamma$ globulin and convalescent mouse serum have failed. The agent has resulted in gastro-intestinal symptoms and abnormal liver function tests when inoculated into volunteers.

Transmission of Acute Non-Bacterial Gastroenteritis to Volunteers: Evidence for Two Different Agents. WILLiAM S. JoRdan, JR.,* Irving Gordon, Richard G. Hodges and William R. Dorrance, Cleveland, Ohio.

Clinical and epidemiological data suggested that at least two types of infectious gastroenteritis, afebrile and febrile, occur in families. Studies comparing the febrile type with a known strain (Marcy) are reported here. Bacteria-free supernate of stool from a febrile case (FS-I7549) produced anorexia, abdominal cramps and loose stools in four of eight volunteers after 26 to 30 hours. Three of the four experienced vomiting and fever.

The illnesses induced by the febrile inoculum differed from those induced by the Marcy strain, a filterable agent obtained from an institutional outbreak. When fed as stool supernates, this agent has produced afebrile illnesses characterized chiefly by watery diarrhea.

Supernates from sixth human passage Marcy and first passage FS stools were fed to volunteers in individual isolation. Six of seven men given $7 \mathrm{ml}$ Marcy became ill within 24 to 120 hours. One of eight given $2 \mathrm{ml} \mathrm{FS}$ developed a temperature of 101.8, nausea, anorexia and headache in 20 hours; five noted mild abdominal symptoms after 20 to 30 hours. Sixteen days later, the men ingested the same inocula, but the amount of FS was increased to $10 \mathrm{ml}$. No illnesses occurred in the Marcy group. In the FS group, one definite illness occurred at 30 hours; two other men had mild symptoms.

Thirteen days later, cross immunity was tested. Marcy inoculum induced diarrheal disease in seven of eight men, including two previously sick with FS. FS inoculum induced febrile disease in three of seven men, all previously sick with Marcy, within 21 to 30 hours.

Lack of heterologous immunity and the different clinical pictures produced suggest that one of the types of gastroenteritis observed in families, the febrile type (FS), differs from the Marcy type and presumably represents a new entity.

Further Studies on the Antidiuresis and Decrease in Sodium Excretion During Venous Congestion of the Limbs: Its Prevention in Normal Subjects by a Large Transfusion; Its Absence or Presence in Cardiac Patients with or without Congestive Failure. WALTER E.
Judson, J. Donald Hatcher, Meyer H. Halperin, and RoBert W. Wilrins,* Boston, Massachusetts.

In previous reports to the Society it was shown that: 1. Antidiuresis and decreased sodium excretion, and usually decreased renal blood flow, occur during venous congestion of the limbs in normal subjects and in hypertensive patients before and after splanchnicectomy.

2. Decreased sodium excretion without antidiuresis may be produced by venous congestion of the limbs in normal subjects under osmotic (mannitol) diuresis, or in patients with diabetes insipidus.

In the present work it was found that:

1. Antidiuresis and decreased sodium excretion do not occur during venous congestion of the limbs of normal subjects who concomitantly are given a large (1 to 1.5 L.) blood transfusion, but may occur if a smaller volume of blood is given. This suggests that a decrease in the effective circulating blood volume, and not peripheral factors such as venous distention, edema formation or discomfort in the limbs, is the important stimulus to the antidiuretic response.

2. Antidiuresis and decreased sodium excretion may occur during venous congestion of the limbs in certain patients with severe heart disease.

3. Antidiuresis and decreased sodium excretion do not occur during venous congestion of the limbs in certain other patients with severe heart disease and congestive failure.

4. Measurements of cardiac output (Fick) and of pulmonary arterial, right ventricular, and peripheral arterial pressures during venous congestion of the limbs in selected normal and cardiac subjects suggest that antidiuresis occurs when there is a decrease in cardiac output or peripheral arterial pressure, but does not occur when there is no decrease in cardiac output or peripheral arterial pressure, even though there may be decreases in pulmonary arterial and right ventricular pressures.

Ascorbic Acid Requirements in "Damage" and Its Relationship to Adrenocortical Activity. ROBERT M. KARK,* Robert E. Chapman and C. Frank Consolazio, Chicago, Illinois.

Levenson et al. (Ann. Surg. 124: 840, 1946) have shown precipitous falls in urinary ascorbic acid in "damaged" surgical patients during the "catabolic phase" of stress, despite parenteral therapy with large (normally saturating) amounts of ascorbic acid. These data are accepted widely as evidence that requirements for ascorbic acid are increased after surgery and other insults. The demonstration, in rats, that adrenocortical activity consumed adrenal ascorbic acid during and after "stress" offered a seemingly rational explanation for the so-called increased utilization of ascorbic acid.

This study reports effects of therapy with adrenocorticotropin or cortisone on: the plasma, whole blood, fecal and urinary ascorbic acid; eosinophils; and urinary 17. ketosteroid excretion, in patients and healthy individuals, who lived on fixed dietary regimens and fixed (analyzed) ascorbic acid intakes, in a metabolic unit. Available data 
showed that: (a) In classical scurvy, good adrenocortical response to A.C.T.H. was indicated by drop in eosinophils and rise in 17-ketosteroid excretion. Ascorbic levels in blood, urine and feces did not change. (b) In individuals "saturated" with ascorbic acid, urinary ascorbic acid increased considerably during A.C.T.H. or cortisone therapy and during adrenal rebound. Blood, urine and fecal ascorbic acid levels did not change. (c) In "unsaturated" individuals change in urinary ascorbic acid was variable but retention of urinary ascorbic acid did not occur.

The data indicate that in man: consumption of ascorbic acid by the adrenal for synthesis of cortical hormone cannot be considerable; adrenocortical activity cannot account for urinary retention of ascorbic acid seen after surgery; diuresis of ascorbic acid occurs with adrenocortical activity when large amounts of ascorbic acid are ingested; perhaps comparable with adrenocortical glycosuria.

On the basis of the above and studies in patients given A.C.T.H. after surgery, speculations will be made on ascorbic acid metabolism in the catabolic phase of "damage."

The Effect of Various Bacterial Derivatives and of Physical Injury on the Lysozyme-like Activity of Human Leukocytes. GRACE P. KERBY, Durham, North Carolina. (Introduced by D. T. Smith).

Using an in vitro method of assay of a lysozyme-like enzyme present in human leukocytes, an increased enzymatic activity has been demonstrated on exposure of the leukocytes to various bacterial derivatives (Proteus vulgaris, Ps. aeruginosa, E. coli, B. subtilis and Friedlander B carbohydrates; meningococcal filtrate; pneumococcal type III SSS). In some instances (Proteus vulgaris, Ps. aeruginosa, E. coli and B. subtilis carbohydrates) the per cent rise in activity over the control has tended to be measurably proportional to the amount of substance added. In other instances, the method has not differentiated amounts of the substance added, within the range tested. The enzymatic activity of leukocytes as measured varies from day to day in the same individual and from individual to individual, but increased activity in the presence of the test bacterial derivative as compared to the control has been consistent.

The enzymatic activity is increased more markedly by lysis of the leukocytes by saponin or distilled water than by exposure to the bacterial derivatives. Also, progressive decrease in the concentration of a suspending saline solution below isotonicity produces progressive increase in enzymatic activity of the suspended leukocytes. The possibility is considered, therefore, that the increased activity noted in the presence of the bacterial derivatives reflects damage to the leukocyte wall, with release of enzymes into the suspending medium.

The findings to date suggest that the method may be adaptable to a gross, in vitro titration of various substances such as the bacterial pyrogens which are damaging to leukocytes. Studies are in progress also to deter- mine the effect of the bacterial derivatives on the leukocytes of individuals with bacterial hypersensitivities and with induced pyrogen tolerance. The usefulness of the method as a means of determining the metabolic intactness of stored leukocytes remains to be clarified; preliminary studies suggest this possibility.

Paradoxical Effects of Cortisone on Influenza B Virus Infection of the Chick Embryo. Edwin D. KILBourne, New Orleans, Louisiana. (Introduced by Thomas M. Rivers).

Investigations pursuant to previously described observations of increased virus concentrations in fertile hen's eggs injected with cortisone have revealed in eggs so injected a diminution or delay in the toxic effects of Influenza B virus multiplication concomitant with the attainment of allantoic fluid virus concentrations equal to or far exceeding those of controls. Decreased toxicity was manifested by increased survival time and lower allantoic fluid protein concentration of eggs administered cortisone.

The paradoxical effects of increased survival time and increased virus concentration observed with the administration of the same dose of cortisone have been partially clarified by a study of time-dosage relationships. The intra-allantoic injection of chick embryos with $1 \mathrm{mg}$. of cortisone 1-20 hours after their inoculation with Influenza $B$ virus (Lee strain) results in no significant modification of survival time and in greatly increased virus concentrations. Injection of the same amount of cortisone $24-42$ hours subsequent to the initiation of infection induces a significant increase in survival time in the presence of concentrations of virus equal to or slightly exceeding those of controls. In contrast, injection of cortisone 18-24 hours prior to viral inoculation has resulted in the earlier death of cortisone-treated embryos.

Studies of Antibiotic Antagonism. William M. M. KIRBY* and JAMES J. AhERN, Seattle, Washington.

Jawetz has shown that chloramphenicol interferes with penicillin both in vitro and in vivo. In the animal experiments, effective antibiotic blood levels were present for only a few hours, and the results reflected the interference of chloramphenicol with the early bactericidal action of penicillin. The slower, but progressive, killing action of the combination, which often leads to sterilization of cultures in vitro, played little if any role. It seemed desirable, therefore, to repeat the experiments under conditions resembling those used in clinical therapy.

Mice were infected with group A streptococci, and chloramphenicol and a long-acting form of penicillin were injected repeatedly over a 3 day period. Under these circumstances, interference was not demonstrable. These results were interpreted as being due to the continued bactericidal action of the combination and, in addition, to the fact that time was allowed for effective phagocytosis to occur.

A 31 year old man with subacute bacterial endocarditis was treated with antibiotics for 3 years, relapsing when- 
ever therapy was stopped. The infecting organism, a viridans streptococcus, was killed in vitro by a combination of penicillin and chloramphenicol, and the patient was cured when these two antibiotics were administered for 25 days.

Twenty-five patients with pneumococcal pneumonia were treated with penicillin and 25 with penicillin and aureomycin, and the clinical course of the 2 groups was compared. No evidence of interference was observed.

Interference would appear to be of clinical importance only when recovery depends upon the early killing action of penicillin. Lepper and Dowling observed interference of aureomycin with penicillin in pneumococcal meningitis. Rapid killing is probably important in this instance because surface phagocytosis is relatively inefficient in the subarachnoid space.

It is concluded that interference of broad spectrum antibiotics with penicillin is seldom of clinical importance, and that under certain circumstances these combinations are desirable.

\section{Some Effects of Hexamethonium on Renal Circulation.}

Walter M. Kirkendall and James W. Culbertson, Iowa City, Iowa. (Introduced by Willis M. Fowler).

Eight patients had differential renal function studies before and after intravenous administration of hexamethonium chloride (C-6) in doses of 20 to $160 \mathrm{mgm}$. Two patients were feebleminded but otherwise normal, one had malignant hypertensive cardiovascular disease, two had coarctation of the aorta, and three had moderate essential arterial hypertension. Glomerular filtration rate (GFR) was measured by inulin and renal plasma flow (RPF) by p-aminohippuric acid plasma clearance. Usually three clearance periods were determined before and after administration of hexamethonium. All patients developed upright postural hypotension following injection, demonstrating effective autonomic ganglionic blockade.

In one normotensive person, the two patients with aortic coarctation, and the one with malignant hypertension renal plasma flow rates fell from 11 to 24 (av. 19) \% and glomerular filtration rates fell from 14 to 25 (av. 18) \% below control values. The filtration fraction (FF) was not significantly altered in any subject.

In the second normotensive subject and all three patients with essential hypertension RPF increased from 13 to 75 (av. 45) \%. There was little change in GFR, but FF decreased from 24 to 47 (av. 32) \% below control values.

Apparently the effect of hexamethonium on renal circulation differs in health and certain diseases. In one normotensive person without arteriolar spasm, in coarctation of the aorta (where the renal circulatory pattern may not be maintained by increased neurogenic tone), and in malignant hypertension with irreversible structural damage in the kidney, hexamethonium depressed RPF and GFR without changing FF significantly. In the other normotensive person, who had a "hypertensive" renal pattern before administration of hexamethonium, possibly from apprehension, and in three essential hyper- tensives changes in renal hemodynamics may have followed relaxation of spastic efferent arterioles indirectly from lowering of systemic arterial pressure or directly from release of neurogenic vascular tone in the kidney.

The Mechanism of Diuresis in Diabetic Acidosis. HARvey C. KNowles, JR., Cincinnati, Ohio. (Introduced by Eugene B. Ferris).

The major cause of dehydration in diabetic acidosis appears to be an osmotic polyuria which is accompanied by losses of electrolyte. The present study is concerned with verifying the flow-load relationship in this type of polyuria, and with the composition of the urinary solute load.

The studies concern 22 subjects of ages 20 to 50 years, in ketoacidosis, stuporous, with serum $\mathrm{CO}_{2}$ contents less than $13 \mathrm{meq} / 1$ and plasma urea nitrogen less than 30 mg.\%, free from shock and anuria. Urine specimens were collected before and just after the start of treatment. Blood specimens were collected in the pre-treatment period.

The urine flow could not be correlated with the plasma glucose level. There was some correlation between urine flow and glucose excretion. However, the relation between flow and total excreted solute load was found to be essentially linear and highly significant, similar to that observed in normal hydropenic subjects during osmotic diuresis. The mean total urinary osmolarity was found to be $543 \mathrm{M}$ Osmols/L and the mean urinary flow $3.54 \mathrm{cc} / \mathrm{min}$. Analysis of the mean osmolar composition of the urinary loads revealed glucose. to be the largest constituent, amounting to $42 \%$. Other constituents were ketones $7 \%$, sodium $6 \%$, potassium $6 \%$, phosphorus $4 \%$, and chloride $2 \%$. Of total nitrogen, urea nitrogen contributed $10 \%$, and the remaining nitrogen, if expressed entirely as ammonia, $19 \%$, of the osmolar composition.

Increased urinary flow was accompanied by increased sodium excretion but little change in potassium or phosphorus excretion.

Conclusions: (1) The diuresis in diabetic acidosis is osmotic in nature, with glucose the main urinary solute. (2) Sodium constitutes only a small part of the solute load; its excretion increases at higher rates of urinary flow.

Reduction of Tetrazolium by Growing Tubercle Bacilli. D. Koch-Weser, W. R. Barclay and R. H. Ebert,* Chicago, Illinois.

The reduction of colorless triphenyl tetrazolium to red formazan has been used as an indicator of the metabolic activity of tissues and bacteria. Previous investigations have been for the most part qualitative. This study reports quantitative metabolic studies of tubercle bacilli in Dubos' liquid medium. $2 \mathrm{mgm}$. of triphenyl tetrazolium were added to growing cultures of $\mathrm{H} 37 \mathrm{RV}$ and after 24 hours the amount of formazan present was estimated colorimetrically after extraction with ethyl acetate. The amount of tetrazolium reduced was directly proportional to the number of bacilli in the culture as determined 
turbidimetrically. An excess of asparagine added to the medium markedly increased tetrazolium reduction.

Streptomycin added to the cultures, in a concentration of $50 \mathrm{~g}$. per ml., 6 days after inoculation and at a time when the bacilli were in the logarithmic phase of their growth, immediately decreased the rate of growth and the amount of tetrazolium reduced. Complete inhibition occurred only 3 to 4 days after the antibiotic was added. Once complete inhibition of growth occurred the bacilli did not reduce tetrazolium as long as streptomycin was present in the medium.

Old cultures which had almost ceased growing reduced the same amount of tetrazolium per mgm. of bacilli as did young actively growing cultures.

The reduction of tetrazolium appears to be a useful reaction for studying the metabolism of tubercle bacilli.

\section{The Role of Desoxyribonuclease and a Nuclease Inhibitor from Leucocytes in the Lupus Erythematosus Cell Phe-} nomenon. N. B. KuRNICK, L. Schwartz, S. Pariser, S. Lee and W. Irvine, New Orleans, Louisiana. (Introduced by Albert Segaloff).

The observation of Klemperer et al, that depolymerization of the desoxyribonucleic acid (DNA) of mesenchymal cells is a constant feature in lupus erythematosus, suggests the action of desoxyribonuclease (DNase) in this phenomenon and Hargraves' "L.E. Cell Phenomenon."

The serum DNase activities of a number of normal individuals, patients with acute disseminated lupus erythematosus (L.E.) and patients with other diseases, indicated a wide range of activities for each group. L.E. sera tended to display elevated DNase activities, but there was no correlation between the level of DNase activity, the ability to elicit the L.E. phenomenon, and the severity of the disease. Furthermore, DNase activity, but not L.E. factor activity, is destroyed at $56^{\circ}$.

However, since cell nuclei contain DNase, its relation to the serum L.E. factor was investigated. We found that extracts of normal human WBC contained an inhibitor for serum DNase. The most potent of such inhibitors strongly inhibited the L.E. cell phenomenon, but this effect was not observed with less active inhibitor preparations. Since simple saline extraction removed some inhibitor from the WBC, washed WBC were tested as subtrates for the L.E. cell phenomenon, and found to be considerably more sensitive. Addition of an active inhibitor again suppressed the phenomenon.

Studies aimed at establishing the relationship of the inhibitors of DNase and of the L.E. phenomenon will be reported. The effect of enzymes, dialysis, incubation with normal and pathological sera, and with Fraction II, and salt fractionation will be presented. Attempts at localization of the inhibitors in cytoplasmic and chromosomal fractions of WBC and their species interactions, will be reported.

A hypothesis of the pathogenesis of the L.E. phenomenon and the general significance of the leucocyte DNase inhibitor will be discussed.
The Velocity of Wave Propagation in Muscular Arteries of Normotensive and Hypertensive Subjects. Milton LANDOWne,* Baltimore, Maryland.

A new method for the study of pulse wave velocity has provided information relating to the pressure-volume characteristics and elastic properties of the brachioradial artery in living subjects.

Repetitive pressure disturbances are introduced by small impacts delivered at one site along the vessel, being superimposed upon the ambient arterial pressure. These are detected and timed on arrival at another location by a suitable pressure transducer and indwelling needle. Several hundred waves may be recorded in a minute, permitting determination of wave velocity as a function of blood pressure.

A consistent relationship has been obtained in almost all subjects studied. Velocity (v) increases with pressure (p), and this variation usually is larger than the differences between subjects at any single pressure. With rare exceptions $\mathrm{dv} / \mathrm{dp}$ decreases as pressure increases. In many, but not all individuals, and for grouped information, the experimental data may be closely approximated (a.d $<2 \%$ ) for convenient representation as $\mathbf{v}$ (meters/ sec. $)=a^{b}(\mathrm{~mm} . \mathrm{Hg})$. Averaged data for 81 normotensive males aged 20-94 yield $a=2.4 ; b=0.45$, and for 14 hypertensive patients of various grades of severity $a=2.0 ; b=0.50$. The differences are not assigned significance. Marked variation in the degree of estimated medial sclerosis is also without striking effect.

The observations indicate that physiological distensibility of muscular arteries is relatively slight. With a change in arterial pressure from 50 to $150 \mathrm{~mm}$. $\mathrm{Hg}$, the computed maximal increase in vessel diameter is $2.5 \%$. Other evidence supports this value. The estimated elastic modulus of extension of the artery increases with pressure, consonant with the rubber-like properties of fibrillar proteins. Assuming vessel dimensions, a mean absolute value is of the order of $10^{\circ}$ dynes $/ \mathrm{cm}^{2}$, slightly lower than moduli reported for isolated muscle or elastic fibres.

Quantitative Antibody Studies in Man. Antibody Response in Leukemia and Allied Disorders. DANIEL L. LARson, New York City, N. Y. (Introduced by Joseph C. Turner).

Antibody responses following the administration of pneumococcus capsular polysaccharides I and II have been studied in patients with leukemia and allied disorders. Antibody determinations were carried out using the quantitative precipitin technique developed by Heidelberger. Results are expressed as micrograms of antibody nitrogen per 4 milliliters of serum.

Patients with chronic lymphatic leukemia and with multiple myeloma exhibited little or no antibody formation. Subjects with chronic myeloid leukemia showed antibody responses somewhat higher than the normal level. Individuals with acute leukemia demonstrated the production of exceedingly large amounts of antibody, and this response was impaired in patients treated with cortisone. 
The ability to form antibody to pneumococcus capsular polysaccharides is influenced by the type of leukemia present. Individuals with the highest percentage of immature white blood cells in their peripheral blood and bone marrow had the largest antibody responses. There was no apparent relation between the total white blood cell count in the peripheral blood and the amount of antibody produced. Patients with acute leukemia were noted to have a distinct impairment of antibody response when receiving cortisone.

Some Hormonal Aspects of Water Excretion in Man. Alexander Leaf, H. Rasmussen, A. R. Mamby and M. S. RABEN, Boston, Mass. (Introduced by Walter Bauer).

It has been claimed that anterior pituitary destruction will ameliorate or abolish experimental diabetes insipidus. A patient with diabetes insipidus was observed to lose polyuria and polydipsia spontaneously. Studies revealed insufficiency of anterior and posterior pituitary function. However, the physiologic defect of diabetes insipidus, inability to produce a hypertonic urine during severe dehydration, persisted. The decrease in polyuria reflected simply a decreased total solute load requiring excretion.

Metabolic studies were conducted while the subject was kept on a constant diet. Salt and urea administration caused return of polyuria by increasing the excretory solute load. Thyroxine, desoxycorticosterone, cortisone, corticotrophin (Astwood), and growth hormone (Raben) had no effect upon the renal concentrating ability or water excretion. Hence their alleged diuretic actions are not direct effects but are secondary to their action of increasing metabolism, appetite and the urinary solute load.

The inulin and PAH clearances of this subject were $50 \%$ of normal, consistent with panhypopituitarism. With corticotrophin these clearances rose to $75 \%$ of normal. Growth hormone, which has been considered to be the renotropic hormone, failed to elevate the renal clearances above the control level when given for twelve days in dosages up to $240 \mathrm{mgs}$. per day. Balance studies revealed that this absence of effect of growth hormone on renal clearances is to be contrasted with the phosphorous and nitrogen retention that occurred during its administration. These observations with an anabolically active preparation of growth hormone suggests that the renotropic effects of the anterior pituitary are not caused by growth hormone.

Protection of Tuberculin Hypersensitive Cells from Toxic Action of PPD by Cortisone in Tissue Culture. RoBert H. Leahy and Herbert R. Morgan,* Rochester, N. $Y$.

Previous investigators have demonstrated that ACTH or cortisone will inhibit the development of the lesions in experimental allergic diseases in animals; this might be due to suppression of : a) humoral antibody formation, b) tissue response to injury, or c) damage to capillaries which accompanies such reactions. It was decided to investigate the role of the response of isolated tissues in culture where the factors of humoral antibody and capillary damage are excluded. Splenic tissue from tuberculin hypersensitive guinea pigs was selected for these experiments, since Rich had demonstrated the cytotoxic action of tuberculin upon macrophages from such tissue.

The roller tube method in tissue culture was used with a fluid medium to which was added: 1) cortisone acetate suspension, or 2) PPD, or 3) both cortisone acetate and PPD. For the first 24 hours culture tubes received plain nutrient medium or the cortisone-containing medium, depending on their subsequent experimental conditions. These media were replaced by nutrient containing PPD or nutrient containing both PPD and cortisone. Daily measurements of migration of macrophages from the splenic fragments were made. Tuberculous tissue grown in nutrient containing PPD alone produced few macrophages, which failed to migrate after the first day and appeared to undergo cytolysis. Tuberculous tissue grown in nutrient containing both cortisone and PPD showed migration and numbers of macrophages equal to that seen with tissue grown in nutrient alone and gave no evidence of cytolysis.

These findings indicate that cortisone acts directly on tuberculin hypersensitive cells in vitro to protect them from the cytotoxic action of PPD and therefore that the suppression of humoral antibody formation or protective action against damage to capillaries is not essential for the influence of cortisone on certain aspects of cellular injury in hypersensitivity.

The Relation of Tissue to Serum Acid Phosphatase Concentration in Diagnosis and Treatment of Metastatic Adenocarcinoma. H. M. Lemon, I. Asimov, M. M. Davison, M. K. Schwartz and M. A. Derow, Boston, Mass. (Introduced by C. P. Emerson, Jr.).

Acid phosphatase is a universal constituent of cell nuclei, but varies widely in concentration in cytoplasm, where large amounts are present only in adult prostatic glands, neurones, certain endocrine glands, and keratinizing epidermis. Quantitative histochemical estimations of acid phosphatase activity of 54 human carcinomas and sarcomas and their normal homologous tissues of origin indicate increased concentrations of enzyme in adenocarcinomas of breast and colon, but not in epidermoid carcinomas. Forty-four observations of 22 colon carcinomas using a previously described technic (Science 109: 233-235, 1949) showed a mean of $0.145 \pm 0.014$ (S.E.) mg. $\mathbf{P}$ hydrolyzed at $\mathrm{pH} \mathbf{5 . 0}$ from glycerophosphate per mg. cellular nitrogen per hour, compared with $0.092 \pm$ 0.007 (S.E.) mg. for homologous colon mucosa. Benign uterine tumors and chronic inflammatory lesions did not show any deviation of phosphatase activity from normal. Partial purification of acid phosphatase, yielding a preparation with 3 major electrophoretic components, has been accomplished using prostatic tissue, and an extensive comparison has been made of the enzymatic function of 
acid phosphatase from benign hyperplastic and malignant sources.

These tissue observations supplement those made previously on the prostate by Huggins and others, and have an important bearing upon the use of serum acid phosphatase determination in the differential diagnosis of osteolytic bone disease and in evaluation of effects of hormone therapy of neoplasia. This will be illustrated by examples taken from a series of over 30 patients with advanced metastatic adenocarcinoma observed over a period of several years. Since acid phosphatase remains juxta-chromosomal within the nucleus during carcinogenesis, frequently appears to fluctuate in concentration within cells and body fluids according to tumor growth rate and nature of endocrine therapy and has been shown in this work to hydrolyze phosphorylated amino acids, further investigation appears indicated of its relation to malignant cellular degeneration.

Metabolic Fate of Infused Erythrocytes. Stanley M. Levenson,* Mary A. Maloney, Elizabeth A. Lounds and James L. Conkin, Richmond, Virginia.

Blood transfusions have been recommended for the nutritional therapy of the sick or injured. The view that infused erythrocytes are a particularly good source of nutritive protein has been based on observations indicating little increase in nitrogen excretion following such infusions. Retention has been arbitrarily equated with utilization. Data contrary to this viewpoint have been obtained in patients in anabolic and catabolic nutritional states.

Patients with various types of serious injury or illness were maintained on constant oral dietary intakes. After suitable control periods, serologically identifiable erythrocytes were infused. Observations were made of body weight, nitrogen, sodium, and potassium balances, plasma and red cell volumes, erythrocyte survival, bone marrow morphology, and various liver functions. The apparent positive nitrogen balance immediately following the red cell infusions does not connote rapid metabolic utilization of the red cell protein. Rather, the rise in urinary nitrogen is slight because the red cell nitrogen participates directly in the body metabolic pool only over a period of many weeks. In no instance was the survival of the infused cells markedly shortened. A limited amount of nitrogenous compounds is made available indirectly through an associated depression of erythropoiesis. The decrease in red cell production is proportional to the quantity of red cells infused. Once the period of relative increase is over, erythropoiesis proceeds normally. The quantity of nitrogen which might be available as a result of depressed erythropoiesis even if complete is small, and only a similar relatively small quantity can be expected from the normal breakdown of infused cells. Erythrocytes, once anemia has been corrected, should not be infused for "feeding purposes"-the small contribution to the nutritional requirements of the patient does not warrant the associated risks.
The Relation of Stroke Output to Left Ventricular Filling Pressure in Normals and in Patients with Cardiovascular Disease. Benjamin N. Lewis, Frorence W. Haynes, Hector E. J. Houssay and Lewis Dexter,* Boston, Mass.

Use of pulmonary "capillary" pressure as an index of left auricular pressure has made possible the study of left ventricular function in man. The relation of stroke output to filling pressure of the left ventricle has been studied at rest and during brief exercise in seven normal persons and in 16 individuals with hypertension, coronary disease, or lesions of the aortic valve. In four of seven normals there was slight increase of pulmonary "capillary" pressure on exercise with a concomitant increase of stroke output. In seven patients with mild manifestations of cardiovascular disease pulmonary "capillary" pressure was normal at rest, but increased above the normal range on exercise and this increase was all in cases associated with an increase of stroke output. In 8 of 9 individuals with moderate to severe signs and symptoms of congestive heart failure in whom pulmonary "capillary" pressure was abnormal at rest, further increases of pressure on exertion failed to produce any significant increase in stroke output. The increase of stroke volume resulting from increased diastolic fiber length of heart muscle and, therefore, from increased filling pressure (Starling's law) is only one of several mechanisms by which the normal heart can increase its minute output to meet increased bodily needs. This mechanism is, therefore, not demonstrable in all normal subjects. In patients with mild cardiovascular impairment, increase of filling pressure and therefore of diastolic size was apparently an important mechanism in increasing output during the stress of exercise and was demonstrable in all individuals studied. In those with more advanced symptoms in whom left ventricular filling pressure was already abnormal at rest, increased diastolic pressure was already optimal or nearly so for these diseased hearts, since further increases of filling pressure on exercise produced no increase in stroke output.

\section{Reflex Vagal Action on the Heart in Man Produced by} Ischemic Stimulation of the Carotid Body. ARTHUR J. Linenthal, Boston, Mass. (Introduced by Herrman L. Blumgart).

Anoxic chemoreceptor stimulation, of recognized importance in the reflex control of respiration, has not heretofore been described as a cause of reflex vagal effects on the heart in man. We have found that constant, continued pressure over the carotid artery bifurcation, in normal subjects as well as in cardiac patients, produces striking vagal action on the heart and marked respiratory stimulation, with or without a preceding carotid sinus pressoreceptor cardiac reflex. Changes in consecutive cycle lengths, the most sensitive cardiac vagal effect, were measured from electrocardiograms; changes in respiratory rate and volume were determined from pneumograms. These cardiac and respiratory reflexes are attributed to ischemic (anoxic) stimulation of the carotid body.

The carotid body cardiac reflex is of vagal origin: 
cycle length increases; respiratory sinus arrhythmia appears or is exaggerated, with particular prolongation of the expiratory cardiac cycle; atropine blocks these effects without influencing the respiratory changes. This reflex action appears after 20 to 40 seconds of pressure, becomes maximal in about 60 seconds, and may then show slow, cyclic variations in intensity. The simultaneous increase in respiratory rate and volume is not the cause of the carotid body cardiac effect: similar voluntary respiratory changes produce decreased rather than increased cycle lengths. The vagal cardiac effect of continued carotid sinus stimulation, on the other hand, is maximal in the first few seconds of pressure, returns to the control level after 10 to 20 seconds, and is unaccompanied by respiratory changes.

Stimulation of chemoreceptors in the carotid body and elsewhere may contribute to the development of the frequent and often serious disturbances of cardiac rate and rhythm which occur during conditions of systemic anoxia. The hitherto unexplained association of cardio-vagal and respiratory stimulation during Cheyne-Stokes hyperpnea may also involve this reflex mechanism.

The Role of Carbon Dioxide Tension in the Pathogenesis of Pulmonary Hypertension. FRANK W. LOVEJOY, JR., Paul N. G. Yu, R. E. Nye, JR. and H. A. Joos, Rochester, N. Y. (Introduced by W. S. McCann).

A statistical analysis of the relationship of mean pulmonary arterial pressure to possible determinants has been made in 46 patients, 33 of whom had chronic pulmonary disease. Patients with mitral stenosis or congenital heart disease were excluded. A significant relationship was found between pulmonary arterial pressure and (a) the $\mathrm{pco}_{2}$ of mixed venous blood, (b) $\mathrm{pCO}_{2}$ of arterial blood, (c) the degree of emphysema as expressed in terms of per cent residual air volume (\% of the total capacity), and (d) the degree of oxygen saturation of arterial blood.

The hemodynamic effects of $\mathrm{CO}_{2}$ added to inspired air during cardiac catheterization have been studied in a group of 30 patients. Complete data were available in 11 patients breathing $3.6 \% \mathrm{CO}_{2}$ and in ten patients breathing $6 \% \mathrm{CO}_{2}$. Analysis of these cases revealed that a rise in mean pulmonary artery pressure and pulmonary arterial resistance occurred in both groups, but was proportionately greater in the group breathing $6 \% \mathrm{CO}_{2}$. Neither anoxia nor increased blood flow was responsible for these changes. In general, the patients with most emphysema showed the greatest rise in pulmonary arterial pressure. Two normal subjects showed a fall in pulmonary arterial pressure when breathing $6 \% \quad \mathrm{CO}_{2}$. The implication of these findings is discussed.

Effects of Alterations of Body Potassium on Digitalis Toxicity. Bernard Lown, John M. Weller, Norman Wyatt, Rolf Hoigine and John P. Merrill, Boston, Mass. (Introduced by Samuel A. Levine).

Utilizing acetyl strophanthidin, an ultra-rapid digitalislike body, and the Kolff-type of artificial kidney, a method has been evolved for studying the effects of selective electrolyte alterations on the action of digitalis in the intact animal.

The relation of body potassium to the toxic action of digitalis has been explored in seven dogs. Control digitalizations given every two hours without evidence of cumulation were carried out prior to dialysis and during dialysis against a bath with a potassium of $4 \mathrm{mEq} / \mathrm{L}$. Similar digitalizations were carried out during dialysis against baths containing zero and $8 \mathrm{mEq} / \mathrm{L}$ potassium. The toxic end points for digitalization were ventricular premature beats or ventricular tachycardia.

Potassium extraction during two hours dialysis averaged $26 \mathrm{mEq}$. per animal, lowered serum potassium to $2.3 \mathrm{mEq} / \mathrm{L}$ and resulted in: 1) A $60 \%$ decrease in dosage of acetyl strophanthidin necessary for toxicity. 2) A five to tenfold increase in duration of toxicity. 3) Toxicity consisting of bigeminal rhythms or alternative bidirectional tachycardias not observed without potassium depletion. Continued potassium extraction while the serum level was maintained constant resulted in accentuation of the above changes.

Potassium addition raising the serum level to an average of $6.5 \mathrm{mEq} / \mathrm{L}$ resulted in: 1) A $50 \%$ increase in dosage of acetyl strophanthidin for toxicity. 2) Toxicity characterized by marked transiency of ventricular irritability with suppression of ventricular but not auricular manifestations of digitalis overdosage.

\section{Determination of Estrogen Excretion During Chorionic Gonadotrophin Therapy as a Test of Human Leydig Cell Function. William O. Maddock, Detroit, Michi- gan, and Warren O. Nelson, Iowa City, Iowa. (In- troduced by Gordon B. Myers).}

Biological assay of urinary estrogens has proved a reliable and sensitive measure of Leydig cell response to chorionic gonadotrophin as indicated by the following observations. The administration of 5,000 units of chorionic gonadotrophin three times weekly for six or more weeks produced a five- to twenty-fold increase of urinary estrogens in each of seven men having functioning testes, but no increase in three men lacking testes. Urinary estrogens rose within twenty-four hours after initiating therapy and remained elevated throughout treatment. It was concluded that estrogens originated from Leydig cells since repeat testicular biopsies obtained from four patients during therapy revealed a marked increase of Leydig cells but regressive changes in seminiferous tubules. In contrast to the striking and sustained elevation of estrogen excretion, the rise in urinary 17 -ketosteroids was less marked (approximately two-fold) and was more variable, individual values during therapy often falling within the pretreatment range.

In each of three hypogonadotrophic eunuchoids and in three of five men having adult hypopituitarism, initially low estrogen levels increased markedly during chorionic gonadotrophin therapy. Pretreatment testicular biopsies from the six responsive patients revealed inactive structures which appeared capable of being stimulated. The 
two unresponsive patients had completely hyalinized testes. Biopsies obtained from two of the eunuchoids after three months of therapy revealed growth and development of Leydig cells.

Determination of urinary estrogens before and following a standard dose of chorionic gonadotrophin is therefore suggested as a test of Leydig cell function, analogous to the test of adrenal cortical function in which 17ketosteroid determinations are used to evaluate response to ACTH.

The Effect of Body Position on Regional Alveolar Air Concentrations. C. J. Martin, F. Cline, JR. and $\mathrm{H}$. Marshall, Seattle, Washington. (Introduced by $R$. H. Williams).

Unequal ventilation of the lungs has been measured at mouth level by various techniques. We have measured end-expiratory alveolar oxygen and carbon dioxide concentrations from upper and lower lobe bronchi in man and determined the effect of body position upon these concentrations. Twenty-one normal volunteers under basal conditions were given phenobarbital $(0.1 \mathrm{gm}$.) and atropine ( $0.41 \mathrm{mg}$.) as premedication. The pharynx and larynx were anesthetized with cocaine $(5 \%)$ in epinephrine $(1: 4000)$. Radio-opaque plastic (2 mm. O.D.) catheters were guided into the trachea, and under fluoroscopy into the desired lobar bronchus. Simultaneous end-expiratory gas samples were obtained from the right upper and right lower lobe bronchi with the subject in the supine, upright and Trendelenburg positions. Duplicate gas analysis was conducted in a Scholander $0.5 \mathrm{cc}$ gas analyzer. After all samples were obtained a small amount of contrast mixture was instilled through each catheter and films taken.

In the supine position the right upper lobe alveolar oxygen values are below those of the right lower lobe by approximately one volume per cent. The difference is not statistically significant $(p=0.02)$. In the upright position the right upper lobe oxygen values are above lower lobe values by approximately two volumes per cent. The difference is statistically significant $(p=0.001)$. The direction of change in carbon dioxide values is opposite to that of oxygen. In the Trendelenburg position no significant difference has been found between simultaneous upper and lower lobe samples.

Lobar differences in alveolar gas concentrations exist. Particularly significant is the higher oxygen concentration in the upper lobe as compared with lower lobe when the subject is upright. This difference is of interest in relation to current theories explaining apical localization of adult pulmonary tuberculosis. The higher oxygen values in the upper lobe when upright would seem to favor growth of the tubercle bacillus.

The Mechanism of Protoveratrine-Induced Oliguria. Edward Meilman, Boston, Mass. (Introduced by Joseph E. F. Riseman).

The lowered blood pressure induced by intravenously administered protoveratrine in persons with hypertension is attended by a marked diminution of urine volume. The oliguria is accompanied by a striking diminution of sodium and chloride excretion which differentiates the process from oliguria due to anti-diuretic hormone. Potassium excretion during this time diminishes but slightly. That active absorption of water from the glomerular filtrate is involved is indicated by the marked increase in concentration of inulin or paraaminohippurate. Although the hypotension may cause acutely a significant decrease in glomerular filtration rate and in effective renal plasma flow, these may return to control values without increasing the excretion of sodium or the volume of urine. The duration of this effect is about four hours. Thereafter, despite a continued low level of blood pressure, urine volume and sodium excretion rise. Continuous hydration causes an increase in urine volume before this time, but the diminution in sodium excretion is not affected.

Forty to sixty minutes after intravenous injection of a mercurial-theophylline complex (mercuhydrin) during the period of oliguria, a very marked rise in the output of both sodium and water results.

These observations indicate that either the acute hypotension, or the acute fall in GFR or ERPF, initiates a marked tubular absorption of sodium and chloride, but not potassium, and that water is concurrently osmotically resorbed. This process persists despite recovery of both GFR and ERPF to control values. The fact that mercury overcomes this effect suggests that this resorption of sodium may be mediated by a tubular sulfhydryl enzyme system.

Occurrence of Hepatitis in Cortisone-Treated Rats Following Inoculation with Human Infectious Hepatitis Serum. George W. Melcher, JR., J. W. Blunt and Charles Ragan,* New York, N. Y.

Rats made hyperadrenal by the administration of cortisone acetate were inoculated subcutaneously with human serum obtained from an individual with viral hepatitis. This serum (obtained from Dr. Donal R. Fitch) had previously been tested on human volunteers and had been proven capable of producing the clinical picture of infectious hepatitis.

Diffuse focal hepatitis was observed microscopically in the livers of these rats. Subsequent inoculation of a suspension prepared from the livers of these animals produced hepatitis in a second group of cortisone-treated rats.

No hepatitis has been observed in the following groups of control animals: (a) normal rats given the same hepatitis serum or a liver suspension prepared from the rats showing liver lesions, (b) hyperadrenal rats given normal human serum or a suspension of normal rat liver.

Decreased Circulation in the Great Toe as the Earliest Manifestation of Vascular Disease in Diabetes Mellitus. Milton Mendlowitz,* Edward B. Grossman and Samuel AlPert, New York, N. Y.

The development of vascular disease in a group of 40 young males with diabetes mellitus was investigated by 
the measurement of blood flow in the great toe calorimetrically after release of sympathetic nerve tone by indirect heating supplemented by the intravenous administration of $5 \mathrm{mg} / \mathrm{Kg}$ of tetraethylammonium chloride. All of these patients had palpable pedal pulses, normal oscillometric tests and no overt evidence of renal or cardiac impairment. When the occasional cases with calcification of the arteries as demonstrated by roentgenograms of the legs or with retinal changes were excluded, it was found that approximately one-fourth of the remaining cases revealed blood flow values below the lower limit of the normal range on repeated testing. Statistical evaluation of the difference between the diabetic and a normal control group consisting of 30 patients also repeatedly tested revealed a significance ratio greater than 3.0. No correlation could be established between the decreased hallucal circulation and the cholesterol-phospholipid ratio. There was also no significant correlation between the apparent duration, severity or adequacy of control of the diabetes and the circulatory changes. None of these patients had known diabetes for more than ten years or was under the type of control which could be classified as excellent.

\section{Renal Damage in Rats Fed Large Quantities of Sodium} Chloride. George R. Meneely, Robert G. Tucker, Stewart H. Auerbach and William J. Darby,* Nashville, Tennessee.

Six groups of thirty male Sprague-Dawley rats five weeks old were placed on purified diets complete and identical except for sodium chloride. The control group received $0.15 \%$ sodium chloride in the diet. The five experimental groups received progressively higher proportions of sodium chloride: $2.8,5.6,7.0,8.4$ and $9.8 \%$, respectively. The diets and demineralized water were available ad libitum. When first placed on the experimental diets, marked polydipsia and polyuria occurred. After three days these rats appeared to be severely dehydrated. Two weeks later the rats all looked healthy and were gaining weight regularly.

During the third and fourth months edema developed in $9 \%$ of the rats eating the three highest levels of sodium chloride. Sudden weight gains of 40 to 160 grams occurred. Extracellular water measured with radioactive sodium rose from the control level of $25 \%$ to $57 \%$ of body weight. These edematous animals were severely anemic and showed lipemia and hypoproteinemia. At autopsy there were obvious edema and anemia. Otherwise, microscopically, all the tissues except the kidneys appeared normal. In relatively bloodless glomerular tufts there were foam cells containing large amounts of fat staining material. The tubules exhibited a nephrotic degeneration and contained also much fat staining material. There were lesser deposits of lipoid in some of the arterioles.

Three edematous animals survived this phase, rapidly lost their extra fluid and became extremely emaciated and cachectic. Again, all tissues seemed essentially normal except for a renal lesion similar to that described above but more extensive. Many glomerular tufts were obliterated. Tubular degeneration was extreme. No evidence of adrenal failure was found.

\section{Psychological and Somatic Factors in Coronary Artery} Disease. Henry H. W. Miles, Samuel Waldfogel and Edna L. Barrabee, Boston Massachusetts. (Introduced by Stanley Cobb).

Psychiatric interviews, a detailed social inventory and a battery of psychological tests were carried out upon a group of $\mathbf{4 5}$ men with uncomplicated coronary artery disease. Forty-eight healthy control subjects, 20 of whom were carefully matched with 20 of the coronary patients with respect to age, somatotype, ethnic origin and socioeconomic background, were similarly studied. All subjects had been previously investigated with great care so that data concerning past medical history, anthropological measurements and blood chemistry were available.

After consolidating the information gathered by various means, we summarized for each individual such factors as family relationships, characteristic patterns of reaction to the stresses of life, characteristic personality traits and ego defenses and (in the 45 coronary patients) the specific setting in which the illness became manifest.

The two groups were compared in terms of personality factors and also in terms of other factors such as family incidence of cardiovascular disease, history of physical stress and strain, somatotype and blood lipid levels.

The discussion is developed in the frame of reference of the concept of multiple causality. Those characteristics of the individual as well as those factors of the environment which seem to be relevant and causal are considered, with some tentative interpretations of the correlations found by us and by other investigators.

Fructose Metabolism in Diabetic Subjects. Max MILLER,* James W. Craig, Hiram Woodward, Jr., J. Evan Owens and William R. Drucker, Cleveland, Ohio.

Previous work from this laboratory has shown that the intravenous administration of fructose to diabetic subjects in the absence of exogenous insulin produced the same blood pyruvic acid rise as in normal subjects, while glucose produced a slight or delayed rise. The blood fructose disappearance curve and the amount of urinary fructose were the same as in normals. Serum inorganic phosphorus fell after fructose but not after glucose. Hepatic vein catheterization studies showed that the splanchic uptake of fructose was similar in diabetic and normal subjects and was greater than the uptake of glucose.

Others have stated that the apparent advantage of fructose over glucose in the diabetic was lost if the oral administration of fructose was continued for several days. Two diabetic patients were given 50 and 100 grams of fructose, respectively, each morning without insulin. No increase in fructose excretin or change in the fructose tolerance curve was noted during 9 days of either oral or intravenous administration. At the end of the periods fructose still retained its advantage over glucose, as 
measured also by total hexose excretion, rise in blood pyruvate and fall in phosphorus.

Two cases of diabetic acidosis have been treated with fructose, saline and insulin and the results compared with cases in 2 previous series treated with glucose, saline and insulin, and with saline and insulin alone. The administration of 175 grams of fructose during the initial 6 hours resulted in the excretion of only 7 and 3 grams, respectively, and blood fructose levels did not exceed $100 \mathrm{mg} . \%$. Blood glucose levels fell approximately at the same rate as in the series treated with saline and insulin alone. Falls in blood ketone levels, water balance data, and changes in blood pyruvate in the 3 series will be discussed.

Phospholipid Turnover in Man. Hugo W. Moser, Boston, Mass. (Introduced by Kendall Emerson, Jr.).

A method has been developed which makes it possible to calculate the per cent of plasma phospholipid turned over per unit time. Following the intravenous injection of a tracer dose of carrier-free inorganic $P_{a z}$, the specific activity of plasma lipid and urinary phosphorus is followed for 96 to 120 hours. We have confirmed Handler's findings that the specific activity of urine phosphorus is almost identical with that of plasma inorganic phosphate. When urine phosphate is plotted against time it can be expressed as the sum of two exponentials.

The data obtained indicate that the inorganic phosphate of the urine is a closely approximate measure of the immediate precursor of lipid phosphorus, according to the criteria of Zilversmit. Knowing the simultaneous rates of change of the specific activity of lipid phosphorus and its immediate precursor an equation can be derived for the calculation of the actual percentage turnover of plasma phospholipid per unit time. Since Chaikoff has shown that plasma and liver phospholipid are in equilibrium and form a single pool, we are presumably measuring the turnover rate in this pool. In three normal subjects from 1.4 to 1.9 per cent of phospholipids were turned over per hour, whereas in three nephrotics this value was between 0.6 and 0.8 per cent. In a patient with myxedema 0.6 per cent was turned over per hour before treatment and 1.1 per cent during therapy with thyroid.

In these patients the plasma phospholipids were elevated to the same extent that their phospholipid turnover rate was depressed, as compared to the normal subjects. The absolute quantity of plasma phospholipid renewed per unit time, therefore, was the same in the normal and pathological subjects.

Preliminary Studies on the Purine and Pyrimidine Bases of Human Bone Marrow as Determined by Paper Chromatography. I. Variations in Pernicious Anemia in Response to Therapy. JOHN F. MUELLER, HeLEN Stix Glazer and Richard W. Vilter,* Cincinnati, Ohio.

Numerous biologic studies have linked folic acid and vitamin $B_{18}$ to the formation of pyrimidines and purines. Responses of patients with pernicious anemia to thymine and to a lesser extent to uracil suggest a similar relation- ship. Since thymine is present only in desoxyribonucleic acid, an abnormality in this substance might be expected; however, cytochemical and spectrophotofluorometric techniques have suggested some defect in ribonucleic acid metabolism. It seemed advisable, therefore, to measure the qualitative and quantitative changes in the purine and pyrimidine bases of the bone marrow and apply this method to a study of pernicious anemia before and after specific therapy.

The buffy coat from a bone marrow aspirate is treated with cold trichloracetic acid. Lipids are extracted with hot ether-alcohol and the residue hydrolyzed with perchloric acid. The purines and pyrimidines are separated by paper chromatography with butyl alcohol as solvent. An alkaline pH separates guanine, cytosine, thymine and a mixture of adenine-uracil. An acid medium allows separation of thymine and uracil. The spots containing the various bases are detected in ultraviolet light, photographed, cut out and eluted in weak hydrochloric acid. Amounts in the eluate are determined in the Beckman spectrophotometer and expressed as molar ratios.

Bone marrows from normal persons have been studied and have contained all the known bases. Uracil is present in small amounts. In three of four patients with pernicious anemia in relapse studied so far the following observations have been made. (1) Thymine is low and gradually increases after therapy when determined on alkaline strips. (2) The ratio of uracil to thymine determined on acid strips decreases ten-fold after therapy suggesting that thymine increases as uracil decreases. These studies point to a fundamental defect in pyrimidines in pernicious anemia which is corrected by folic acid and vitamin $\mathrm{B}_{\mathbf{1 2}}$.

The Effects of Cortisone and ACTH on Splanchnic Circulation and Metabolism. J. D. MYers * and W. Jape Taylor, Durham, North Caroline.

The profound metabolic effects of cortisone and ACTH might be expected to be associated with alterations in hepatic circulation and splanchnic metabolism of oxygen and glucose. The hepatic blood flow (estimated by hepatic venous catheterization and the bromsulphalein tecnic of Bradley) and splanchnic balances of oxygen and glucose have been measured in a) 8 human subjects, with only minor ailments, who received $200 \mathrm{mg}$. of cortisone orally approximately four hours before study, and b) 12 persons with illnesses which required ACTH. Each patient on ACTH served as his own control, the observations made after one day to three weeks on ACTH being compared to control studies done before or after the period of treatment. No pre- or post-treatment control data were collected on the cortisone patients; their findings are compared with data from 62 similar but untreated persons.

Cortisone resulted in a mean increase in hepatic blood flow of 43 per cent $(p<0.01)$. Splanchnic oxygen consumption was augmented by 26 per cent $(p<0.01)$ hepatic arterio-venous oxygen difference decreasing only slightly and insignificantly. Cortisone produced no measurable 
alterations in arterial blood glucose concentration, hepatic A-V glucose difference, or splanchnic glucose production.

The effects of ACTH on hepatic blood flow and splanchnic oxygen consumption depended upon the control levels of those factors. Five of the 12 subjects had elevated control flows and oxygen consumptions presumably due to their diseases, and $\mathrm{ACTH}$ resulted in no further augmentation. The other 7 subjects, with normal control values, showed a mean increase in flow of 28 per cent $(p<0.01)$ and in oxygen consumption of 26 per cent $(p<0.05)$. Again hepatic A-V oxygen difference was unchanged. Three of the subjects on ACTH acquired hyperglycemia and several showed rises in splanchnic glucose production; the two phenomena were not related, however. Neither drug affected BSP clearance.

In summary, both cortisone and ACTH appear to raise hepatic blood flow and splanchnic oxygen consumption above the usual basal levels.

Demonstration of Existence of Long Term "Asymptomatic" Blood Carriers of Hepatitis Virus with Confirmation in Human Volunteers: Relationship of Carriers to Acute Viral Hepatitis in Whole Blood Recipients. JOHN R. NeEFE,* ROBERT F. NORRIS, JOHN G. Reinhold, John W. Oliphant, Donald R. Fitch, Richard K. Watanabe, Charles B. Mitchell and David S. Howell, Philadelphia, Pa.

During the past 18 months, 15 patients with acute viral hepatitis, presumably acquired from whole blood transfusions, have been observed. Nine of the patients had had only a single whole blood transfusion, four had 2 or 3 transfusions each and the remaining three had multiple transfusions. All of the donors involved with 12 of the cases became available for careful study which included complete history, physical examination, and a comprehensive group of hepatic tests. Needle biopsies of the liver were obtained on 3 of the donors. Serum specimens for transmission experiments in human volunteers were obtained at intervals from the suspected donors. None of the donors had histories of jaundice or hepatitis and all met the usual criteria required by most large blood collecting centers.

Definite evidence of hepatic disturbance (histologically confirmed in 3) was demonstrated in the blood donors for 10 of the 12 patients with acute viral hepatitis. Serum specimens obtained from 5 of the suspected donors three to twelve months after the time of their original infectious donations subsequently were tested for infectivity in human volunteers. The development of hepatitis with jaundice in 4 of the 5 groups of volunteers inoculated with the 5 respective sera demonstrated conclusively: (1) That the suspected donors were the sources of infection of the original patient recipients and (2) that 4 of the 5 donors were "asymptomatic" blood carriers of hepatitis virus over a minimum period of 3 to 12 months.

The results appear to be of considerable significance in relation to the serious problem of blood transmitted hepatitis. Demonstration of the existence of "asympto- matic" blood carriers represents confirmation of a factor of major epidemiological importance, previously suspected but not proved. The findings to date suggest routine "hepatic" testing of donor blood might provide one additional means of decreasing the present serious incidence of blood transmitted viral hepatitis.

The Direct Measurement of the Extracellular Phase of Tissues. G. Nichols, JR., N. Nichols, W. B. WeIl and W. M. Wallace,* Boston, Massachusetts.

Measurements of the extracellular fluid in the human subject by inulin and thiosulfate give volume distributions so small that they are difficult to reconcile, biochemically or morphologically, with balance data and tissue analysis in experimental animals and man. To clarify this problem, the distribution of a single injection of inulin and thiosulfate was measured directly in the tissues of eight nephrectomized dogs, at varying time intervals, and compared with simultaneous electrolyte, water and collagen analyses of these tissues.

In all the tissues studied, the chloride space exceeded the thiosulfate space, and this exceeded the inulin space. Dense connective tissue, such as tendon, showed an increase in size of the thiosulfate and inulin spaces from 3 to 6 hours. At 6 hours thiosulfate space represented $75 \%$ of the extracellular phase ; inulin, $25 \%$. Thiosulfate space was $60 \%$ of chloride space in muscle, $75 \%$ in skin, and showed no increase from 3 to 6 hours. Inulin spaces in muscle and skin increased slightly from 3 to 6 hours, reaching $50 \%$ of the chloride space.

Since neither thiosulfate or inulin spaces reach the size of the plasma ultrafiltrate phase of tendon or skin in 6 hours, they are not accurate measures of the extracellular fluid within this time interval. However, from a morphologic point of view, inulin or thiosulfate space in tissues, plus that fraction of the connective tissue phase which remains unpenetrated at any given time, defines a space which is only slightly smaller than the space defined by chloride, suggesting that when chloride space is corrected for the higher concentration of chloride in connective tissue, it is both biochemically and morphologically the best measure of the extracellular space.

Hypertension and Pyridoxine Deficiency in the Rat. NoRman S. Olsen and William E. Martindale, Nashville, Tennessee. (Introduced by John B. Youmans).

Recent experimental observations indicate that certain types of hypertension may be of dietary origin. In the present report we have investigated the relation of elevated blood pressure to the vitamin $B_{6}$ group of compounds, which is known to be important in the metabolism of amino acids.

Pyridoxine deficiency was produced in young Wistar rats by means of a deficient diet supplemented by desoxypyridoxine. In addition to the typical clinical symptoms these animals exhibited an increase in blood pressure highly significant statistically as compared to litter-mate controls. A group of the hypertensive-deficient rats was placed on an adequate diet supplemented with pyridoxal. 
A rapid increase in weight together with a significant fall in blood pressure to normal levels was noted. These observations were duplicated in older animals.

Further studies on organ weights, oxygen uptake of tissue homogenates, vitamin $B_{0}$ assay of tissues and histological survey of tissues were carried out. There was a significant increase in the adrenal weights of the deficient animals which was paralleled by a hypertrophic trend in the ventricular mass. The oxygen uptake of kidney and liver homogenates from the deficient animals was found to be about 30 per cent less than in the control group. Vitamin $B_{6}$ assays on the same tissues showed changes in the same direction. The histological survey gave the general impression of no arterial or arteriolar disease and no significant irreversible disease process.

Mechanisms of Adjustment in the Cerebral Circulation Upon Assumption of the Upright Position. JoHN L. Patterson, JR., and James V. Warren,* Atlanta, Georgia.

Both the blood flow through the human brain and the arterial pressure at brain level fall when the upright position is assumed, but the percentile diminution in blood flow is considerably smaller.

Continuous oximetric determinations of changes in the cerebral arteriovenous oxygen difference were made during $65^{\circ}$ (head-up) tilting. Since the oxygen consumption remains stable in non-fainting subjects, the reciprocal of the arteriovenous oxygen difference will vary directly with the blood flow. Arterial, internal jugular and cerebrospinal fluid pressures were measured with strain gauges mounted at ear level.

In this series mean arterial pressure was $19.2 \mathrm{~mm} . \mathrm{Hg}$ (24.3\%) lower, and the venous pressure $7.3 \mathrm{~mm}$. $\mathrm{Hg}$ lower in the upright than in the recumbent position. Internal jugular pressure actually fell to slightly subatmospheric levels in most subjects. The value of $1 /$ $(A-V) O_{2}$ averaged $14 \%$ less in the upright position. Changes in cerebrospinal fluid pressure closely paralleled the changes in jugular pressure.

The percentile fall in net perfusion pressure (arterial minus internal jugular pressure) was actually only slightly greater than that in the cerebral blood flow, owing to the fall in venous pressure.

The changes in cerebrospinal fluid pressure probably counterbalanced the fall in intracranial venous pressure and capillary pressure and thus prevented compression of these vessels.

Because a siphon effect appeared possible, experiments with a brain model were done, using thin-walled rubber tubing to simulate the jugular vein. These indicated that collapsible tubes can function as the descending limb of a siphon. Potential vasodilator stimuli were present in the form of increased capillary $\mathrm{CO}_{2}$ tension and decreased $\mathrm{O}_{2}$ tension resulting from reduction in CBF with maintenance of normal metabolism.

These results do not require the assumption of any cerebral pressoreceptor-vasomotor mechanism, and suggest operation of the siphon principle in this and possibly other areas of the circulation.
Physiological Effects of Bilateral Adrenalectomy in Man. O. H. Pearson, * V. P. Hollander, C. D. West, W. F. Whitmore and H. T. RANDALl, New York, N. Y.

Bilateral adrenalectomy has been carried out in seventeen patients with advanced cancer during the past year. It has been possible to maintain these patients satisfactorily during and after surgery with cortisone as the only replacement therapy. Patients maintained for many months on oral cortisone (average dose $50 \mathrm{mgs}$ daily) failed to manifest symptoms or signs of adrenal insufficiency or of hypercorticism. Among the group of patients maintained on approximately 50 milligrams of cortisone daily, we have observed normal eosinophile counts, normal values for urinary 17-ketosteroids and formaldehydogenic steroids, growth of pubic hair, normal insulin tolerance, normal water tolerance, normal glucose tolerance, normal blood sugar after prolonged fasting, reduction of urinary sodium excretion on salt deprivation, and the ability to withstand moderate stress.

Abrupt withdrawal of oral cortisone was associated with the characteristic symptomatology of adrenal insufficiency which began within twenty-four hours and intensified so that by the fifth to eighth day the patient's status was critical. Alterations in water and electrolyte balance were characterized by prompt antidiuresis, hemodilution, hyponatremia and hypochloremia. A negative balance of sodium and chloride usually occurred but was not associated with loss in body weight, presumably due to water antidiuresis. Potassium retention and hyperkalemia did not occur. Restitution of cortisone therapy resulted in profound water diuresis and prompt clinical improvement. Sodium and chloride were retained and the serum levels of these electrolytes returned to normal.

The clinical and physiological changes which occurred on cortisone withdrawal from the adrenalectomized patient have been closely simulated by the administration of pitressin tannate while cortisone therapy was maintained.

These observations indicate that water intoxication is an important feature of the physiologic effects of adrenal insufficiency in man.

\section{A Syndrome Resembling Impending Hepatic Coma Pro-} duced in Patients with Cirrhosis of the Liver by the Administration of Certain Nitrogenous Substances. Gerald B. Phillips, Robert Schwartz, George J. Gabuzda, Jr. and Charles S. Davidson,* Boston, Mass.

A syndrome indistinguishable from impending hepatic coma and typically consisting of mental disturbances and a characteristic tremor and electroencephalographic pattern was produced in five of nine chronic alcoholics with advanced hepatic cirrhosis by the oral administration of large quantities of certain nitrogenous substances. The abnormal signs always reversed within one or two days after cessation of the administration of these substances, which were given during periods of from two to twentytwo days.

Two patients developed the syndrome when given an 
ammonium containing cation exchange resin and again when given ammonium chloride. The characteristic signs did not recur in one of these two patients during a comparable degree of acidosis induced by calcium chloride administration. A similar reaction was ${ }^{\circ}$ observed in a third patient during one course of di-ammonium citrate and three courses of urea, but not during a period of high dietary protein intake. However, the syndrome did appear in two other patients following an increase in dietary protein intake. Four other patients failed to show any untoward effect when given separate courses of one or more of these substances, including increased dietary protein.

Serial determinations of serum $\mathrm{pH}$, carbon-dioxide, sodium and potassium, and of blood nonprotein nitrogen failed to reveal alterations which correlated with the clinical changes. The blood ammonia content (diffusible alkali measured by the Conway microdiffusion technique) was often increased during clinical reactions, but the changes were not consistent.

Conclusions: A syndrome apparently identical with impending hepatic coma was produced in five patients with advanced hepatic cirrhosis by the oral administration of an ammonium containing cation exchange resin, ammonium chloride, diammonium citrate, urea, and large quantities of dietary protein.

The administration of these substances to patients with liver disease may by hazardous.

Effect of Thiocyanates on Sodium Excretion in Normotensives and Hypertensives. KERMIT L. PINES and George A. Perera,* New York, N. Y.

A depressor action of thiocyanates in essential hypertension has been described by many investigators, although the percentage of responsive patients has varied considerably in different clinics. Because of apparent relationships between electrolyte metabolism and arterial tension, balance studies were undertaken before and during thiocyanate administration in 21 normal subjects and 14 patients with uncomplicated essential hypertension.

Following at least a six-day control period on a constant dietary regimen, sodium or potassium thiocyanate was given by mouth in divided doses daily for four days with resultant peak serum levels in the 4-7 mgs. per 100 cc. range. The sodium content of the diet was varied widely in different individuals in both groups, and observations were made under both ambulatory and resting conditions. The average urinary sodium excretion per day for the final four days of the control period was compared to that during the four days on thiocyanate, appropriate corrections being made when the sodium salt was employed.

Following treatment, the average daily urine sodium rose 12 meq. (15 per cent) in the entire normotensive series, and 27 meq. (33 per cent) in the hypertensive group. Among the 21 normotensives were five subjects in whom mean urinary sodium levels during thiocyanate administration exceeded the dietary intake; in contrast, all the hypertensives lost sodium in greater amounts than that contained in their diet.

In view of the effects of rigid salt restriction in hypertensive disease, the possibility is raised that thiocyanates may exert a depressor effect through similar mechanisms. Furthermore, this study provides added evidence for a difference in metabolic response of hypertensives when compared to normals.

Clinical and Experimental Studies with Tritium. T. C. Prentice, W. Siri, N. I. Berlin and R. J. Parsons, Berkeley, California. (Introduced by John H. Lawrence).

The present study was undertaken to develop further the use of tritium $\left(\mathrm{H}^{3}\right)$ for routine body water measurement and to extend the investigation of total body water to a variety of disease states. It was necessary, in conjunction with the above, to evaluate several factors which could influence the accuracy of total body water determinations with tritium-labeled water. These were: (1) the time required for complete mixing throughout body water following intravenous administration of tritiumlabeled water; (2) the possible existence of a significant isotope effect due to the large mass increment of tritium; and (3) the magnitude of the error introduced by exchange of tritium with the solid constituents of the body.

Total body water determinations were carried out in 57 patients, 20 of whom were normal, the remaining 37 being patients with cardiac, liver, and blood abnormalities.

Mixing appeared to be "complete" in all patients within three hours. Comparison of total body water as measured simultaneously with tritium and antipyrine in 15 patients revealed the former to be consistently $2 \%$ of body weight greater than the latter. These results compared very closely with deuterium-antipyrine ratios found previously, thus making any tritium isotope effect unlikely. This $2 \%$ difference could be accounted for quantitatively by exchange of tritium with solid constituents, as measured in a group of rabbits.

No characteristic patterns of total body water were found in any given clinical group. It was concluded that wide variations in individual fat content were responsible for the surprising over-all uniformity in proportion of body water in subjects with and without abnormal accumulation of fluid.

The Wolff-Parkinson-White Syndrome. MYron PrInzmetal,* Rexford Kennamer, Eliot Corday, John A. Osborne, Joshua Fiemds and L. Allen Smith, Los Angeles, Calif.

Various types of the Wolff-Parkinson-White (WPW) aberration were produced experimentally in 25 dogs by applying a direct electrical current of subthreshold intensity to the A-V node. Stimulation of the same region of the A-V node, at different rates, resulted in several forms of supraventricular arrhythmias, which were identical lesion.

A new technique of high-speed cinematography has enabled the cardiac movements to be recorded simultane- 
ously with the electrocardiogram. By this means it was observed that a premature localized contraction in one ventricle was related to the premature portion of the $R$ wave.

In addition to the usual cogenital type, numerous examples of an acquired nodal type of WPW aberration have been observed clinically in patients with organic heart lesions involving the region of the $A-V$ node. The most common cause seems to be posterior myocardial infarction.

The hearts of three such patients with acquired nodal type of WPW syndrome were examined histologically. In two, myocardial infarction and fibrosis had involved the $A-V$ node, while in the third this region was invaded by a fibrocalcific lesion.

Experimentally, section of the bundle of $\mathrm{His}$ always prevented the production of WPW aberration. Therefore, the impulse must reach the ventricles through the normal conduction system. It is unnecessary to presume that abnormal conduction pathways, such as the bundle of Kent, are responsible.

Newcastle Disease in Man. Robert W. Quinn, Robert P. Hanson, John W. Brown,* Carl A. Brandly, Madison, Wisconsin.

Interest in Animal-Human disease relationships has been stimulated at the University of Wisconsin, by collaboration of departments in the Medical School and College of Agriculture. The occurrence in man of Newcastle Disease of Fowls has received attention recently. This report is concerned with the observations obtained during the course of three cases of infections which occurred in laboratory workers. Two individuals were accidentally exposed to Newcastle Disease virus by aerosol spray; one of these also accidentally punctured his skin with a needle contaminated with active virus. The third patient had washed contaminated glassware. Symptoms appeared in approximately 24 hours. The most prominent and constant clinical features were malaise, photophobia with conjunctivitis, sore throat with superficial ulcerations of buccal mucosa, and preauricular adenitis. The duration was about 7 days, the illness of 1 of the 3 individuals was very mild, almost inapparent.

Virus isolations were performed by inoculation of embryonated eggs, followed by hemagglutination-inhibition tests on allantoic fluids of infected embryos. Newcastle Disease virus was obtained from conjunctival sac washings, 2 cases, nasal secretions, 1 , throat washings, 2 , salivia, 1 , blood, 1 , and urine, 1 .

Newcastle Disease appeared in chickens in the United States in 1941, and spread rapidly to become a major disease problem of poultry in this country. Thirty-three cases of the disease in humans were reported through 1950. Newcastle Disease virus was isolated in 7 ; viremia was demonstrated in 1 . The observations of investigators in Minnesota during a recent outbreak in a poultry eviscerating plant will add information.
A notworthy feature of the present report is the isolation of Newcastle Disease virus from one patient with nearly inapparent infection.

Metabolic Effects of a New Growth Hormone Preparation. M. S. Raben, V. W. Westermeyer and A. LeAf, Boston, Massachusetts. (Introduced by E. B. Astwood).

Growth hormone was recovered and concentrated from the residual solution following the purification of corticotropin with oxycellulose. Glacial acetic acid extraction at $70^{\circ} \mathrm{C}$. and subsequent treatment in acid environment established conditions markedly different from those generally employed in the preparation of growth hormone. This concentrate was equal in growth-promoting activity to those of Wilhelmi and of $\mathrm{Li}$, and was virtually free of corticotropin, thyrotropin, gonadotropins, and posterior lobe principles, and contained only traces of luteotropin, adipokinin, and intermedin. Unlike any growth hormone preparation previously studied, it was found to evoke anabolic effects in man. Intramuscular injection caused retention of nitrogen and phosphorus in a 26-year-old woman with hypopituitarism. Despite a daily intake of only 7.9 grams of nitrogen and 727 milligrams of phosphorus, the average daily excretion during eleven days of increasing doses of growth hormone was 6.7 grams of nitrogen and 686 milligrams of phosphorus as compared with 7.8 grams and 912 milligrams during ten control days. Thirty milligrams per day appeared to be an effective dose. Neither glycosuria nor increase in fasting blood sugar concentration occurred with 240 milligrams per day for six days.

Studies with this preparation did not confirm the current view that the diabetogenic effect of pituitary extracts is due to growth hormone. Daily injections in adult dogs failed to produce glycosuria. Responsiveness of the animals to pituitary diabetogenic effect was demonstrated by the production of glycosuria with Armour growth hormone in one-quarter the dose. Glycosuria disappeared on changing from Armour material to the larger dose of the present preparation.

Dosimetric Considerations with Radioiodine. J. E. RALL, Charles E. Foster and Wendell Peacock, New York, New York. (Introduced by Rulon W. Rawson).

The dynamic distribution of 29 large doses of $I^{121}$ (50 to 300 millicuries) given to 24 patients with cancer of the thyroid and the effect of this amount of radiation on the organism have been studied. The only serious effect of this radiation was manifested by the hematopoietic tissues.

A satisfactory dose response curve was obtained by a plot of fall in lymphocytes against blood radiation calculated as the integrated blood concentrated plus gamma radiation from $\mathrm{I}^{1 \mathrm{~m}}$ localized in the tumor. Due to the marked variation between patients in the rates of turnover of iodine it was not possible to predict the effect of any given dose without prior tracer studies. Suitable 
study of a previous tracer dose of radioiodine however permitted fairly accurate estimation of the response to a large dose.

With the above data it has been possible to calculate the relative advantages of the 4-day, 8-day and the 13-day isotopes of iodine in producing a more favorable tumor blood radiation ratio.

Antibody Formation in Surviving Tissues. HeLEN M. RANNey and Irving M. London,* New York, N. Y.

This study is designed to investigate the participation of various tissues in antibody formation by determining the capacity of surviving tissues to form antibody. The technique which has been developed avoids the difficulty of determining whether antibody present in a tissue has been synthesized by that tissue or whether it has been transported to the tissue from its origin at another site. Rabbits were immunized for several weeks with a vaccine of killed pneumcocci of a single type. Twelve hours before the animals were sacrificed, they were passively immunized with an antiserum to a pneumococcus of a type different from that used for the active immunization. Slices of surviving tissues were prepared, serum containing antibodies to both types of pneumococci was added as inert carrier, and the preparations were incubated with $\mathrm{C}^{16}$-labeled glycine. The formation of antibody in vitro could be expected to utilize some of the labeled amino acid so that the newly formed antibody would be labeled isotopically whereas preformed antibody would not be labeled. After incubation, tissues and media were separated by centrifugation, and large amounts of non-isotopic glycine were added to the supernatant solutions which were then dialyzed against $0.85 \%$ sodium chloride. The passively administered antibody and the antibody formed on active immunization were isolated successively by addition of type-specific pneumococcus polysaccharides. The radioactivity of the dried specific precipitates was determined.

Significant radioactivity was found in the specific precipitates of actively formed antibody in spleen, bone marrow, lung, and liver. The absence of significant radioactivity in the specific precipitates of passively administered antibody indicates that non-specific adsorption or co-precipitation of isotopically labeled products does not account for the isotope activity observed in the actively formed antibody. Thus far, using only the pneumococcus as antigen, we have not observed antibody synthesis by heart or kidney tissue.

Pituitary Respiratory-Quotient Depressing Factor. LILLian Recant, St. Louis, Missouri. (Introduced by Thomas H. Hunter).

The nature and locus of action of the pituitary respiratory-quotient (R.Q.) depressing factor has been studied. In a Ringer-phosphate medium, the R.Q. of a normal fasted rat diaphragm is .68 . In a glucose medium the R.Q. rises to .83. The in vitro addition of certain anterior pituitary preparations to the medium depresses the glucose R.Q. to .68. This effect is not reversed by insulin in vitro. Of the preparations tested, crystalline growth hormone showed the greatest activity. Though similar in many properties, the R.Q. factor does not appear to be identical with the growth hormone since loss of R.Q. activity may occur without change in growth activity.

On the addition of glucose, hexosediphosphate, 3phosphoglyceric acid, pyruvate, or acetate, the diaphragm R.Q. increased to values approaching theory. Growth hormone ( 100 micrograms $/ \mathrm{ml}$. medium) inhibited the rise in R.Q. only in the case of glucose and hexosediphosphate. Although no decrease in glucose or hexosediphosphate uptake occurred, lactate production from hexosediphosphate was significantly decreased. These data suggest that the R.Q. factor inhibits the transformation of hexosediphosphate to pyruvate and lactate.

An R.Q. depressing factor has been demonstrated in the serum of fasted normal rats but is absent in fasted hypophysectomized and fed normal rats. Correlation of glycogen and serum factor levels suggests a carbohydrate conserving function for the R.Q. factor, compatible with the above noted site of action. Serum studies will be extended to include normal and diabetic patients.

The implications of these observations with respect to human physiology will be discussed.

The Metabolic Effects of Compound F Acetate in Man. arnold S. Relman and William B. Schwartz, Boston, Massachusetts. (Introduced by Chester S. Keefer).

Balance studies with 17-hydroxycorticosterone acetate (Compound $\mathrm{F}$ acetate) have been carried out in a small group of male patients with normal adrenal function and in two with hypoadrenalism. When given intramuscularly in doses of approximately $200 \mathrm{mgm} / \mathrm{day}$ Compound $\mathrm{F}$ acetate produced mininal effects on electrolyte metabolism. When given orally in doses of 200-300 $\mathrm{mgm} /$ day the steroid produced large positive balances of sodium and chloride for several days after which no further retention occurred. There was initially a marked negative potassium balanced which in a few days rapidly decreased despite continued treatment. There was a large sustained negative nitrogen balance which soon exceeded the daily loss of potassium as predicted from the usual $\mathrm{K}: \mathrm{N}$ ratio. Urinary excretion of ammonia and phosphorus was increased but there was no significant change in urine titratable acidity. The serum bicarbonate rose slightly and the serum potassium decreased slightly. Glucose tolerance became impaired but there was no glycosuria. There was a small rise in excretion of total organic acids. Following withdrawal of Compound $\mathrm{F}$ acetate there was a negative balance of sodium and a positive balance of potassium.

In one short-term preliminary experiment, rigid sodium restriction appeared to modify the effect of Compound $F$ acetate on excretion of postassium, phosphate, nitrogen and ammonia.

Serial determinations of $\mathrm{I}^{121}$ uptake by the thyroid and assays of urinary 17-ketosteroid excretion suggested that Compound $\mathrm{F}$ acetate depressed pituitary release of thy- 
rotrophin and corticotrophin. In hypoadrenal subjects administration of the steroid increased urinary 17-ketosteroid excretion.

The Intravenous ACTH Test. Albert E. Renold, Jose Garcia-Reyes and Dalton Jenkins, Boston, Mass. (Introduced by George W. Thorn).

The capacity of the adrenal cortex to respond to its physiological stimulus as determined by ACTH administration constitutes the most specific measure of adrenocortical reserve. Quantitative studies have demonstrated: (a) adrenocortical activation by intravenous infusion of a given dose of ACTH is directly proportional to duration of infusion; (b) stimulation obtained over a given time is directly proportional to ACTH dosage up to a critical amount. Based on the derived log doseresponse curve, an intravenous $\mathrm{ACTH}$ test has been standardized: ACTH, $20 \mathrm{I}$. U. in $500 \mathrm{ml}$. normal saline, or 5 per cent dextrose, infused for 8 hours on two successive days. Indices utilized in evaluating adrenal response are: (a) changes in circulating eosinophils, (b) 24-hour excretion of 17-ketosteroids.

In preliminary studies, one hundred normal subjects and patients without adrenal disease exhibited a mean eosinopenia of 97 per cent (range 80-100) and a mean 17-ketosteroid rise of $5.4 \mathrm{mg}$. per day (range 0.9 to 19 ). Only six subjects sustained an eosinopenia less than 90 per cent. The wide range of 17 -ketosteroid values necessitates the administration of ACTH on two successive days. Results of this test in 35 cases reveal a mean 17ketosteroid rise of $4.4 \mathrm{mg}$. per day on the first day and $8.7 \mathrm{mg}$. (range 4.7-16) on the second. Thirty Addisonians showed a mean rise in eosinophils of 0.6 per cent (range $-29-40$ ) and a mean 17-ketosteroid fall of $0.2 \mathrm{mg}$. (range $-2.9=+1.9$ ). Twelve patients following bilateral adrenalectomy exhibited a mean eosinopenia of 5 per cent (range $-26-+20$ ) and a mean 17-ketosteroid rise of $0.2 \mathrm{mg}$ per 24 hours (range $1.5-+2.9$ ).

The intravenous ACTH test is the method of choice in evaluating adrenocortical function because of: (a) minimal amounts of ACTH required; (b) maximal stimulation produced; and (c) elimination of the vagaries of intramuscular absorption.

The Effect of Mercurial Diuretics on the Alterations in Renal Tubular Function of Salt-Depleted Dogs. LESter Rice, Julian Frieden, Morton Smith and BenJAMIN KaplaN, Chicago, Ill. (Introduced by L. N. Katz).

Salt-depletion provides a convenient method for effecting increased renal tubular sodium reabsorption. Presumably this increased renal tubular capacity for sodium reabsorption results from enhanced distal tubular activity. In an attempt to assess the site of action of mercurial diuretics, a study was made of their influence on renal sodium handling in salt-depleted dogs.

During infusion of $1.5 \%$ sodium chloride solution, saltdepleted dogs exhibited two consistent differences in response to saturation doses of mercuhydrin (2 cc. in- travenously) when compared with these animals in normal sodium balance, viz., (1) the per cent of filtered sodium excreted was lower and (2) the sodium concentration of the reabsorbate was higher than in the controls. Thus mercurialization did not abolish the differences in renal tubular function between dogs in the normal and the salt depleted state. If facultative sodium reabsorption is confined to the distal tubule, these findings suggest that the primary site of action of the mercurial diuretics is the proximal tubule.

Variations in Renal Tubular Reabsorption of Sodium Independent of Change in Adrenocortical Hormone. Jack D. Rosenbaum, Solomon Papper and Milton M. Ashley, Boston, Mass. (Introduced by Henry Jackson, Jr.).

Because adrenocortical hormones exert important influences upon the renal tubular reabsorption of sodium, it has often been suggested that physiologic alterations in sodium excretion may be mediated by changes in endogenous adrenocortical activity. However, in patients with Addison's disease, who lack an endogenous source of adrenocortical hormone, the provision of a constant exogenous supply of desoxycorticosterone acetate and cortisone must establish an essentially uniform hormonal level. In such patients, therefore, large variations in tubular reabsorption of sodium could not reasonably be attributed to changes in the amount of available hormone.

In two patients with Addison's disease, maintained on desoxycorticosterone acetate and cortisone, variations in renal excretion of sodium were studied in relation to (a) change in dietary sodium intake, (b) diurnal excretory rhythm, (c) change in posture, (d) venous congestion of the limbs, (e) hypotonic expansion of extracellular fluid volume (by intravenous infusion of a mixture of sodium chloride and sodium bicarbonate). Under these conditions both patients manifested large changes in excretion of sodium indistinguishable from those observed in normal subjects and independent of alterations in filtered sodium load or endogenous creatinine clearance. These observations indicate that appropriate renal tubular responses to stimuli for sodium conservation or excretion do not require a change in the level of adrenocortical activity.

Hypotonic expansion of extracellular fluid volume was carried out in one of the patients while he was maintained on desoxycorticosterone acetate alone, prior to the administration of cortisone. His failure to manifest a natriuretic response under these circumstances suggests that, although such a response does not require a change in adrenocortical activity, an adequate supply of cortisone-like steroid is required to maintain the integrity of the mechanism involved.

\section{Behavior of Lipids during Electrophoresis of Serum on} Paper. I. N. Rosenberg, Boston, Massachusetts. (Introduced by Samuel Proger).

The association of serum lipids with proteins was studied by performing electrophoresis of human serum on filter paper and comparing the resulting protein and lipid pat- 
terns. Serum ( 0.1 cc.) was applied as a narrow band across a $40 \times 11 \mathrm{~cm}$. sheet of Whatman $3 \mathrm{MM}$ paper, which was then saturated with barbital buffer $(.025 \mathrm{M}$, pH 8.6) and a potential of 320 volts (4.4 milliamperes) was maintained between the ends of the paper for four hours. Longitudinal parallel strips of the dried paper were then stained separately. Proteins were demonstrated by brom phenol blue (Durrum). Fat was stained by Sudan IV (Rossman) and cholesterol either by spraying with antimony pentachloride or by the Schultz reagent (1:1 acetic acid-sulfuric acid) applied to paper pretreated with ferric chloride.

The microelectrophoretic protein patterns indicated four bands tentatively identified as albumin, alpha-, beta-, and gamma-globulin. Sudan stains showed that most of the lipid corresponded closely with the beta-globulin band, much smaller amounts being present in the albumin fraction, and virtually none in the gamma- and alpha-globulins. In some instances a light trail of sudanophilic material extended from the origin to the beta-globulin position, where it was more concentrated. The Schultz and Sudan patterns were similar, most of the cholesterol being in the beta-globulin region.

Intravenous administration of heparin to fasting subjects and to those with alimentary hyperlipemia induced a rapid and marked but transient change in lipid pattern. This was characterized by an increased extent of both sudanophilic and Schultz-reacting zones toward the anode and beyond the beta-globulin band as compared with the pre-heparin pattern. No change occurred in the serum protein pattern.

\section{Comparative Metabolic Effects of the Intramuscular Use of Cortisone Acetate and 17-Hydroxycorticosterone. (Compound F) Acetate. Robert M. Salassa, Marschelle $H$. Power, Harold L. Mason and Randall G. Sprague,* Rochester, Minnesota.}

A comparison was made of the metabolic activity of cortisone acetate and 17-hydroxycorticosterone acetate, administered intramuscularly for prolonged periods, in 3 patients with rheumatoid arthritis. Two of the patients received $3.6 \mathrm{gm}$. of cortisone acetate in twenty-four days on one occasion, and $3.6 \mathrm{gm}$. of 17-hydroxycorticosterone acetate in twenty-four days on another. The third patient received $4.2 \mathrm{gm}$. of cortisone acetate in thirty days on two different occasions, and $4.2 \mathrm{gm}$. of 17-hydroxycorticosterone acetate in thirty days on another. Nitrogen balance and urinary excretion of 17-ketosteroids and corticosteroids were determined.

Both steroids caused an increase in urinary nitrogen. The effect of 17-hydroxycorticosterone acetate on nitrogen balance was slower in onset and less intense than that of cortisone acetate. The decrease in urinary nitrogen after discontinuance of steroid therapy was slower in the case of 17-hydroxycorticosterone acetate. The total effect of 17-hydroxycorticosterone acetate on nitrogen balance was less than that of cortisone acetate.

Evidence that the foregoing differences in metabolic activity of the two steroids were related at least in part to different rates of absorption from the sites of injection was obtained from studies of the urinary corticosteroids and 17-ketosteroids. The increase in urinary corticosteroids after administration of 17-hydroxycorticosterone acetate was slower and less pronounced than it was after administration of cortisone acetate. Urinary corticosteroids decreased more rapidly after discontinuance of administration of cortisone acetate than after discontinuance of the administration of 17-hydroxycorticosterone acetate. The urinary 17 -ketosteroids increased after administration of cortisone acetate, but this effect was less marked or absent after administration of 17-hydroxycorticosterone acetate.

It is concluded that the observed differences in metabolic effects of the two steroids under the conditions of this study are quantitative rather than qualitative, and probably are related at least in part to slower absorption of 17-hydroxycorticosterone acetate from the sites of injection.

Favorable Effects of L-Norepinephrine on Experimental Localized Ischemia of the Myocardium. JоHN J. Sayen, Warner F. Sheldon, Peter T. Kuo, Harry F. Zinsser, Orville Horwitz and Ali F. Sumen, Philadelphia, Penna. (Introduced by Charles C. Wolferth).

In areas of myocardial ischemia produced by coronary obstruction, small doses of 1-norepinephrine cause striking rises in oxygen tension. This has been demonstrated by polarographic estimations of intramyocardial oxygen tension made at multiple areas in the exposed left ventricles of anesthetized dogs.

Coronary occlusion or severe narrowing is followed by a progressive fall in local oxygen tension, failure of muscle contraction and later characteristic changes in the epicardial electrocardiogram. Intravenous injection of 0.5 microgram per kilogram of 1-norepinephrine reverses these evidences of ischemia, while producing marked bradycardia and a slight increase of mean blood pressure but no ventricular ectopic beats. The myocardium outside the ischemic zone shows unchanged or slightly decreased oxygen tension. With single doses favorable changes are transient, but can be reproduced by repetition of the dose or maintained by a slow infusion during prolonged coronary obstruction. After Regitine (C-7337), which partially abolishes both the bradycardia and the pressor effect of 1-norepinephrine, clear-cut beneficial effects in ischemic areas fail to occur.

L-norepinephrine produces greater and more rapid favorable changes in ischemic areas than pure oxygen inhalation. However, oxygen increases the improvement when administered simultaneously. Both agents in combination may produce rises of oxygen tension in large ischemic areas which have failed to show favorable responses to either agent alone.

In contrast to 1-norepinephrine, 1-epinephrine in small doses causes ectopic beats and does not produce definite improvement in ischemic areas. Both drugs in larger 
dosage produce severe hypertension and frequent ectopic beats. While such doses increase the oxygen tension of myocardium which has a normal blood supply, they not only fail to reverse, but sometimes accentuate, the evidences of ischemia in muscle supplied by an obstructed coronary branch.

\section{The Changes in Cerebral Vascular Tone in Experimental} Metabolic Alkalosis and Acidosis. JAMes F. ScHIEve and William P. Wilson, Durham, North Carolina. (Introduced by J. M. Ruffin).

The increased cerebral blood flow (CBF) of respiratory acidosis produced by inhalation of carbon dioxide is associated with decreased $\mathrm{pH}$ and increased carbon dioxide content of arterial blood. The decreased CBF of respiratory alkalosis following hyperventilation is associated with increased $\mathrm{pH}$ and decreased carbon dioxide. It is stated that cerebral vascular tone is predominantly under chemical control, and that, in absence of anoxia, this tone is regulated by either $\mathrm{pH}$ or carbon dioxide content of arterial blood. Previous studies fail to separate which of these two factors is of prime importance.

Metabolic alkalosis and acidosis were established by intravenous infusion of $\mathrm{NaHCO}_{3}$ and $\mathrm{NH}_{4} \mathrm{Cl}$, respectively. CBF (nitrous oxide technique) was measured before and during infusions. $\mathrm{NH}_{4} \mathrm{Cl}$ caused mild hyperpnea and, if infused too rapidly, nausea. No other symptoms were noted. Infusions of isotonic and hypertonic $\mathrm{NaCl}$ served as controls.

Infusions of isotonic $\mathrm{NaCl}$ caused no change in $\mathrm{CBF}$ or metabolism. Hypertonic saline caused a $10 \%$ increase, which was of doubtful statistical significance. Three per cent $\mathrm{NaHCO}_{3}$ produced a $70 \%$ increase in $\mathrm{CBF}$ without changing cerebral oxygen consumption. This increase is comparable to that found while breathing $5 \% \mathrm{CO}_{2}$, but is without the distressing side effects of hyperpnea or hypertension. When these alkalotic subjects breathed $5 \% \mathrm{CO}_{2}$, there was a further increase in CBF while $\mathrm{pH}$ shifted back toward control levels. $\mathrm{NH}_{4} \mathrm{CI}$ caused a $20 \%$ decrease in $\mathrm{CBF}$.

Metabolic alkalosis $\left(\mathrm{NaHCO}_{3}\right)$ increases $\mathrm{CBF}$, and is accompanied by increased $\mathrm{pH}$ and $\mathrm{CO}$. content, while metabolic acidosis $\left(\mathrm{NH}_{4} \mathrm{Cl}\right)$ decreases $\mathrm{CBF}$ and is accompanied by decreased $\mathrm{pH}$ and $\mathrm{CO}_{2}$ content of arterial blood. The CBF changes are reversed from those reported in respiratory alkalosis and acidosis, and are related to changes in $\mathrm{CO}_{2}$ rather than $\mathrm{pH}$. This study suggests that $\mathrm{CO}$, is more important than $\mathrm{pH}$ in regulating cerebral vascular tone.

Experimental Porphyria in Rabbits. RUDI SCHMID and Samuer Schwartz, Minneapolis, Minnesota. (Introduced by C. J. Watson).

We have recently observed that in congenital or photosensitive porphyria, the abnormal porphyrin formation originates in the normoblastic nuclei of the bone marrow (porphyria erythropoietica), whereas in the intermittent acute and the "mixed" types the porphyrins originate in the liver (porphyria hepatica). A condition similar in many respects to the human erythropoietic porphyria, with high concentration of uroporphyrin $I$ in the bone marrow, circulating erythrocytes and urine can be achieved by treating rabbits with a combination of phenylhydrazine, intravenous lead acetate and ultraviolet light.

It has now been found that a condition with the essential features of human hepatic porphyria can be produced in rabbits by oral administration of allylisopropylacetylcarbamid (Sedormid). Such rabbits excrete urinary uroporphyrin and porphobilinogen even in excess of the amounts found in the human disease. The uroporphyrin is of the Waldenström type (methyl ester M.P. $252^{\circ} \mathrm{C}$ ), on decarboxylation yielding approximately equal amounts of coproporphyrin I and coproporphyrin III. In further similarity to the human disease the increased urinary coproporphyrin is principally isomer type III and, like the uroporphyrin, it is excreted as the zinc complex. The bile contains enormous concentrations of copro- and protoporphyrin and has intense red fluorescence. The liver exhibits intense red fluorescence and contains high concentrations of uro-, copro- and protoporphyrin as well as porphobilinogen, whereas the bone marrow and the erythrocyte porphyrin concentrations are within the normal limits.

These findings have obvious implications with respect to human porphyria and porphyrin metabolism in general. They demonstrate for the first time that an hepatic as well as an erythropoietic porphyria can be produced experimentally in the rabbit. They further indicate the likelihood that the liver is able to produce porphyrins independently of the porphyrin formation related to hemoglobin synthesis in the bone marrow.

Comparative Studies on the Concentration of $\beta$-Alanyl Dipeptides in Skeletal Muscle and Heart. GerHard SchMIdT and Ricardo CubiLes, Boston, Massachusetts. (Introduced by Raymond D. Adams).

The $\beta$-alanyl peptides, $\beta$-alanyl-histine (carnosine) and $\beta$-alanyl-1,5-methyl histidine (anserine) are normal constituents of the skeletal muscle of all higher animals. It was of interest to compare the concentration of these $\beta$-alanyl peptides in skeletal muscle and heart. The available methods permit quantitative determinations of carnosine but not of anserine. A chromatographic procedure for the determination of the total $\beta$-alanyl-peptides and of the free $\beta$-alanine was developed in this laboratory. Protein-free tissue extracts were divided into two aliquots, one (A) serving for the determination of free $\beta$-alanine, the second (B) for that of bound $\beta$-alanine. $B$ was refluxed for 2 hours in $2 \mathrm{~N}$-sulfuric acid. After removal of the sulfuric acid with an anion exchanger, the combined filtrate and washings were concentrated under reduced pressure to a small volume. Ascending chromatograms of aliquots of A and B were obtained with a mixture of 75 volumes of butanol and 25 volumes of 50 per cent acetic acid and developed by spraying with a 0.5 per cent solution of ninhydrin in butanol. The blue spots corresponding to $\beta$-alanine were extracted with a 50 per cent aqueous solution of acetone, and the 
extinction of the acetone solutions was determined colorimetrically.

Although the concentration of bound $\beta$-alanine in rat skeletal muscle averaged between 100 and $140 \mathrm{mg}$ per $100 \mathrm{gm}$ of moist tissue, no appreciable amounts of bound $\beta$-alanine were found in hearts of rat, rabbit, and beef.

Only very small, if any, amounts of free $\beta$-alanine were found in skeletal muscle. No free $\beta$-alanine was found in heart tissue.

The Use of the Aromatic Diamidines for Therapy of Systemic Disease Caused by Pathogenic Fungi. Emanuel B. Schoenbach * and Joseph M. Miller, New York City, New York.

Low concentrations of the aromatic diamidines have been shown to inhibit the growth of various species of fungi in vitro. Stilbamidine $\left(4,4^{\prime}\right.$ stilbenedicarboxamidine), which has been used for the treatment of human trypanosomiasis and leishmaniasis by parenteral administration, has been administered to four patients with systemic blastomycosis, one patient with actinomycosis and two patients with torula meningitis.

No evident response was noted in the torula infections but prompt clearing and sterilization of lesions, associated with symptomatic improvement, were observed in the patients with blastomycosis and actinomycosis. A dosage of $150 \mathrm{mg}$ per day given in three courses to a total of 4.5 to 6 grams appears to be sufficient. In some cases local application of propamidine [ $p, p^{\prime}$ (trimethylenedioxy) dibenzamidine] in 0.1 per cent solution, streptodornase and streptokinase have been employed to supplement the parenteral therapy. The only toxic reaction noted has been the development of a late stilbamidine neuropathy.

Further investigation of the aromatic diamidines for the therapy of systemic involvement with pathogenic fungi is warranted.

Mechanism for Control of Hypertension. HENRY A. Schroeder,* H. Mitchell Perry, JR and John D. Morrow, St. Louis, Missouri.

Hydrazinophthalazine can act as a carbonyl reagent and probably inactivates pherentasin, the pressor principle found in hypertensive blood, through combination of hydrazine and ketone groups. This drug is relatively non-toxic and produces a moderate lowering of blood pressure most marked in patients who have had unsuccessful sympathectomy. When autonomic ganglia are blocked by hexamethonium halide an additional factor in the production of hypertension appears to be neutralized. While neither drug alone gives extended control of hypertension at reasonable levels, both together are usually effective in this regard. Of more than 60 patients with severe hypertension of all types, followed for 3 to 7 months, continuous combined therapy controlled all at reasonable levels and half at normotensive levels. In all except one of 30 cases of malignant hypertension the malignant stage reversed itself, but only one-third attained normotensive levels. Apparently cholinergic fibers must be blocked by the regimen to be effective since hexa- methonium halide cannot be replaced by dibenamine or dihydroergot preparations.

If hydrazinophthalazine or hexamethonium halide is given orally for the control of human hypertension, urinary excretion of the unchanged compound begins quickly, and reaches a maximum in several hours; however, total recovery is no more than 5 or 10 per cent of either. After parenteral administration, half or more of the hexamethonium halide is excreted in the urine, whereas there is no increase in the hydrazinophthalazine recovered. The hydrazine moiety of the latter combines in vitro with sulfhydryl or carbonyl groups of several physiologic substances including cysteine, glutathione, and pyruvic acid. That sulfhydryl binding actually occurs in vivo is demonstrable by breaking this linkage in urine of patients and thereby more than doubling hydrazinophthalazine recovery.

Balance Studies in Two Remarkable Instances of "Pure" Chronic Potassium Depletion Resulting from the Overuse of Laxatives. WILliam B. SchWARTZ and ARNold S. Relman, Boston, Massachusetts. (Introduced by Joseph H. Pratt).

Prolonged balance studies were conducted on two young women who had gradually developed severe potassium depletion as a result of chronic diarrhea induced by overuse of laxatives.

Despite the loss of approximately one-third of total normal body potassium content (as estimated by correlation of potassium retention with $\mathrm{K}^{\mathbf{2}}$ dilution data), neither of the patients had overt symptoms or neuromuscular signs. It was only through the discovery of $\mathrm{S}-\mathrm{T}$ and $\mathrm{T}$ wave changes in routine electrocardiograms that hypokalemia was suspected. One patient had a slight increase in serum bicarbonate, and neither had any other disturbance of serum electrolytes except for extreme hypokalemia.

When laxatives were withdrawn, both patients, while on a normal oral intake of potassium and a low normal intake of phosphorus, retained $12 \mathrm{mEq} / \mathrm{kgm}$. of potassium in excess of nitrogen over a period of two weeks, and showed no significant change in the corrected balance of phosphorus. The increase in total exchangeable potassium measured by $\mathrm{K}^{\mathbf{2}}$ closely predicted the actual potassium retention.

In neither case was there any appreciable change in body weight, nitrogen balance, sodium balance, or extracellular tonicity at the end of the period of potassium retention, suggesting that potassium repletion was associated with a reduction in the osmotic activity of some intracellular constituent. Calculations based on the chloride space indicated a large increase in the intracellular concentration of potassium and total cation and a shift of sodium from cells to the chloride space of a magnitude nearly equal to the cellular uptake of potassium.

After potassium retention was complete, when serum potassium and the electrocardiogram had returned to normal, readministration of laxative to one subject praduced diarrhea and a negative potassium balance. The electrocardiogram again became abnormal. This change occurred prior to the fall in serum potassium. 
Blood Ketoglutarate and Pyruvate in Liver Disease. David Seligson, Gerald J. McCormick and Victor Sborov, Washington, D. C. (Introduced by Francis C. Wood).

The specific measurement of alpha-ketoglutaric acid (KG) and pyruvic acid (P) in blood is made possible by a new method using paper chromatography to separate their dinitrophenylhydrazones from other ketones normally present. Because of the known importances of $\mathrm{KG}$ and $\mathrm{P}$ in intermediary metabolism these substances were measured in liver and other diseases to establish a possible relationship between these measurements and hepatic disease.

Eighteen normal males showed a mean KG blood level of $13.0 \pm 1.5$ micromoles/liter. The mean level of $P$ in these individuals was $73 \pm 16$ micromoles/liter. Values above two standard deviations were considered abnormal ( $K G>16.0$ and $P>105$ ). Sixteen of eighteen cases of acute viral hepatitis showed abnormal $K G$ values whereas only five showed elevated $P$ values. The means were $24.0 \pm 11.7$ and $91 \pm 26$ respectively. Of sixteen patients with chronic viral hepatitis thirteen had abnormal $\mathrm{KG}$ and five had abnormal $P$ levels. Of nineteen cases with cirrhosis sixteen had abnormal KG and eleven abnormal $P$ levels.

A series of miscellaneous diseases involving the liver showed abnormally high KG which paralleled other evidences of hepatic dysfunction. Jaundiced patients without liver damage showed normal values.

Serial determinations of $K G$ and $P$ were made in acute viral hepatitis. Although the peak elevation of $\mathrm{KG}$ occurred later than the peak of bilirubin, KG tended to fall with recovery. Fewer of the PA values were abnormal in this group; their fall, however, paralleled the $\mathrm{KG}$ values with recovery. Wide variations of $K G$ and $P$ occurred in the cirrhotics usually associated with therapy, physical activity or change in severity of the disease.

It is concluded that liver damage is associated with an elevation of blood $\mathrm{KG}$ and to a lesser extent pyruvate. It is believed that in liver disease this reflects a defect in intermediary metabolism.

Barbiturate Tolerance Tests in Patients with Liver Disease. J. T. Sessions, JR. and F. J. Ingelfinger,* Boston, Massachusetts.

Clinical studies and liver function tests made by us in patients with stabilized cirrhosis have not substantiated the belief that these patients are highly susceptible to hypnotic doses of pentobarbital. To obtain information on the handling of barbiturates by cirrhotic patients, an attempt was made to measure pentobarbital blood levels by a spectrophotometric technique $(239-240 \mathrm{~m} \mu)$, but this proved unsuccessful following single parenteral doses of the drug. During and following constant infusions of $100-120 \mathrm{mg}$. of pentobarbital, however, rising and falling blood levels were measured satisfactorily. The tolerance curves so obtained in 10 control subjects and in 13 patients with advanced cirrhosis were similar. It seems that the disappearance from the blood of pentobarbital, or early breakdown products, is not affected by hepatic disease.

Barbiturates may diffuse rapidly out of the blood, especially since they are said not to be bound to serum proteins. To test this possibility, simultaneous measurements of pentobarbital were made on blood and ascitic fluid during a constant barbiturate infusion. The tolerance curves obtained in the two compartments were nearly parallel. The increased fluid volume in patients with ascites and the ready diffusibility of pentobarbital may influence barbiturate tolerance curves in such patients.

Pyruvate metabolism is said to be affected both by barbiturates and by liver disease. We have confirmed the finding that blood pyruvate levels tend to be increased in patients with hepatic disease, but constant infusions of pentobarbital did not change these levels.

\section{Studies on the Biologic Significance of the Protection Afforded Intracellular Brucella. James M. Shaffer and Carrell J. Kucera, Minneapolis, Minn. (Intro- duced by Wesley W. Spink).}

Localization of Brucella intracellularly in infected animals or man constitutes an excellent defense mechanism for the bacteria against therapeutic agents or immune mechanisms. An experimental technique to study this problem utilized exudative leukocytes containing large numbers of intracellular Brucella obtained by the in vivo phagocytosis of Brucella in the peritoneal cavity of adult male white rats. These preparations maintained in vitro in a synthetic tissue culture medium revealed a remarkable protection of intracellular Brucella against therapeutic agents, when used alone or in combination. Viable streptomycinsensitive Brucella have been recovered from leukocytes exposed for 24 hours to 25,000 times the minimal concentration of streptomycin required to kill the organism in a broth culture. None of the therapeutic agents now available for clinical use, whether alone or in combinations, were found to be capable of killing all the intracellular Brucella in 24 hours. Streptomycin combined with aureomycin, terramycin, chloramphenicol, or sulfadiazine was more effective than any single therapeutic agent. This protection of intracellular Brucella has been found to be dependent upon intact, viable leukocytes.

Reduction of Neotetrazolium by leukocytes and Brucella has been investigated as a means of evaluating the metabolic status of the cells and parasite before and after exposure to therapeutic agents. This method can be used to differentiate viable from nonviable leukocytes and heat-killed or phenol-killed Brucella from organisms exposed to high concentrations of the antibiotics.

Phagocytosis of Brucella favors the host by clearing the blood stream of organisms and localizing the infection. Intracellular localization favors the bacteria by maintaining them in a viable state and affording protection against attempts to eradicate the organisms from the tissues. This biologic phenomenon accounts in part for the the chronicity of the disease and relapses that occur. 
The Separation of Specific Serum Proteolytic Inhibitors and the Physiological Significance of Increased Proteolytic Inhibition. N. Raphael Shulman, Bethesda, Maryland. (Introduced by Thomas McP. Brown).

Increased serum antiproteolytic activity occurs in association with various diseases. It has generally been assumed that inhibition of trypsin, chymotrypsin, and plasma (fibrinolysin) is caused by a single substance in serum which occasionally increases in disease. The significance of the increase is not understood for there have been no previous reports of changes in accurately measurable biological phenomena occurring consistently with changes in proteolytic inhibition.

A method of measuring proteolytic activity using a radio-actively tagged substrate was developed to permit highly sensitive measurements in media such as serum. At least two separate anti-proteolytic factors were detected in normal serum by differences in their physical and chemical properties and the specificity of their action on the enzymes tryspin, chymotrypsin, and plasmin. One factor specifically inhibited plasmin (plasmin inhibitor) while the other factor inhibited both trypsin and chymotrypsin (trypsin inhibitor). The inhibitors could be separated by chemical fractionation of serum. Increases of proteolytic inhibition with disease were specific either for plasmin or for trypsin and chymotrypsin.

An analysis was made of the relationship of the level of proteolytic inhibition to the clinical and laboratory data on 117 patients with cancer, various acute diseases, and tuberculosis. It was found that elevated trypsin inhibitor is associated with disease processes that bring about cellular destruction and that there is a close correlation between changes in trypsin inhibitor and changes in sedimentation rate. Intravenous typhoid vaccine was found to produce parallel changes in trypsin inhibitor and fibrinogen concentration in humans. It was concluded that the diagnostic significance of increased trypsin inhibition (and chymotrypsin inhibition) is the same as that of increased fibrinogen concentration (or sedimentation rate).

The question of why trypsin inhibitor and fibrinogen concentration vary together and the circumstances which lead to increases in them will be discussed.

The Effect of Negative Pressure Breathing on Renal Function. H. O. Sieker, O. H. GaUer and J. P. Henry, Dayton, Ohio. (Introduced by R. W. Rundles).

Previous animal studies have suggested that receptor areas in the low pressure side of the cardiovascular system within the thorax appreciate changes in effective blood volume and alter urine flow (Henry and Gauer, 1951; Gauer et al, 1951). Decreasing the blood volume in the thoracic viscera by positive pressure breathing is associated with oliguria in humans (Drury et al, 1947). This study was undertaken to determine in man the effect of negative pressure breathing on renal function.

Following five hour control periods, recumbent normal subjects pressure breathed at $-15 \mathrm{~cm}$. water for $45 \mathrm{~min}$ utes. Urine flow, $\mathrm{pH}$, sodium excretion, and glomerular filtration rate were measured. Blood pressure, pulse rate, and respiration rate were also followed. During negative pressure breathing the urine flow showed an average increase of 340 per cent. The urine $\mathrm{pH}$ during this period was unaltered or inconsistently changed. The urine sodium concentration during negative pressure breathing decreased an average of 73 per cent and the total sodium excreted increased only 17 per cent. In 3 of 7 subjects the total sodium excreted during this period did not increase or it fell below control levels. The glomerular filtration rate increased an average of 42 per cent initially but returned to the control value as negative pressure breathing was continued. The pulse rate, blood pressure, and respiration rate were not significantly altered.

The marked diuresis cannot be attributed to alkalosis because the respiration rate and urine $\mathrm{pH}$ were unaltered or inconsistently changed and there was only a slight increase in the total urine sodium excretion. It is suggested that the increased urine flow which resembles "water diuresis" is the result of engorgement of receptor areas in the thorax. These receptors, it is thought, mediate changes in renal function through the endocrine glands and renal hemodynamics.

The Effect upon the Liver of Inhalation of Carbon Dioxide in High Concentrations. J. L. Sims, O. S. OrTH and LUcies Morris, Madison, Wisconsin. (Introduced by Ovid $O$. Meyer).

The finding that increased concentration of carbon dioxide in gas mixtures used for surgical anesthesia was associated with increased hepatic functional impairment suggested a study of the effect upon the liver of this agent alone.

A mixture of one third carbon dioxide and two thirds oxygen was administered by intratracheal tube for periods of 25 to 30 minutes to each of ten dogs. Anesthesia and analgesia resulted. Blood $\mathrm{pH}$ fell to 6.7-6.8. Needle biopsy of the liver, and tests of bromsulfalein clearance, prothrombin time, icterus index, and prothrombin consumption were performed immediately before and after the administration, and at 24 and 48 hours afterward. The prothrombin consumption showed an irregular variation without definite trend. The results of the other tests and the histologic appearance of the liver remained unaltered.

Five deteriorated schizophrenics in whom other forms of shock therapy had been unavailing were anesthetized for 25 to 30 minutes with a mixture of $25 \%$ to $35 \%$ carbon dioxide and $75 \%$ to $65 \%$ oxygen. Cephalin cholesterol flocculation, thymol turbidity, bromsulfalein clearance and icterus index studies performed before, immediately after, and at $24,48,72$, and 96 hours after the administration showed no change. In two patients prothrombin time increased moderately, and in one slightly. In two it remained unchanged. Bromsulfalein clearance studied during the actual period of anesthetization in two patients was greatly slowed. 
It is concluded that carbon dioxide alone, administered in the manner described, produces no significant hepatic damage.

The Effects of Acute Respiratory Alkalosis on Electrolyte Excretion and Renal Hemodynamics in Man. R. B. Singer, J. K. Clark,* E. S. Barker and J. R. ElkinTON,* Philadelphia, Pa.

Voluntary hyperventilation of $1 / 2$ or 1 hour by 5 normal subjects caused abrupt changes typical of respiratory alkalosis in arterial blood, including average rise in $\mathrm{pH}$ of +0.25 , fall in plasma bicarbonate concentration, and fall in calculated $\mathrm{pCO}$, to less than $20 \mathrm{~mm}$. $\mathrm{Hg}$.

Average urinary responses in 8-10 collection periods per subject were: 1) Variable urine flow, the most consistent change being $>60 \%$ drop during the first period after hyperventilation. This decrease was abolished in one subject by maintaining an osmotic diuresis (mannitol). 2) A characteristic acid-base response with rise in $\mathrm{pH}$ to 7.30 and bicarbonate excretion rate to $192 \mu \mathrm{Eq} . /$ min., and fall in ammonia output. 3) Rise in potassium excretion rate of $+170 \mu \mathrm{Eq}$./min., sustained during hyperventilation, and steady fall afterward reaching in the final period $50 \mu \mathrm{Eq} . / \mathrm{min}$. below the control. 4) Rise in sodium excretion rate of +170 , and in chloride $+110 \mu \mathrm{Eq} . /$ min. in $1 / 2$ hour of hyperventilation, with subsequent abrupt fall below the control levels, regardless of whether the hyperventilation was continued or not. 5) Decrease in glomerular filtration rate (endogenous creatinine) averaging $13 \%$ during and after hyperventilation, with variable changes in effective renal plasma flow (PAH).

Thus, only one-quarter of the urinary acid-base response was due to increase in sodium bircarbonate and decrease in ammonia and titratable acid. Increased potassium bicarbonate excretion played the major role, a finding in agreement with the hypothesis of a relationship between the tubular secretion of hydrogen and potassium. These changes are consistent and correlate well with those in $\mathrm{pH}$ of blood and urine. In contrast, the changes in chloride + equivalent sodium excretion appear to be associated with some factor of early hyperventilation which is not blood $\mathrm{pH}$, urine volume, glomerular filtration, or diurnal variability of electrolyte excretion.

\section{Renal Mechanisms of Uricosuria Produced By Benemid}

(p-(di-n-propylsulfamyl)-benzoic acid) and Salicylate.

Jonas H. Sirota and T. F. Yu, New York, N. Y. (Introduced by Alexander B. Gutman).

The effects of Benemid, a potent uricosuric agent for use in chronic gout, upon the renal clearances of urate, inulin, p-aminohippurate and electrolytes were studied and its mechanism of action investigated in 19 gouty subjects. A single $2 \mathrm{gm}$ dose given orally (19.3 to 29.5 $\mathrm{mgm} / \mathrm{kilo}$ ) uniformly produced a marked increase in Curate without affecting $C_{\text {inu1 in. }}$. The $C_{\text {arato }} / C_{\text {inu11n }}$ ratios rose from a mean of 0.0718 (range 0.0502 to 0.103 ) to peak values averaging 0.337 (range 0.118 to 0.547 ). The elevations were evident within 40 minutes, attained peak values within 4 hours and persisted for 24 hours. CPAB was moderately depressed by Benemid; $\mathrm{C}_{\text {potasutum }}$ and $\mathrm{C}_{\text {phosphate }}$ were uninfluenced and $\mathrm{C}_{\text {sodium }}$ and $\mathrm{C}_{\text {ehloride }}$ were inconsistently increased, particularly in several subjects with very marked uricosuria. Benemid strikingly and apparently quite selectively inhibits tubular reabsorption of urate.

Intravenous sodium salicylate produced little change in $\mathrm{C}_{\text {inu11n. }}$ When plasma salicylate concentrations exceeded $15 \mathrm{mgm} \%, \mathrm{C}_{\text {urate }} / \mathrm{C}_{\text {tnultn }}$ rose markedly; between 5 and $15 \mathrm{mgm} \%$ little change in this ratio was noted; below $5 \mathrm{mgm} \%$ significant depression of $\mathrm{C}_{\text {arato }} / \mathrm{C}_{\text {inulin }}$ was consistently observed. Salicylate infused during Benemid uricosuria produced depression of $\mathrm{C}_{\text {urate }} / \mathrm{C}_{\mathrm{inulta}}$ instead of further augumentation, maximum depressions occurring at plasma salicylate concentrations under 15 mgm \%. Salicylate interferences with the Benemid uricosuric effect may be competitive.

A patient with marked Fanconi type glomerulo-tubular imbalance was found to have strikingly decreased reabsorption of urate, among other evidences of tubular damage.' $\mathrm{C}_{\text {urato }} / \mathrm{C}_{\text {inu11n }}$ approximated unity $(0.984)$ but did not exceed it; i.e., there was no indication either of tubular absorption or tubular secretion of urate. As anticipated under these circumstances, Benemid had no effect on urate clearance or urinary urate excretion.

\section{A Comparison of the Metabolism of Fructose and Glucose} in Hepatic Disease and Diabetes Mellitus. Lloyd H. Smith, JR., Richard H. Ettinger and David Seligson, Washington, D. C. (Introduced by Richard B. Capps).

In order to quantitate rates of fructose utilization in diseases manifesting glucose intolerance, standard intravenous fructose and glucose tolerance tests were performed on normals and patients with hepatitis, cirrhosis, and diabetes. Glucose tolerance was abnormal in 27 per cent of the patients with hepatitis, 50 per cent of the cirrhotics, and all of the diabetics. The fructose tolerance curves appeared grossly similar in all groups. It was found, however, that the disappearance rate of infused fructose had the characteristics of a first order reaction; the rate of disappearance, therefore, could be expressed as time of "half-life." Comparison of disappearance rates so expressed revealed significant group differences. The normal group had a mean "half-life" value of 18.6 minutes, the hepatitis group 20.8 minutes, the cirrhotics 21.8 minutes and the diabetics 23.6 minutes. No correlation in individual patients was noted between rate of fructose disappearance and degree of glucose tolerance.

Serum inorganic phosphorus fell rapidly in each group when fructose was infused and returned toward normal within one hour. Following glucose infusion a more gradual and persistent phosphorus fall occurred. A significant difference in phosphorus fall following glucose was noted in comparing the hepatic patients manifesting glucose intolerance and the mildest diabetics.

Blood levels of lactic, pyruvic and alpha-keto glutaric acids showed only slight increases following glucose, the maximum elevations occurring at 60 minutes. When fructose was given there were more rapid and marked elevations (up to $300 \%$ ) in all groups. 
It is concluded that although the infused fructose disappeared from the blood rapidly, the rate was slightly decreased in patients with hepatocellular disease and diabetes. The lack of correlation with abnormal glucose tolerance, the differences in response of serum inorganic phosphorus, and the characteristic changes of the carbohydrate intermediaries suggest qualitative differences in the metabolic pathways of fructose and glucose.

The Lipid Composition of Ultracentrifugates of Serum from Patients with Acute Hepatitis. J. R. SNAvely, W. H. Goldwater, M. L. RandolPh and W. G. UnGLA:UB, New Orleans, Louisiana. (Introduced by $R$. H. Turner).

Employing a quantity ultracentrifugal technic previously described in detail, the distribution of serum lipids of eight patients with acute hepatitis was determined; three of the patients were restudied in early convalescence.

Fasting serum samples were centrifuged at 48,000 r.p.m. for four hours, the column of centrifugate sampled in the liquid state at horizontal levels, and analyses effected for free and total cholesterol, phospholipids, and total fatty acids.

Characteristic changes from the normal pattern were encountered: Deep in the column where phospholipids and cholesterol esters occurred without free cholesterol; both of these lipids were decreased in concentration. Higher, in the medium density zone, where certain low density aggregates composed of cholesterol, phospholipid, and neutral fat accumulate, there was a marked increase in the concentration of all lipids, and this increase was characterized by a relative decrease in cholesterol ester concentration, and a relative increase in neutral fat as compared to that of free cholesterol. In the three patients whose studies were repeated in convalescence, reversion toward the normal pattern was observed.

As we have indicated previously, acute hepatitis is associated with the appearance of increased concentrations of low density lipoproteins. We suggest that this increase in light aggregates rich in neutral fat and poor in cholesterol ester is a manifestation of increased mobilization of depot fat with sluggish removal by the diseased liver, and that the decrease in phospholipids and cholesterol esters not associated with free cholesterol is a manifestation of defective hepatic synthesis.

Response to Psychological Stress in Persons who are Potentially Hypertensive. MaURICE SokoLow,* R. E. Harris, L. G. Carpenter, M. Freedman and S. P. Hunt, San Francisco, Calif.

Investigations of patients with essential hypertension have led us to recognize certain personality and behavior patterns characteristic of the disease. Their relevance for etiology, however, remains obscure. A demonstration that abnormal personality reactions, particularly in situations involving stress, exist before the onset of fixed hypertension would add plausibility to the argument that personality factors play a role in the causal chain of events leading to hypertension. To this end, 39 college women who had shown elevations of blood pressure on routine initial physical examinations were matched with 39 controls wtih low normal pressures. It has been shown that more of the former group will develop hypertension in later life, and hence may be assumed to be "prehypertensive."

Controlled experimental stress was induced by staging inter-personal situations in which the subjects played assigned roles. One psychodrama involved simple frustration; the second, criticism, rejection, and hostility from an authoritative person. Psychological tests were obtained before and after the psychodramas. Behavior during the experimental situations was observed and recorded. A psychiatrist interviewed the students, attempted to identify their characteristic responses to stress and to infer which were pre-hypertensive. The testinterpreters, observers, and psychiatrist did not know whether the subjects were pre-hypertensive or control until the data had been analyzed.

Most of the procedures showed clearly significant differences between the groups. The pre-hypertensives were typically more hostile and less well controlled than the normals and, in general, less well-equipped to cope with experimental stress.

Although the etiology of essential hypertension is complex, probably involving constitutional and other factors, our data show that the individuals who reacted less adequately to experimentally induced stress were those who demonstrated an elevated blood pressure on the initial college examination and, therefore, may be presumed to react similarly to other situations of stress and ultimately to develop fixed hypertension.

\section{Studies of Mineral Metabolism in Disturbance of In-} testinal Absorption. Herta Spencer and IsAac Lewin, New York City. (Introduced by B. S. Oppenheimer).

The mechanism of apparent faulty intestinal absorption and its effect upon mineral metabolism and electrolyte balance was studied under metabolic conditions in three cases. One patient had a resection of the major part of the small intestine for sarcoma; two patients had ideopathic steatorrhea associated with hypocalcemia and tetany. On a low calcium diet the derangement of mineral metabolism consisted of an abnormally high fecal and an abnormally low urinary calcium excretion, leading to a markedly negative calcium balance. However, this balance became markedly positive by simply increasing the calcium intake. This suggests that intestinal calcium absorption and utilization are not at fault. The great need for calcium was demonstrated also, by the intravenous calcium tolerance test which showed an extremely low 24 hour calcium excretion indicative of the skeletons avidity for this mineral.

Balance studies of sodium and potassium have shown that considerable quantities of electrolytes were lost through the intestinal canal and that these losses could be well correlated with the high fluid content of the 
feces. The total electrolyte concentration of the fecal fluids approximated that of a "plasma-ultra-filtrate." This indicates that the primary defect is the loss of fluids with concomitant loss of electrolytes rather than defective intestinal absorption.

Metabolic studies have also shown that the body is able to compensate partially for losses of electrolytes through the intestinal canal by decreased renal excretion of these substances. Chronic metabolic studies and the application of the intravenous calcium test were helpful in elucidating the mechanism of intestinal disturbances. The effect of ACTH on the clinical course and on metabolic changes will be discussed.

\section{Immunologic Aspects of Idiopathic Thrombocytopenic} Purpura (ITP). Mario Stefanini, Jyoti B. Chatterjea and Edward Adelson, Boston, Massachusetts. (Introduced by William Dameshek).

Recent evidence indicates that the thrombocytopenia of ITP may be immunologic in origin. Antiplatelet substances were accordingly searched for in the sera of twenty patients with ITP. Sera were tested against platelets for agglutination, precipitation, lysis, complement fixation, inhibition of physiologic properties of platelets. Various precautions in handling sera and platelets were taken to prevent non-specific platelet agglutination.

Low titer $(1: 8)$ platelet agglutinins were found in two patients. A third patient exhibited a high titer agglutinin $(1: 1280)$ causing agglutination of human but not animal platelets. The agglutinin had continued activity after submitting the serum to: (a) acidification ( $\mathrm{pH} 5)$ or alkalinization ( $\mathrm{pH} \mathrm{9)} \mathrm{;} \mathrm{(b)} \mathrm{temperatures} \mathrm{rang-}$ ing from $4^{\circ}$ to $66^{\circ} \mathrm{C}$.; (c) absorption with gels; (d) filtration through Seitz and dialysis; (e) absorption with human erythrocytes and leucocytes, beef erythrocytes, guinea pig kidney; (f) storage at $4^{\circ} \mathrm{C}$. The agglutinin was present in the gamma globulin fraction of serum.

When injected in humans the patient's plasma induced: (a) severe, sustained thromobocytopenia, with circulating large and bizarre platelets; (b) increased number and size of megakaryocytes with vacuolization and lack of platelet production; (c) demonstrable platelet agglutinin in recipient's serum for 12-14 days.

Seven of eleven patients showed significant Forssmanlike antibody against sheep erythrocytes. In one, heteroplatelet cold agglutinin was detected.

In all patients with ITP disappearance of injected platelets was prompt. Plasma of several ITP patients produced severe or moderate thrombocytopenic response in normals. Inability to demonstrate antiplatelet agents in vitro more frequently emphasizes: (a) need of finer technics for detection of antiplatelet substances; (b) apparently great variability of pathogenetic mechanisms in ITP. Combined studies of this type already indicate the possibility of discriminaing between different types of ITP and of predicting the therapeutic effects of splenectomy.
Observations on the Storage Lesion of the Erythrocyte. Alexander Stevens, Beverly Gabrio, Richard Czajkowski and Clement Finch,* Seattle, Washington.

Sequential physical and biochemical changes develop in the human erythrocyte when stored in vitro. These changes are retarded by a low storage temperature and by the addition of glucose.

The "storage lesion" is not related to cell senescence as it occurs in vivo. Young cells are even more vulnerable to storage damage than are old cells. The concentration of leucocytes and the degree of oxygenation have significant effects on the rate of development of the "storage lesion." However, in all instances metabolic alterations attributable to storage appear.

Manipulations with plasma constituents in vitro have not appreciably affected the progress of storage changes nor influenced post-transfusion survival. The biochemical characteristics of the "storage lesion" may be rapidly reversed upon reintroduction of the stored erythrocyte into the blood stream. Red cells have been stored for three weeks, transfused, and withdrawn for a second three weeks of storage. The post-transfusion survival after the second period of storage was excellent.

These observations indicate that in the storage of the erythrocyte aging as it occurs in vivo is not a limiting factor. While plasma appears inadequate to support normal red cell metabolism, the lesion produced by storage is rapidly reversible in vivo.

Effect of Steroid on Potassium Exchange in Blood. Hirsh Sulkowitch, Susan Butler and Benedict F. Massell, Boston, Mass. (Introduced by Louis $K$. Diamond).

The mechanism by which steroids influence the transfer of potassium between tissue cells, and their surrounding extracellular fluid is not known. Whole blood was employed as a relatively simple in vitro system for studying this problem.

Cortisone was added to freshly drawn heparinized whole blood and incubated with controls in a roller tube device. During the first hour of incubation, there is a transfer of potassium from cells into plasma. The quantity of potassium transferred in the cortisone-containing blood was many times that exchanged in the controls. After the first hour, in both the cortisone containing blood and the controls, there is a transfer of potassium back into the red cell which is maintained to approximately the fourth hour. The general patterns of the cortisone and control curves are similar, but at all times after the zero hour the concentration of potassium in the plasma is much higher in the cortisone containing samples than the controls.

Of the other steroids studies, only testosterone influenced the transfer of potassium. 
Physiological Behavior of Actomyosin Strips of Heart Muscle. Max TAeschler and R. J. Bing,* Birmingham, Ala.

Strips of heart muscle of $2 \mathrm{~mm}$. diameter and $3-4 \mathrm{~cm}$. in length were dissected from the ventricular muscle of dogs. The muscle was washed for 8 days in $50 \%$ glycerol according to Szent-Gyorgyi. From these preparations, muscle fibers of from .4 to $.7 \mathrm{~mm}$. diameter and a length of $2 \mathrm{~cm}$. were dissected. The muscle was suspended in an isotonic solution with a cation composition identical to that of heart muscle. Isotonic and isometric contractions were recorded following the addition of $.8 \%$ of the $\mathrm{Na}$ salt of Adenosinetriphosphate (A.T.P.). The work was calculated as the product of contraction times load. Heart muscle preparations behaved differently from skeletal muscle (rabbit psoas), since in the former the tension developed and the speed of contraction were less. When the preparation was loaded and its contraction recorded isotonically, the degree of contraction and work performed were proportional to the logarithm of time. Increasing the initial length by augmenting the load resulted in a greater output of work. However, the speed and amplitude of contraction were decreased. After a certain load (about $120 \mathrm{gms} . / \mathrm{cm}^{2}$ fiber area) was exceeded, the work declined. When the muscle strips were stretched to $20 \%$ of their original length during extraction, the work output was significantly increased. However, the relationship of load to work remained qualitatively unchanged. Excessive stretching during extraction reduced the work to values observed on the unstretched muscle. The results indicate that many physiological features of the intact heart are inherent properties of the contractile proteins of heart muscle.

The Hemorrhagic Syndrome of Metastatic Prostatic Cancer and its Treatment. Henry J. TAgnon,* PhyLlis Schulman, Willet F. Whitmore and Sheldon C. Kravitz, New York, N. Y.

Bleeding manifestations are a frequent complication of metastatic cancer of the prostate. In many instances, this hemorrhagic tendency is not associated with thrombocytopenia. We have observed that the hemorrhagic syndrome of metastatic prostatic cancer is caused by the presence of a proteolytic enzyme in the blood of the patients. This enzyme proteolyzes fibrinogen and fibrin as well as one or several of the factors entering into the first phase of the coagulation reaction, thereby prolonging the one-stage prothrombin time. The proteolysis of fibrin appears to be the main cause of the deficient hemostasis. The enzyme is present in normal as well as in the cancerous prostatic tissue and we were able to extract also from the metastases. This fibrinolysis constitutes a new example of the release into the blood of an enzyme in metastatic carcinoma of the prostate. The analogy with acid phosphatase is obvious.

This new pathogenesis of the hemorrhagic syndrome of metastatic prostatic cancer led us to the development of a method of treatment based on the use of a specific inhibitor. This could be injected intravenously, was non- toxic, and completely abolished the action of the enzyme in vivo as well as in vitro, thereby providing an effective treatment of the hemorrhagic tendency. This treatment proved also valuable by making possible surgical treatment in cases with hemorrhagic tendencies.

It is proposed to call this newly recognized syndrome "prostatic fibrinolysis."

Inhibition of Influenza Virus Multiplication by Benzimidazole Derivatives. IGor TAMM, KarL FolKers and Frank L. Horsfall, JR.,* New York, N. Y.

Because certain analogs of vitamins are known to affect the metabolic processes of living cells in definite ways, it seemed possible that some might also cause inhibition of influenza virus multiplication. Clear evidence of such inhibition has been obtained with a number of synthetic vitamin analogs. The most interesting substance yet employed is 2,5-dimethylbenzimidazole, an analog of 5,6dimethylbenzimidazole which is a moiety of vitamin $B_{18}$. In a concentration of $0.002 \mathrm{M}$, this compound restricted the multiplication of influenza A (PR8) or B (Lee) virus to about 10 per cent of the control value and did not affect the rate of oxygen consumption of the host tissue.

Experiments were carried out under strictly controlled conditions in vitro: Portions of normal chorioallantoic membrane in chemically defined media were incubated in Warburg vessels or in test tubes which were shaken mechanically. After inoculation, maximal viral concentrations, as measured by hemagglutination, were attained in 30 to 40 hours. 2,5-Dimethylbenzimidazole had no inactivating effect upon the infectivity or the hemagglutinating capacity of influenza viruses. Inhibition of multiplication was obtained when the substance was introduced before or after the virus. Attempts to block the inhibition with vitamin $B_{12}$ or riboflavin have failed.

The available evidence suggests that 2,5-dimethylbenzimidazole interrupts the multiplication of influenza viruses through host cell metabolic mechanisms which are not directly related to respiration. The structure of the compound raises the possibility that it may affect nucleic acid metabolism.

\section{Chloride "Space" and "Mass" Measured with Long-Life} Radiochloride, $\mathrm{Cl}^{\mathrm{s}}$, in Intact Man. S. A. Threefoot, G. E. BURCH * and C. T. RAY, New Orleans, Louisiana.

Long-life radiochloride, $\mathrm{Cl}^{\infty}$, has made possible the determination of chloride space after equilibrium of distribution has been reached (24-120 hrs.).

Chloride space and chloride mass were determined daily for $30-60$ consecutive days by the isotope dilution method and from curves of biologic decay rates in intact man (two control subjects, two with moderate congestive heart failure and two with severe failure).

Chloride space in serum equivalents was larger in those subjects with failure, especially with edema. The overall average space was 22 and $32 \%$ of body weight for the controls, 40 and $50 \%$ for those with moderate, and about $60 \%$ for those with severe failure. Calculated chloride 
mass for the control subjects was 40.5 and $58.9 \mathrm{Gm}$. (0.79 and $1.24 \mathrm{Gm} . / \mathrm{kg}$. body wt.), the patients with moderately severe congestive failure 71.6 and $85.2 \mathrm{Gm}$. (1.33 and 1.83 $\mathrm{Gm} . / \mathrm{kg}$. body wt.) and the patients with severe failure 88.7 and $147 \mathrm{Gm}$. (2.02 and $2.21 \mathrm{Gm}$./kg. body wt.). Wide daily variations in both the calculated chloride space and calculated chloride mass were especially noticeable in the subjects with failure.

In explanation of the fluctuating nature of calculated chloride space and mass, especially without concordant variations in body weight, it is postulated that the chloride compartments of the body not only are anatomically and functually subdivided but also present timeto-time variations in rate of chloride exchange with the serum compartment. Apparently there is "blinking" in activity of the subcompartments of exchangeable chloride. This "blinking" is influenced by several factors, for example, variations in the state of congestive heart failure, spontaneous and therapeutically induced, and dietary intake of chloride. Data obtained conform with the postulates. These phenomena are complex and readily lead to error in calculation of chloride "space" and "mass."

\section{Changes in the Protein-Bound Iodine and the Clinical Course of Thyrotoxic Crisis Treated with ACTH.} Beverly T. TOWERY, Nashville, Tennessee. (Introduced by J. Allen Kennedy).

Certain observations suggest that the initiation of thyrotoxic crisis or its severity may be contingent upon an inadequate adrenocortical response to the stress imposed by severe thyrotoxicosis. Further evidence in support of this hypothesis was obtained during the course of an unequivocal thyroid crisis treated with ACTH alone.

The patient was a 32 year old male with uncomplicated thyrotoxicosis. The events which antedated the crisis were: Graves' disease for 10 months; temporary remission under iodide therapy; no therapy for 6 months; recrudescence of thyrotoxicosis; resumption of Lugol's therapy two weeks prior to hospitalization. Upon admission this medication was discontinued and radioiodine therapy was given on the 14th hospital day.

The first evidence of impending crisis appeared on the 17 th hospital day with the onset of nausea; shortly thereafter the temperature rose to $101.6^{\circ} \mathrm{C}$. and manifestations of thyrotoxic crisis were alarmingly evident.

The intravenous administration of ACTH $(10 \mathrm{mg}$. per liter of dextrose solution) was started immediately and repeated on two successive days. Marked clinical improvement occurred within 18 hours and all manifestations of crisis virtually disappeared within $\mathbf{4 8}$ hours.

Coincident with crisis there was a striking increase in the serum protein-bound iodine concentration and a sharp decrease during the exhibition of ACTH. The circulating eosinophils were relatively high during the crisis and completely disappeared in response to the initial dose of ACTH.

The most plausible interpretation of these observations is provided by the conclusion that the thyroid crisis was associated with a lack of sufficient endogenous corticotropin to evoke an adrenocortical response commensurate with the exigency but that such a response could be achieved with exogenous ACTH. The implications of this hypothesis will be discussed in relation to the rise in PBI in thyroid crisis.

The Site of Synthesis of Creatine in Human Subjects. Frank H. Tyler,* Avery A. Sandberg and Hans H. HeCre,* Salt Lake City, Utah.

It has been demonstrated that kidney tissue is capable of making glycocyamine (GAA) from arginine and glycine and that creatine is normally formed by methylation of GAA in the liver. These observations are based on experiments with mammalian tissue slires and hemogenates. Because of our interest in the creatine anomaly of progressive muscular dystrophy we desired to determine if this mechanism was true in the human.

i Blood samples were obtained from the hepatic and renal veins in six healthy young adult males by vascular catheterization. Simultaneously, arterial samples were obtained, and serum levels of creatine, cl eatinine, GAA, and arginine were determined in etclt sample. The averaged values (expressed as $\mathrm{mgm}$. per $100 \mathrm{ml}$. of serum) obtained in the arterial blood were: GAA 0.23 , creatine 0.35 , creatinine 0.86 , arginine 1.20 . Hepatic venous levels were: GAA 0.19 , creatine 0.84 , creatinine 0.94 , arginine 1.08. The values in the renal venous system were: GAA 0.28 , creatine 0.43 , creatinine 0.73 , arginine 1.34 .

These data relate only to changes in concentration but they demonstrate that GAA formation by the kidney is small when compared to the drop in GAA concentration which occurs across the splanchnic circulation. The slight increase in GAA and creatine concentration of renal venous blood seems to account for only a small proportion of total endogenous creatine formation at estimated rates of renal and splanchnic blood flow. However, the sharp rise in creatine concentration across the splanchnic area as determined from hepatic vein blood is sufficient to account for the known rate of endogenous creatine formation. It would appear that creatine is formed primarily in the splanchnic area, probably by the liver. At least some of the creatine synthesized in that area seems to come from non-glycocyamine precursors.

Studies of Vitamin $B_{12}$ in Human Serum. Walter G. Unglaub and Harold L. Rosenthal, New Orleans, Louisiana. (Introduced by Grace A. Goldsmith).

A method for the assay of vitamin $B_{1 s}$ in serum, employing Lactobacillus leichmannii, has been developed and the serum concentrations of this vitamin have been studied in normal subjects and in patients with macrocytic anemia.

The average serum concentration of vitamin $B_{12}$ in twelve normal subjects in the fasting state was 0.21 millimicrogram per $\mathrm{ml}$. (standard deviation \pm 0.08 millimicrogram). In three normal subjects maintained on 
their usual diets, serum levels obtained at four hour intervals during a 24 hour period ranged from 0.11 to 0.42 millimicrograms per $\mathrm{ml}$. with no constant pattern of variation.

Oral administration of 500 micrograms of vitamin $B_{1 s}$ to two normal subjects and of 1000 micrograms to a third was followed by slight increases in serum vitamin $B_{12}$ which were not considered significant.

In one patient with pernicious anemia serum vitamin $B_{19}$ was 0.07 millimicrograms per $\mathrm{ml}$. during fasting and rose following oral administration of 500 micrograms of vitamin $\mathrm{B}_{12}$ to a maximum of 0.32 millimicrograms per $\mathrm{ml}$. after four hours, declining to 0.19 millimicrograms after 12 hours. A definite hematopoietic response followed this procedure.

Urinary excretion of vitamin $B_{12}$ in the four subjects who received oral doses was not significantly increased.

One patient with macrocytic anemia and extensive neurological changes compatible with subacute combined sclerosis excreted quantities of vitamin $B_{12}$ in the urine which were thirty times that found in normal subjects. In this patient, serum vitamin $B_{12}$ during fasting varied from 0.12 to 0.73 millimicrograms per $\mathrm{ml}$. Following parenteral administration of 100 micrograms and later of 500 micrograms of vitamin $B_{12}$, serum concentrations rose to 3.84 and 24.6 millimicrograms per $\mathrm{ml}$. respectively at the end of one hour, were moderately elevated after 8 hours and slightly above the pre-test level after 24 hours. Implications relative to vitamin $B_{12}$ metabolism will be discussed.

Fate of Intravenously Injected Labelled Gamma Globulin in the Normal Subject and in Patients with Rheumatoid Arthritis. J. H. Vaughan, A. Armato, J. Goldthwaite, P. Brachiman, C. B. Favour and T. B. BAyles, Boston, Massachusetts. (Introduced by Eugene Eppinger).

Separate pools of gamma globulin from normal subjects and patients with rheumatoid arthritis, obtained as Fraction II by Cohn's method of alcohol fractionation, have been labelled with $I^{1 m}$. Each pool has been injected into four normal subjects and four patients with active rheumatoid arthritis. The disappearance of the material from the plasma of the recipients has been traced; the localization of radioactivity among the plasma proteins of the recipients has been defined, and in some instances a quantitative evaluation of the radioactivity appearing in the urine has been made.

In five experiments the average half-life of injected gamma globulins in normal subjects was found to range from 7.0 to 8.8 days. In each instance simultaneously injected rheumatoid arthritis patients evidenced a shorter half-life, the average difference being 1.8 days. There were no data to suggest that the gamma globulins from rheumatoid patients differed structurally from those of normals, in that none of the recipients appeared to differentiate between the two. Fractionation of recipients' plasmas immediately after and at one and two week intervals after injection revealed all radioactivity to be retained within the globulins of Fractions I+ II + III, with no significant amount in Fractions IV $+V$. Urinary radioactivity was not associated with protein and generally was about $75 \%$ of the amount that would be expected to appear from a consideration of the plasma decay curve. Stool analyses were not done.

In three experiments the rate of disappearance of the injected material was significantly accelerated in both normal subjects and rheumatoid arthritis patients, apparently on the basis of partial denaturation incident to the ioslation and labelling procedures.

Scanning of the accessible surface of the body with a beta-gamma survey meter showed no evidence for organ localization of the injected material, neither that from normal nor that from rheumatoid donor pools.

\section{Turn-over Rates of Serum Proteins in Cirrhotic Man.} Wade Volwiler, Kenneth Fremont-Smith and Marion P. MacMartin, Seattle, Washington. (Introduced by Robert S. Evans).

Following oral administration of S-35 tagged dl-cystine, turn-over rates of serum total protein, total globulins, albumin, gamma globulin, and Cohn fraction I + III have been measured in 3 patients with advanced cirrhosis and in 2 normal subjects.

Apparent average values obtained for molecular halflife and per cent of the total fraction in the plasma pool turned over daily were as follows: total protein, cirrhosis 22.4 days or $3.5 \%$, normals 9.6 days or $7.3 \%$; albumin, cirrhosis 20.6 days or $3.4 \%$, normals 17.1 days or $6.0 \%$; total globulins, cirrhosis 11.8 days or $5.9 \%$, normals 7.5 days or $10.7 \%$; gamma globulin, cirrhosis 25.0 days or $3.1 \%$, normals 27.0 days or $2.6 \%$; Cohn fraction $I+I I I$, cirrhosis 13.5 days or $7.7 \%$, normals 4.5 days or $16.2 \%$.

Because of the small number of subjects and the degree of variation observed, the apparent discrepancies between cirrhotics and normals may not be significant. It is believed these apparent half-times are falsely lengthened by isotope reutilization. Attention is called to the very rapid turn-over time in normals of Cohn fraction I + III, which contains about $60 \%$ of the protein-bound lipids.

Inhibition by Cortisone and ACTH of the Fever-Producing and Lymphopenic Action of Influenza Virus. Robert R. Wagner and F. Douglas Lawrason, New Haven, Conn. (Introduced by Edward C. Curnen).

The intravenous injection of influenza virus in rabbits is followed by a characteristic febrile and lymphopenic response. These manifestations of toxic action are not noted when animals are given a second injection of the same virus 24 hours later. The febrile response can be prevented by premedication with antipyrine, but the lymphopenia is unaffected by this drug.

When rabbits were given a single intramuscular injection of cortisone (15 mg.) they developed lymphopenia which was less marked than that produced by influenza virus. Within 12 hours following the administration of cortisone the absolute number of circulating lymphocytes 
returned to pre-injection levels. When tested after this interval, these animals showed partial to complete refractiveness to both the pyrogenic and lymphopenic actions of the PR8 and Lee strains of influenza virus. A similar, but less marked, tolerance to the toxic effect of these viruses was produced when ACTH (12 mg.) was given by the intramuscular or intravenous route 6 to 24 hours prior to virus challenge. Exposure to cold or the injection of lipo-adrenal extract did not induce a similar refractory state. Neither cortisone (15 mg.) nor ACTH (12 mg.) affected the febrile response of rabbits to a minimal pyrogenic dose of typhoid vaccine.

When ACTH ( $6 \mathrm{mg}$.) was given intravenously to rabbits there followed in 2 to 4 hours a reduction in the number of circulating lymphocytes amounting to 60 per cent of the pre-injection mean level. This action of ACTH was not demonstrable in animals which had received a large intravenous dose of the Lee strain of influenza $B$ virus 24 hours previously.

\section{Effects of Oral and Intravenous Gastric Juice upon Vita- $\min B_{13}$ Activity in Pernicious Anemia. RALPH $O$. Wallerstein, John W. Harris and Robert F. Schill- ING, Boston, Mass. (Introduced by George P. Berry).}

Observations were made in patients with pernicious anemia to determine (1) whether there was evidence that parenterally administered gastric juice could render Vita$\min B_{1}$ hematopoietically more active, and (2) whether the potentiating effects of orally administered gastric juice upon Vitamin $B_{1 s}$ involved a stoichiometric relationship between these substances.

A pool of gastric juice (containing no Vitamin $B_{1 s}$ ) was obtained from normal donors, brought to neutrality with $20 \% \mathrm{NaOH}$, rendered sterile by passage through a Berkefeld filter, and stored frozen at $-32^{\circ} \mathrm{C}$.

(1) Five patients given incubated mixtures of $0.33 \mu \mathrm{g}$ Vitamin $B_{1 s}$ and $10 \mathrm{ml}$ inactivated gastric juice daily intravenously made moderate reticulocyte responses. When, in a second period, identical quantities of active gastric juice and of vitamin $B_{12}$ were given, 4 patients made no second reticulocyte peaks; the fifth patient showed a second rise. In a third period, all 5 patients made marked reticulocyte responses to $1 \mu \mathrm{g}$ Vitamin $\mathrm{B}_{18}$ daily intramuscularly.

(2) Two patients were given daily oral mixtures of $1 \mu \mathrm{g}$ Vitamin $B_{1 s}$ and $10 \mathrm{ml}$ gastric juice in an initial period and made moderate reticulocyte responses. Second reticulocyte responses appeared, when, with the same amount of gastric juice, the Vitamin $\mathrm{B}_{18}$ was increased to $5 \mu \mathrm{g}$ daily. Two other patients were given daily oral mixtures of $1 \mu \mathrm{g}$ Vitamin $B_{12}$ and $10 \mathrm{ml}$ gastric juice in an initial period. They made moderate reticulocyte responses when, in a second period, wih the same amount of Vitamin $B_{13}$, the gastric juice was increased to 25 or $50 \mathrm{ml}$ daily.

These findings suggest that because hematopoietic potentiation of Vitamin $B_{12}$ by gastric juice was not shown to take place parenterally, an enteral effect leading merely to enhanced absorption of Vitamin $B_{13}$ is involved upon oral administration, but without a stoichiometric relationship between Vitamin $B_{1}$ and gastric (intrinsic) factor.

\section{Measurement of Extracellular Fluid with Radiosulfate. Mackenzie Walser, Donald W. Seldin * and Arthur Grollman, Dallas, Texas.}

For use in the measurement of ECF, tracer ions have the theoretical advantages of rapid diffusion and freedom from osmotic effects. Most ions studied, including sulfate, have shown progressive cell penetration. Sulfate penetrates cells very slowly, however, although it leaves the plasma as rapidly as sodium. The volume of distribution of radiosulfate after attainment of equilibrium across the capillary membrane was therefore investigated as a measure of ECF.

Following intravenous injection of radiosulfate in normal subjects the plasma concentration decreased at a simple exponential rate after the first 15-20 minutes in man (25-30 in dogs), for as long as 5 hours. The volume of distribution, calculated as the amount injected minus the amount excreted divided by the plasma concentration, increased linearly with time at the same rate in human subjects and dogs. After bilateral nephrectomy in dogs the volume of distribution increased only $6 \%$ between 25 minutes and 5 hours.

Preliminary results of muscle analysis following administration of radiosulfate to nephrectomized dogs indicate that radiosulfate space of muscle remains less than chlorine space of muscle.

It was concluded that equilibrium of distribution across the capillary membrane was virtually complete in about 18 minutes in man and about 27 minutes in dogs. In support of this hypothesis, analysis of the early arterial curves revealed that a single rate $(0.27-0.28$ in man) accurately described the initial disappearance.

Estimates of ECF by this means gave values averaging $17 \%$ of body weight in man and $21 \%$ in dogs. Simultaneous radiosulfate and inulin or mannitol spaces corresponded in most cases, and changed by similar amounts when ECF was altered. Results were reproducible within 0.3 liter. Changes induced by water loading, water deprivation (in diabetes insipidus), and other means equalled simultaneous changes in chloride space.

\section{Demonstration in Man of Type Specific Immunity to Streptococcal Infections. LeWIS W. WanNaMaker and Floyd W. Denny, Cheyenne, Wyoming. (Intro- duced by Charles H. Rammelkamp, Jr.).}

Although animal experiments indicate that immunity to Group A streptococcal infections is type specific, the nature of resistance to such infections has not been well defined in man. In an attempt to clarify the role of type specific immunity in human disease, the spread of Group A streptococci was studied in a group of 43 men housed in a single barrack. Type 14 bacteriostatic antibodies were demonstrated in 13 individuals. Twenty-two men were considered to have no antibodies to this type. The remaining 8 men were carriers of Group A streptococci. 
Five harbored type 14 organisms and one each was a carrier of types 1,5 , and 6 . Bacteriological and clinical observations were made three times a week for a period of six weeks.

In both the group with antibodies and the group without antibodies approximately seventy-five per cent of individuals harbored streptococci in the nose at some time during the observations, and in approximately half of both groups type 14 organisms were found in the nose. Although this suggests that the opportunity for infection was equal in both groups, the incidence of parasitism of the nose and throat, clinical disease, and immunological response was related to the presence of type specific antibodies. Seven of the 22 men without antibodies to type 14 streptococci were infected with this organism but none of the men with demonstrable antibodies were parasitized by this type. In contrast, parasitism with a heterologous type of streptococcus occurred in 6 of the 22 men without antibodies and in 6 of the 13 men with antibodies. Concomitant symptoms and signs of an upper respiratory infection developed in approximately half of the individuals parasitized. These observations suggest that the antibodies measured have a protective action against infection with streptococci of a homologous type but no protective action against infection with streptococci of heterologous types.

The Use of Modified Globin as a Protein Supplement in Normal Individuals. C. Waterhouse, L. D. FenNinger and E. H. Keutmann, Rochester, New York. (Introduced by Howard B. Slavin).

Experimental studies in animals have indicated that the protein moiety of hemoglobin can readily be utilized after erythrocytes have been broken down, and the resultant synthesis of such complete proteins as plasma albumin has been demonstrated.

Modified human globin, with a molecular weight of about 34,000 has been prepared by partial hydrolysis of erythrocytes. ${ }^{1}$ Four patients, receiving a basic diet low in protein but adequate in calories, were given this substance intravenously for 6 to 10 days in amounts of 32 to 64 grams daily, following suitable control periods. Metabolic studies were carried out in a manner similar to that used previously with human serum albumin. The results were not entirely comparable because the desired caloric intake was not maintained in any patient after several doses of globin. Nevertheless, certain fundamental differences between the metabolism of globin and albumin are apparent.

There was no sustained increase in the plasma osmotic pressure, such as is seen when albumin is given. The body weight and hematocrit were constant, and no significant accumulation of globin in the blood occurred even after repeated injections.

Globin was excreted in the urine in considerable quantities each day. Urinary excretion did not increase with repeated administration but depended on the quantity

1 Prepared by Sharp and Dohme, Incorporated. given on any particular day and represented about 25 per cent of that injected.

About 30 per cent of the globin administered appeared within 24 hours in the urine as nitrogen in excess of globin excretion. Good utilization of the remaining globin (as determined from the phosphorus balance corrected for calcium exchange) occurred readily when the caloric intake was adequate.

In contrast to albumin with a half disappearance time of about 5 days, the breakdown of modified globin is rapid. The amino acid components then seem to be handled as might any injected amino acids, subject to the general laws of protein metabolism.

Effect of Arteriovenous Fistula on the Circulation. RENE Wegria,* Charles W. Frank, Hseur H. Wang and Robert Mrluer, New York, N. Y.

Dogs were anesthetized with chloralose and their chest was opened through a midsternal incision. Under artificial respiration, two rotameters were inserted into the arterial side of the systemic circulation. The first one, inserted according to a technique previously described (Proc. Soc. Exp. Biol. \& Med., 74 : 551, 1950), recorded the output of the left ventricle with the exception of the amount of blood flowing through the arteriovenous fistula which was recorded by the second rotameter. The magnitude of the fistula established between the root of the aorta and the proximal end of one of the femoral veins could be adjusted by a tunnel-clamp. Mean arterial blood pressure was recorded simultaneously with the amount of blood flowing through the fistula and the "body flow," i.e. the output of the left ventricle minus the fistula flow. The fistulas were established progressively, maintained for five minutes, then suddenly occluded. The results observed can be summarized as follows:

With "small" fistulas, i.e. fistulas accommodating a blood flow of up to 20 per cent of the control cardiac output, generally neither the "body flow" nor the mean arterial blood pressure was modified and the cardiac output was increased by an amount equal to the fistula flow. With "large" fistulas, i.e. fistulas accommodating blood flow up to around 60 per cent of the original cardiac output, the increase in cardiac output which occurred was greater than with the "small" fistulas, but the "body flow" fell below its control level; as shown by an increase in the total peripheral resistance, vasoconstriction occurred and the mean arterial blood pressure fell only slightly. With "very large" fistulas, i.e. amounting up to around 90 per cent of the control cardiac output, the increase in cardiac output was still greater; the "body flow" decreased markedly and, despite a marked vasoconstriction, an important decrease in mean arterial blood pressure occurred.

Effect of Desoxypyridoxine on Acute Lymphatic Leukemia of Adults. David R. WeIR, Cleveland, Ohio. (Introduced by Joseph T. Wearn).

Desoxypyridoxine, a potent antagonist of pyridoxine, is capable of producing pyridoxine deficiency in animals and 
man. Necessity for a pyridoxine-deficient diet can be eliminated by administration of larger amounts of desoxypyridoxine. Thus, desoxypyridoxine administration to mice ingesting non-deficient diets results in the characteristic hematologic changes of granulocytosis, lymphopenia, and myeloid metaplasia in the spleen and these changes can be prevented or reversed by pyridoxine administration. Reversal of the granulocyte: lymphocyte ratio occurs in other conditions, including cortisone administration. The pyridoxine deficiency changes have been induced in adrenalectomized mice, however, indicating that they are not mediated through the adrenal glands.

In mice having a mixed type of transplanted leukemia, pyridoxine deficiency increased the severity of the disease with excess granulocyte production and decreased survival time.

Five adult patients having acute lymphatic leukemia were maintained on a normal diet and given desoxypyridoxine in divided oral doses of up to $1.4 \mathrm{gm}$. daily for 80 days. Toxic effects were not observed nor did seborrheic dermatitis appear. In one patient the leukocytes decreased from 90,000 to 8,000 per cmm. with almost complete disappearance of abnormal cells from the blood and with a rise in hemoglobin. Marked regression of enlarged lymph nodes and spleen occurred. In a second patient the leukocytes decreased from 120,000 to 7,600 per $\mathrm{cmm}$. and moderate regression of nodes occurred. In a third patient there was regression of nodes and improvement of clinical condition without significant hematologic change. Two other patients who were in extremis died shortly after the institution of treatment.

Thus, pyridoxine deficiency is associated with specific hematologic changes. Preliminary trial suggests that induced pyridoxine deficiency may be of some use in the treatment of certain human acute leukemias but its effectiveness requires further evaluation.

\section{Augmentation by Cortisone of Nitrogen Mustard-induced Leukopenia. Austin S. WeIsBerger and BenNetT Levine, Cleveland, Ohio. (Introduced by R. F. Parker).}

Cortisone and ACTH are therapeutically effective in certain conditions in which nitrogen mustard $\left(\mathrm{HN}_{2}\right)$ is beneficial. Because granulocytosis occurs following administration of the hormones, it has been suggested that they might be capable of alleviating the leukopenia induced by $\mathrm{HN}_{2}$. However, in investigating the role of the spleen in $\mathrm{HN}_{s}$-induced leukopenia, it was observed that $\mathrm{HN}_{2}$-treated rabbits in which sham operation with simple abdominal incision was performed developed greater leukopenia than unoperated controls. This indicated that the leukopenia was greater under conditions of stress.

Administration to rabbits of $10 \mathrm{mg}$. of cortisone per $\mathrm{Kg}$. body weight for 2 days prior to, or $5 \mathrm{mg}$. per $\mathrm{Kg}$. for 3 days after, the injection of $\mathrm{HN}_{2}$ did not prevent the characteristic severe leukopenia but resulted instead in a significant increase in the degree of leukopenia and neutropenia. In animals receiving both $\mathrm{HN}_{2}$ and cortisone the neutrophils decreased to $3 \%$ of the initial value, while in animals given only $\mathrm{HN}_{2}$ there was a decrease to $22 \%$ of the initial value. Similarly, cortisone administration lessened the granulocyte protective effect obtained when L-cysteine is administered just prior to injection of $\mathrm{HN}_{2}$.

The mechanism by which cortisone administration and stress augment the severity of the $\mathrm{HN}_{2}$-induced leukopenia is not known. We have proposed that the protection against this leukopenia afforded by the prior administration of L-cysteine and closely related compounds results from competition by these compounds with $\mathrm{HN}_{2}$ for some substance necessary for normal leukopoiesis. Cortisone has been shown to inactivate $\mathrm{SH}$-containing compounds so that the increased severity of the leukopenia produced by administration of both cortisone and $\mathrm{HN}_{2}$ may be the result of cumulative interference with the metabolism of certain substances which normally contain or combine with $\mathrm{SH}$-containing compounds and which are necessary for normal leukopoiesis.

The Site of the Saluretic Action of an Organic Mercurial Compound. L. G. Welt, A. V. N. Goodyer, J. H. Darragh, W. A. Abele and W. H. Meroney, New Haven, Conn. (Introduced by John P. Peters).

The current concept of renal physiology implies that proximal tubular reabsorption of solutes is active and that the reabsorption of water follows as a passive consequence. The extent of proximal reabsorption of solute determines the volume of fluid, isosmotic with glomerular filtrate, delivered to the distal tubule, where reabsorption of water is active and largely controlled by antidiuretic hormone. With ordinary volumes of distal tubular fluid, maximum reabsorption of water is limited by a total solute concentration approximating 1,400 mOsm./L. However, when the volume of fluid delivered to the distal tubule is excessive, the final urine may be dilute despite continuous maximal antidiuretic activity.

Thus, a decrease in proximal tubular reabsorption of salt would be expected to promote the excretion of large volumes of dilute urine despite maximal antidiuretic activity. A decrease in distal reabsorption of salt, however, would promote only a modest increase in volume of a urine with persistently high total solute concentration.

Three normal hydropenic male subjects received a continuous infusion of saline containing sufficient pitressin to insure the administration of 100 milliunits an hour. When a steady state had been achieved, 2 cc. of thiomerin were administered. This promoted an increase in the rates of excretion of water, sodium, and chloride, little or no change in ammonia and urea, a decrease in potassium, and no essential change in the clearance of endogenous creatinine. The augmented rate of excretion of salt was accompanied by a decrease in the concentration of each of the measured urinary solutes including sodium and chloride.

These studies imply that the influence of mercury with respect to the tubular reabsorption of salt must be proximal, although it may be distal as well. 
Coagulation Changes in the Isolated Venous Segment of the Dog. Stanford Wessler, Boston, Mass. (Introduced by Benjamin Alexander).

The mechanisms involved in the initiation and propagation of intravascular clotting remain obscure. Studies to elucidate certain aspects of this problem have been carried out in the dog. Isolated blood-filled venous segments were prepared in 35 animals so that the blood was completely stagnant and the vascular segment entirely cut off from the general circulation. In these segments containing 1-2 cc. of blood, grossly visible clots rarely appeared within 60 minutes but always formed within 120 minutes. In six dogs the prothrombin concentration of the blood in these segments was determined two minutes to nine hours after isolation of the segment. By the two-stage method, prothrombin concentration remained unchanged for four hours despite evident deposition of a clot. Thereafter, prothrombin decreased slowly, but did not disappear completely in nine hours. The slow rate of platelet disappearance and the one-stage prothrombin values, which paralleled the two-stage results, confirmed the marked deceleration of clotting afforded by endothelial tissue.

In contrast, dog serum and purified SPCA (an accessory prothrombin conversion factor in serum) uniformly produced a clot within three minutes after their injection into the segment. This is the first time that SPCA, which evolves as a consequence of clotting, has been shown to act as a clot-promoting agent in an endotheliallined segment. Clots produced in this manner do not adhere to the endothelial wall during the first 48 hours after their formation and therefore act as emboli when the continuity of the venous segments with the general circulation is restored.

The use of this approach may facilitate study of the earliest phases of the coagulation sequence and should permit study of clot propagation and clot adherence under a variety of altered physiologic states.

\section{Production of Water Retention and Acute Hyponatremia Without Sodium Loss by Administration of Pitressin Tannate to Patients in Congestive Heart Failure. $\mathrm{R}$. E. Weston, I. B. Hanenson, E. R. Borun, J. Gross- MAN and M. Wolfman, New York, N. Y. (Intro- duced by Louis Leiter).}

Previous studies have indicated that intensification of congestive failure by severe respiratory infection or inadequate digitalization may lead to water retention and acute hyponatremia without sodium loss in cardiac patients on low salt diets. To evaluate the role of increased $\mathrm{ADH}$ activity in this phenomenon, the effect of daily intramuscular injections of Pitressin Tannate in Oil (PTO) on metabolic balances of water, sodium, potassium, chloride, nitrogen and phosphorus, corresponding blood chemistries, and eosinophile counts of seven rheumatic cardiacs in chronic congestive failure was studied.

On 5 units of PTO daily for 3 to 7 days, four cardiacs, maintained on $2000-2700 \mathrm{ml}$. fluid and $15 \mathrm{~m}$.eq. sodium intakes, had reduced urine flows, 1.8 to $4.9 \mathrm{kgm}$. weight gains, and 17-27 m.eq./1. decreases in serum sodium and chloride concentrations. Concurrently, they developed increased edema, dyspnea and venous pressure, eosinopenia, and further reduction in urinary sodium output. When restudied on higher salt intakes, two of these patients exhibited greater electrolyte retention and, consequently, smaller decreases in serum sodium and chloride levels during PTO administration. Within 24-48 hours after discontinuing PTO, water diuresis began. In 24-96 hours, pre-pitressin status was restored, and urinary sodium and chloride outputs increased to above levels.

Three cardiacs manifested no significant changes on 5 units of PTO daily. This dose, twice daily, promptly produced the response described above in two of these patients. Despite continued administration of PTO, after 16 days one of these had spontaneous water diuresis accompanied subsequently by natriuresis.

In one non-cardiac hyperthyroid patient on a full salt intake, 5-15 units of PTO daily for 9 days produced no changes.

Calculated fluid and electrolyte shifts, and the significance of water retention in the pathogenesis of acute hyponatremia in congestive failure, will be discussed.

\section{Calcium Loss in Paralytic Poliomyelitis and Its Quanti- tative Relationship to the Development of Demonstrable Osteoporosis. G. Donald Whedon, New York City,} N. Y. (Introduced by Ephraim Shorr).

Metabolic balance studies were carried out for 6 to 10 months in 9 patients with acute anterior poliomyelitis ranging in age from 17 to 39 years. Paralysis was generally marked and extensive. Studies on 4 of the patients were begun early in the acute phase. As soon as possible constant diets were instituted, the calcium intake being $920 \mathrm{mgm}$. daily. Standard methods of therapy were used.

Progressive increase in both urinary and fecal calcium was noted. The maximum urinary calcium (for a sixday metabolic period) ranged from 391 to $789 \mathrm{mgm}$./day, the mean being $572 \mathrm{mgm}$./day, and occurred on the average 5 weeks after onset. Urinary calcium began to decline slowly before ambulation was begun but remained above $250 \mathrm{mgm}$./day for $5 \frac{1}{2}$ months. Excellent background for urinary tract calculus formation was afforded by extensive increases in urinary phosphorus as well as calcium, accompanied by little change in urinary volume and citric acid.

The mean maximal negative calcium balance $(-0.540$ gm./day) occurred during the ninth week. Mean duration of negative balance was 7 months, the patients having been recumbent for 6 months on the average.

Mean serum calcium during the first four months was $10.8 \mathrm{mgm} . \%$; in two patients, however, one-third of the values were above $11.5 \mathrm{mgm} . \%$.

On independent evaluation of monthly $x$-rays by a radiologist unaware of the metabolic data, initial evidence of osteoporosis, usually patchy demineralization and coarsening of trabecular markings in the lower extremities regional to joints and most often in necks and distal ends of the femora, appeared approximately three months after onset with average loss of $2 \%$ of estimated total 
body calcium. Total calcium losses for the 7 months of negative balance averaged $4 \%$ and ranged up to $8.5 \%$ without detectable $\mathbf{x}$-ray changes in the spine.

The Effect of Intravenous Pitressin on the Renal Excretion of Water and Electrolytes in Congestive Heart Failure and in Liver Cirrhosis. Abranam G. White and George Rubin, New York, N. Y. (Introduced by Ernst P. Boas).

An infusion of $5 \%$ glucose in water was administered intravenously at $10 \mathrm{cc}$. per minute to seven control subjects, ten cardiacs, and five patients with liver disease (four with decompensated liver cirrhosis and one with infectious hepatitis), and the resulting water diuresis studied. While the glucose infusion continued and after the urine flow reached a plateau, $0.57 \mathrm{mU} / \mathrm{kg}$ of Pitressin was injected intravenously into the seven controls, into six of the cardiac patients and into the five hepatic patients.

Following onset of the infusion the average time required for attainment of peak diuresis in the control subjects, cardiac patients, and liver patients was: $85.1 \pm$ $32.3,99.1 \pm 32.1$, and $88.6 \pm 27.2$ minutes, respectively. The average peak urine flows attained during the hydration periods were: $12.0 \pm 2.7,6.7 \pm 3.3$, and $11.9 \pm 3.0$ $\mathrm{cc} /$ minute for the three groups.

There was no significant difference in the duration of, and intensity of, Pitressin antidiuresis under these experimental conditions in three groups of patients, except for two of the cardiacs who demonstrated an enhanced water antidiuresis. In the dosage administered, Pitressin did not exert any significant effect on the urinary output of sodium, chloride or potassium. As for the effect of Pitressin on the concentrations of electrolytes in the serum, the only consistent change noted was that of the serum sodium which tended to fall during Pitressin antidiuresis in the five patients with liver disease. The two cardiac patients who showed the augmented Pitressin effect also had the lowest concentrations of serum sodium (121.9 and $130 \mathrm{mEq} / \mathrm{L}$ ), but a causal relationship remains to be established.

In summary, these data offer no conclusive evidence for any deficiency in the mechanisms of inactivation of Pitressin administered in physiological dosage to controls, cardiacs or patients with liver disease.

PTC Factor: A New Plasma Thromboplastin Component Distinct from the Anti-Hemophilic Factor. SIDNEY G. White, Paul M. Aggeler,* Mary Beth Glendening, Ernest Page. Tillie B. Leake and George Bates, San Francisco, California.

A 16-year-old male was found to have normal amounts of all known coagulation factors, no increase in circulating anticoagulants, markedly prolonged whole blood coagulation time and defective prothrombin utilization. The defect was corrected by accelerator-free tissue thromboplastin and by "platelet-free" normal and hemophilic plasma, but not by antihemophilic globulin (Cohn's Fraction I) (AHG). These findings indicate the ab- sence from his plasma of a previously undescribed coagulation factor, the plasma thromboplastin component (PTC), which is biologically as important as AHG in the production of thromboplastin in blood.

PTC is adsorbed from oxalated plasma by all known prothrombin adsorbants and by Seitz filtration. It is associated with the plasma euglobulins, and unlike AHG is present in the serum. It is most stable in the neutral ranges and is heat stable. It can be separated from previously described coagulation factors and concentrated as follows: Oxalated "platelet-free" plasma is heated to $56^{\circ} \mathrm{C}$. for 5 minutes to destroy prothrombin, spca, Factor $\mathrm{V}$, and labile factor. The coagulated fibrinogen is removed by centrifugation. PTC is separated from AHG by adsorbing it on $\mathrm{BaSO}_{4}$, from which it is eluted with $5.8 \%$ sodium citrate. The thrombin-free eluate is dialyzed against $0.9 \% \mathrm{NaCl}$ and lyophylized.

The patient's PTC fraction assayed against his own blood, using a "two-stage" prothrombin utilization test, is inert, while that obtained from normal, hemophilic, thrombocytopenic, and dicumarolized hypoprothrombinemic plasma is significantly active.

PTC is not a platelet factor, AHG, or a "complete" thromboplastin, such as that derived from tissue sources, since it will not correct the defect in "platelet-free" normal plasma or platelet-rich hemophilic plasma. It is not the plasma thromboplastin of Chargaff and West, since the supernatant from normal plasma centrifuged at 20,000 r.p.m. for 150 minutes completely corrects the patient's defect.

Hemodynamic Changes in Patients with the Eisenmenger Complex. K. Willis, T. J. Reeves, E. Booth, T. A. Lombardo, C. Lombardo and S. TUluY, Birmingham, Alabama. (Introduced by T. R. Harrison).

Findings by means of cardiac catheterization and electrokymographic studies of the same patients have been as follows:

(1) The interventricular shunt is indicated by characteristic reciprocal paradoxic movement of the ventricular borders. These indicate a left-to-right shunt (mainly in systole) and a right-to-left shunt (mainly in diastole).

(2) Paradoxic border movement due to volume changes produced by intraventricular shunts must be distinguished from paradoxic movement due to shape changes which are a normal phenomenon. Criteria for such distinction are presented.

(3) The experience to date points toward distinctive electrokymographic records in patients with the Eisenmenger complex. However, it is as yet uncertain whether similar border movements are present in other types of congenital malformation associated with interventricular septal defects.

Dynamic Response Characteristics of Manometer Systems Necessary for Adequate Recording of Intravascular Pressure Pulses in Man. EARL H. Wood,* Rochester, Minnesota.

Practical considerations necessitate the use of small needles or long narrow bore plastic tubes for direct 
recording of pressures in the vascular system of man. Minimum cross-sectional dimensions and maximal length of needles or tubes suitable for this purpose are governed by the dynamic response characteristics of manometer systems necessary for recording of the dynamic components of practical importance in the pulse wave. There are, however, no direct data in the literature concerning just how much the dynamic response of manometer systems can be reduced without producing significant distortion of recorded pulse waves.

Intrabrachial arterial pressure was photographed continuously while intraradial pressure was simultaneously recorded from the same arm with 5 different manometer systems of widely different dynamic response. Differences in radial systolic pressure and pulse contour could not be detected in 11 normal and 5 hypertensive patients when manometer systems with uniform response out to 6 cps and above were used. However, a decrease in radial systolic pressure averaging $4 \mathrm{~mm}$. $\mathrm{Hg}$ ( $P$ value 0.01 ) was detected in recordings from a manometer system with uniform response to 2 and a 90 per cent response at 4 cps. None of the manometers changed diastolic or mean pressure.

Similar results were obtained in studies of aortic pressure pulses using Peterson catheters attached interchangeably to capacitance and strain gauge manometers and in studies of intracardiac pressures by means of catheters tipped with miniature manometers.

It is concluded that dynamically adequate manometer systems can be assembled even when miniature $(1 \mathrm{~mm}$. O.D.) 1 meter length catheters are used. Amplifier instability and artefacts due to rhythmic catheter motion are much more frequent sources of non-recognized errors in pressure recordings via catheters than are the amplitude and phase distortion characteristics of the manometer systems used.

The Normal and Modified Erythrocyte: Observations of Certain Phagocytable Characteristics. Claude-StarR Wright, Matthew C. Dodd, Joseph A. Bass and Noreen G. Brandt, Columbus, Ohio. (Introduced by Charles A. Doan).

There has been little correlation between in vivo and in vitro tests for qualitative abnormalities in the erythrocytes. New in vitro means have therefore been sought to determine the earliest minimal changes in erythrocyte integrity. This has been partially accomplished by the determination of phagocytic indices utilizing normal and modified erythrocytes as test cells. Macrophages cultured from rabbit spleens in the presence of homologous serum were the stock phagocytizing cell. Normal and modified erythrocytes were added to tissue cultures of 3-5 day old macrophages. Species differences between the rabbit and the human were not apparent since normal erythrocytes from both were phagocytized by only $0-10 \%$ of the rabbit macrophages in the control cultures. Cells from healthy adult humans were used to establish these base lines.

Studies to date indicate that the following modified erythrocytes are more easily phagocytized than are nor- mal cells : (1) trypsinized RBC, (2) sensitized RBC, (3) RBC from patients with hereditary spherocytosis and acquired hemolytic anemia. Phagocytosis of erythrocytes from rabbits receiving 700 $\mathrm{r}$ total body irradiation and tested over 27 days after irradiation was not increased over the normal.

Treatment of Pemphigus with Potassium Para-Aminobenzoate. Chris J. D. Zarafonetis and John Shaw, Philadelphia, Pennsylvania. (Introduced by Arthur C. Curtis).

Prolonged administration of large doses of potassium para-aminobenzoate (KPAB) has been shown to be beneficial in a variety of clinical disorders. Favorable results in dermatitis herpetiformis suggested a trial of KPAB therapy in another bullous condition, namely, pemphigus. The purpose of this report is to present briefly the results achieved with KPAB in the treatment of 15 patients with various types of pemphigus. Included are 7 patients with pemphigus vulgaris, 5 with pemphigus foliaceous, 2 with pemphigus erythematodes, and 1 with ocular pemphigus.

KPAB was administered as a $10 \%$ solution, usually 30 cc. $(3.0 \mathrm{gm}$.) every 3 hours for 6 to 8 doses daily; i.e., a total of 18 to $24 \mathrm{gm}$. per day. Twelve patients had improvement characterized by cessation of exudation, subsidence of blister formation, followed by epithelialization and hyperpigmentation. Plasma specimens from several patients were subjected to electrophoretic analysis by Dr. Marie Berg, who noted general improvement in the protein pattern during the period of KPAB treatment. Those patients whose disease was long-standing responded more slowly than those with relatively recent onset. The duration of therapy varied, therefore, from a few weeks to several months. In those cases where complete suppression was achieved, treatment was discontinued and the patients observed for relapse. Remissions were maintained for as long as 7 months. Two patients who had good, though incomplete, suppression on KPAB were treated with cortisone without added improvement. It is to be emphasized that KPAB is not curative. Nevertheless, its use in the management of selected cases of pemphigus appears warranted.

\section{Hemodynamic Studies by the Opacification Technique in} Patients with Mitral Stenosis and Other Cardiovascular Abnormalities. Harry F. Zinsser, Paul W. Eyler, David P. McCallie and William J. Tuddenham, Philadelphia, Pennsylvania. (Introduced by Calvin F. Kay).

In the angiocardiographic technique, individual cardiac chambers and great vessels become opacified as seen on $x$-ray films taken at rapid intervals following the intravenous injection of contrast material. The changes in density of a given chamber from film to film can be measured photoelectrically by a densitometer. When these density measurements are plotted against time, opacification curves can be constructed for individual chambers, and from numerous curves, a mean opacity curve can be 
derived. Such curves are related to the velocity of blood flow and to the various factors governing it.

The mean right heart curve for normal subjects showed a rapid rise and slower fall. The entire curve was completed by six seconds. This curve was altered in various conditions which delay emptying of the right heart, for example in cor pulmonale, Ebstein's anomaly, and pulmonic stenosis.

In normal subjects, opacification of the left heart chambers and aorta showed an essentially uniform duration and intensity. The mean left heart curve for normal subjects began at five seconds and faded by ten seconds after injection.

In mitral stenosis, right heart density curves were not significantly altered except where secondary pulmonary vascular changes were proven or suspected. Left heart curves, however, were markedly altered. The left atrium remained sharply demarcated for long periods of time. The beginning of its mean curve was not significantly different from that of normal subjects. However, the remainder of the curve rose to high levels of opacity and did not fade until 26 seconds after the injection of opaque material. The left ventricle and aorta were poorly opacified and their mean curves differed sharply from the atrial curve. Such characteristic alterations in the duration and degree of opacification of individual heart chambers provide information of value in the selection of patients for mitral commissurotomy.

The Effect of Drugs and Anemia upon Experimentally Produced Interarterial Coronary Anastomoses. PAUL M. Zoll and Leona R. Norman, Boston, Mass. (Introduced by Mark D. Altschule).

The time required for the development of interarterial coronary anastomoses and the effects of drugs and anemia on this process were studied in 349 young domestic pigs. Coronary anastomoses of functional significance are initially absent in this animal. Acute complete ligation of the right coronary artery in 24 pigs resulted uniformly in death. Injection of the coronary arteries with colored lead-agar mass by the Schlesinger technic in these acute experiments and in 134 normal, control pigs showed uniform absence of extensive anastomoses.

Functionally significant anastomoses were demonstrated, however, following partial narrowing of the right coronary artery to 15 to 35 per cent of its original area. The same artery was subsequently occluded completely at a second operation. Functionally significant anastomoses were demonstrated by survival following final occlusion or by extensive retrograde injection of vessels distal to the occlusion. A significant effect of narrowing in stimulating anastomoses was first apparent after two days. The frequency of anastomoses increased with longer intervals, but they were not very frequent till after 12 days or more. Various vasomotor drugs were administered in the intervals between narrowing and occlusion; sodium nitrite alone increased the development of anastomoses significantly.

Thirty-nine animals were made anemic or were given ,vasomotor drugs for 1 to 6 weeks without preliminary coronary artery narrowing. Extensive anastomoses were found in 1 of 4 animals treated with sodium nitrite and in 2 of 14 anemic pigs. These few positive results are statistically significant in comparison with the absence of such anastomoses in 134 control hearts.

These studies delineate the rate of development of anastomoses after coronary artery narrowing. They also indicate that sodium nitrite accelerates their development and they confirm experimentally the efficacy of anemia in stimulating anastomoses that was suggested by our previous clinico-pathological studies in man. 